$$
\begin{array}{r}
M 230860 \\
\text { CR } 1239 \%
\end{array}
$$

\title{
SYNTHESIS OF SHUTTLE VEHICLE DAMPING USING SUBSTRUCTURE TEST RESULTS
}

\author{
by
}

Daniel D. Kana Stephen Huzar

INTERIM REPORT

$\rightarrow \mathrm{m}$ Contract No. NAS8-27569

Control No. 1-1.50-13733 (2F)

SwRI Project No. 02-3131

\section{Prepared for}

National Aeronautics and Space Administration George C. Marshall Space Flight Center Marshall Space Flight Center, Alabama

June 1972

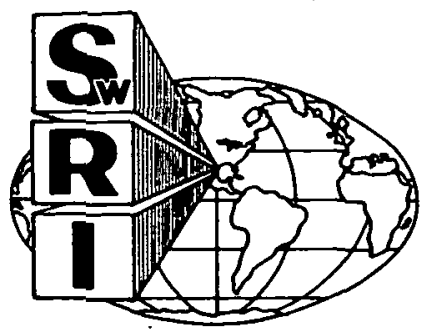


SOUTHWEST RESEARCH INSTITUTE

Post Office Drawer 28510, 8500 Culebra Road

San Antonio, Texas 78284

\title{
SYNTHESIS OF SHUTTLE VEHICLE DAMPING USING SUBSTRUCTURE TEST RESULTS
}

by

Daniel D. Kana

Stephen Huzar

\author{
INTERIM REPORT \\ Contract No. NAS8-27569 \\ Control No. 1-1-50-13733 (2F) \\ SwRI Project No. 02-3131
}

Prepared for

National Aeronautics and Space Administration

George C. Marshall Space Flight Center

Marshall Space Flight Center, Alabama

June 1972

Approved:

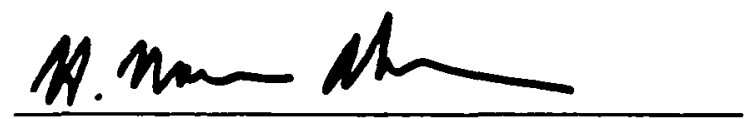

H. Norman Abramson, Director

Department of Mechanical Sciences 


\begin{abstract}
An empirical method is developed for predicting the modal damping of a combined parallel-stage Shuttle model by means of damping measurements performed on the individual substructures. Correlations are first determined for each component in terms of damping energy as a function of peak kinetic energy and modal amplitude. The results are then used to predict component damping energies corresponding to the respective kinetic energies and amplitudes that occur for the new modes of the combined System. Modal characteristics for the System, other than damping, are obtained by a real eigenvalue solution of dynamic equations developed by Hurty's procedure of substructures. System equations, which include component modal damping, are also solved by a complex eigenvalue approach for comparison with results of the empirical method.

The experimental model consists of two beam-like substructures, each of which contains a series of discrete rigid masses, and can be joined into a single system through three pin-joints. Variable damping is provided by means of multiple paddles acting in oil reservoirs. Joint damping is also provided by means of a magnetic loop which is modulated by an active feedback circuit. Components are tested in pin-slip and free-free support conditions, and the System is tested in the free-free condition. A variety of damping and mass configurations are included. The empirical method is found to provide damping predictions within $10 \%$ to $20 \%$ error, while the complex eigenvalue results deviate by as much as $300 \%$ ! Recommendations are given for application of the empirical method to a four-component, more representative Shuttle configuration.
\end{abstract}


LIST OF ILLUSTRATIONS iv

LIST OF TABLES vi

PRINCIPAL NOTATION vii

I. INTRODUCTION 1

II. DESCRIPTION OF PHYSICAL MODEL 3

A. Configurations 3

B. Damping Mechanisms 8

III. DEVELOPMENT OF ANAI،YTICAL MODELS 10

A. Coordinate System 10

B. Component Eigenvalue Problems 16

C. Component Forced Response 24

D. System Equations 27

IV. EXPERIMENTAL PROCEDURES 40

A. Background Discussion 40

B. General Procedures 43

C. Link Damper Calibration 44

V. COMPONENT RESULTS 46

A. Pin-Slip Configuration 46

B. Free-Free Configuration 55

VI. SYNTHESIS OF SYSTEM DAMPING 66

$\begin{array}{ll}\text { VII. SYSTEM RESULTS } & 68\end{array}$

A. Natural Modes 68

B. Damping 74

C. Forced Response $\quad 78$

D. Verification of Damping Synthesis Hypothesis 85

$\begin{array}{ll}\text { VIII. FINAL DISCUSSION } & 89\end{array}$

REFERENCES $\quad 92$

ACKNOWLEDGEMENTS . 93

APPENDIX A - Values for Elements of Discrete Models A-1

APPENDIX B - Derivation of Element Stiffness Matrix B-1 
1. Conceptual Design of Space Shuttle Model 4

2. Photograph of Space Shuttle Apparatus 7

3. Link Damping Mechanism 9

4. Coordinate System 11

5. Influence of Peak Kinetic Energy and Amplitude on Damping Energy for Booster Model 42

6. Booster Mode Shapes for Pin-Slip Configurations

a. Ml Mass Condition 47

b. M2 Mass Condition 48

7. Orbiter Mode Shapes for Pin-Slip Configurations
a. M1 Mass Condition
b. M2 Mass Condition

8. Experimental Damping Energy for Pin-Slip Component Configurations

$\begin{array}{ll}\text { a. Booster } & 53 \\ \text { b. Orbiter } & 54\end{array}$

9a. Booster Component Response at Mass 10 for B-P-L1-D2-M1 Configuration

9b. Orbiter Component Response at Mass 4 for O-P-L1-D2-M1 Configuration

10. Booster Mode Shapes for Free-Free Configurations Ml Mass Condition

11. Orbiter Mode Shapes for Free-Free Configurations M1 Mass Condition

12. Experimental Damping Energy for Free-Free Component Configurations
a. Booster
b. Orbiter 


\section{LIST OF ILLUSTRATIONS (Cont'd.)}

Figure No.

$\underline{\text { Page }}$

13a. Booster Component Response at Mass 10 for

B-F-L0-D2-M1 Configuration

13b. Orbiter Component Response at Mass 4 for O-F-L0-D2-M1 Configuration

14. Combined System Mode Shapes

a. Ml Mass Condition

$69-70$

b. M2 Mass Condition

$71-72$

15. Combined System Damping Energy

16. System Response for S-F-L1-D2-M1 Configuration
a. Booster Mass 10
b. Orbiter Mass 4

17. System Response for S-F-L1-D3-M1 Configuration
a. Booster Mass 10
b. Orbiter Mass 4

18. System Response for S-F-L3-D2-M1 Configuration
a. Booster Mass 10
84
b. Orbiter Mass 4

19. Orbiter Damping Energy at Arbitrary Frequencies

20. Damping Energy for SAD-6 Booster Vehicle for Fixed Modal Amplitude 
I(a) Test Configurations

I(b) Configuration Nomenclature

II. Comparison of Natural Frequencies Obtained by Direct and Transformation Methods

III. Link Damping Coefficients for L2, L3, L4

IV. Component Natural Frequencies and Modal Mass for Pin-Slip Support
(a) Booster
(b) Orbiter

V. Component Damping for Pin-Slip Support

VI. Component Natural Frequencies and Modal Mass for Free-Free Condition

VII. Component Damping for Free-Free Condition

VIII. System Natural Frequencies

(a) Pin-Slip Component Mode

(b) Free-Free Component Mode

IX.

System Damping

$\mathrm{X}$.

System Damping Including Joint Dissipation
(a) L2 Case
(b) L3 Case
(c) L4 Case

80 
PRINCIPAL NOTATION

A

[a]

[ $\beta$ ]

[C]

G

[Y]

$D_{c}$

$\delta$

$\mathrm{E}$

$\{\mathscr{F}\}$

f

$\zeta$

I, $d$

[I]

$[\mathrm{K}],[\mathrm{k}]$

[K]

$\ell$

[M ], [m]

[m]

$\mathrm{N}$

$v$

\{P\}

$\{\mathrm{p}\}$

$\{Q\}$

$\{\mathrm{q}\}$

$\mathrm{R}$

$\mathrm{T}$

t
Area

Coordinate transformation matrix

System coupling matrix

Damping matrix

Shear modulus

Eigenvector matrix

Damping energy dissipated per cycle

Link length, etc. (see Figure 4)

Modulus of elasticity

Column vector of generalized forces

Frequency $(\mathrm{Hz})$

Critical damping ratio

Moment of inertia

Identity matrix

Stiffness matrices

Generalized stiffness matrix

Element length or reference length

Mass matrices

Generalized mass matrix

Denotes normal mode elastic quantities

Poisson's ratio

Column vector of nodal forces

Column vector of generalized coordinates

Column vector of generalized applied forces

Column vector of displacements or generalized coordinates

Denotes radius or rigid-body quantities

Kinetic energy

Tube thickness or time 
PRINCIPAL NOTATION (Cont'd.)

$\mathrm{U}$

$\{u\}$

$[\phi],[\varphi]$

$\mathrm{X}$ 。

$\mathbf{x}, \mathbf{z}, \theta$

$\Psi$

$\Omega_{\jmath}$

$\omega$

$(-)$

$\left({ }^{\wedge}\right)$
Internal energy

Column vector of nodal displacements

Modal displacement matrices

Peak modal amplitude for steady-state sinusoidal vibration

Longitudinal, lateral, and rotational displacements, respectively, at the node points.

Phase angle

Ratio of forcing frequency to $j$-th natural frequency

Circular frequency

Denotes quantities are with respect to coordinate system where all nodes are interior nodes. Also denotes normalized quantity.

Denotes quantities are with respect to coordinate system where some nodes are on boundary and the rest are interior nodes 


\section{INTRODUCTION}

Prediction of damping in complex structures is recognized to be extremely difficult because of the presence of various damping mechanisms and the current lack of knowledge in synthesizing even the most elementary mathematical models into combined structural systems. As a result, in initial design procedures in the past, damping characteristics of aerospace systems have usually been predicted at the modal level by empirical methods based on data derived from previous vehicles $(1)^{*}$, and when possible, ultimately verified by actual measurements of modal damping in full-scale dynamic tests. Needless to say, such full-scale testing of large systems is extremely difficult and expensive at best, and has been deemed unfeasible for the Space Shuttle, which will be the largest and most complex aerospace structural system yet attempted.

In order to provide information necessary for predicting dynamic response for Space Shuttle, it is recognized that some new procedure must be developed for this multi-component system. As a reasonable approach, full-scale testing of the Booster, Orbiter, and any other major components appeared possible, but a requirement then exists for synthesizing this information into a prediction of response for the combined System. Substructuring methods are available $(2)$, and are presently being further developed(3) for handling prediction of dynamic characteristics of undamped structures. However, no accurate method has been derived for including damping in such structural synthesis procedures. Therefore, the purpose of this report is to present the development of a method which shows great promise for accomplishing this objective. The philosophy followed in the research program leading to the se results has been to perform dynamic tests on individual substructures of a two-component, parallel-stage, rather simple Space Shuttle model, develop a mathematical model for the components, combine all of the information into a mathematical model for the combined System, and compare predicted dynamic characteristics with those obtained from further tests of the combined System.

At the outset of this program it was recognized that enormous efforts have been exerted in the past $(4,5)$ to develop mathematical models of various elementary damping mechanisms, and this work will continue because of the great uncertainty present in the current state-of-the-art in combining these mechanisms in complex structures. It was also recognized

* Superscript numbers in parentheses denote references on page 92 of this report. 
that the most reliable methods currently available for measurment of typically light damping in complex structures having reasonable modal separation, are those which determine an overall modal damping at resonance or free decay from resonance conditions. The beauty of the concept of modal damping, of course, is that it results in a single number that represents a reasonable approximation to the aggregate contributions of a multitude of complex damping mechanisms internal to the oyerall structure. Once it is determined, and some form of relatively simple ${ }^{(6)}$ equivalent mathematical model is matched with the modal values of mass, stiffness, and damping, prediction of dynamic response for that component will be satisfactory, at all intermediate frequencies, since damping forces dominate only in the vicinity of resonances. Nonlinearity of damping with amplitude, and inevitable relatively wide scatter in damping measurements are two unfortunate dilemmas which must often be faced with this otherwise reasonable approach to the dynamic prediction problem.

In view of the success of modal concepts for synthesis in the past, it appeared reasonable to consider their use for Space Shuttle predictions also, although it is recognized that considerable complications result because of the combination of the substructures into an even more complex System. System natural modes can be expected to occur at frequencies which are intermediate to those for the individual components, and as a result, damping forces which were insignificant in the dynamic response at the intermediate frequencies for the individual structures, now become dominant for the new modes and frequencies. Therefore, the prediction method outlined herein, is based on determination of modal damping energy for each component from its own resonance tests, extrapolation of the contribution of each respective component to the total modal damping energy at the new frequencies, and summation of the results for all components. Results for the method will be shown to be far superior to others that were considered. Limitations will be discussed, along with additional work recommended for further verification. We begin by providing a description of the physical model used in the study, proceed with derivation of mathematical models of the System, continue with a detailed development of the damping synthesis method, and finally compare results for a multitude of tests and predictions for modal damping and dynamic response of a variety of damping and mass configurations. 


\section{DESCRIPTION OF PHYSICAL MODEL}

\section{A. Configurations}

A conceptual drawing of the Space Shuttle model is shown in Figure 1. It consists basically of Booster and Orbiter model joined together with a pin-joint near the top, and two pin-joints at the end of a rigid link near the bottom. The lower joint simulates a slip-joint for all practical purposes. Each component was made of a 2024 aluminum tube of 1 -inch $O . D$. and 0.035 -inch wall. Multiple rigid masses were positioned on each to represent the two different mass configurations indicated in Figure 1. Approximate locations of the masses can be scaled from the figure. These masses were made of split disks of $1 / 2-$ inch thick mild steel and all were nominally 6 -inch diameter and weighed approximately 3-3/4-1b each. Those masses attached to connecting joints included cutouts where necessary to accommodate the joints. These pin-joints were provided through the use of 1/4-inch diameter, Bendix Type 5008-600 flexure pivot bearings, that are designed to have extremely low inherent damping.

A list of the numerous configurations to be considered is given in Table I(a), while an explanation of the notation is given in Table I(b). It can be seen that two mass configurations were included for several different damping configurations which will be explained momentarily. Both pin-slip and free-free boundary conditions were investigated for the components in order to determine what effect this would have on predictions for the System. (This consideration would also help indicate the type of support structures required for full-scale component testing.) Pin-slip component tests were conducted by mounting the substructure directly to a rigid wall through the upper pin and lower slip-joint. Freefree component tests were conducted by hanging the substructure from a low frequency suspension which was attached to eye-bolts at each side of the second-from-the-top mass (this point was estimated to be a node for most of the lateral bending modes). Finally, System tests were conducted by having the two components attached together with a pin-joint near the top and simulated slip-joint near the bottom, while the entire System was supported on a low frequency suspension attached to a rigid knife-edge nearly under the upper pin-joint. Thus, a simulated overall free-free suspension was employed. A photograph of the model in this configuration is shown in Figure 2. 


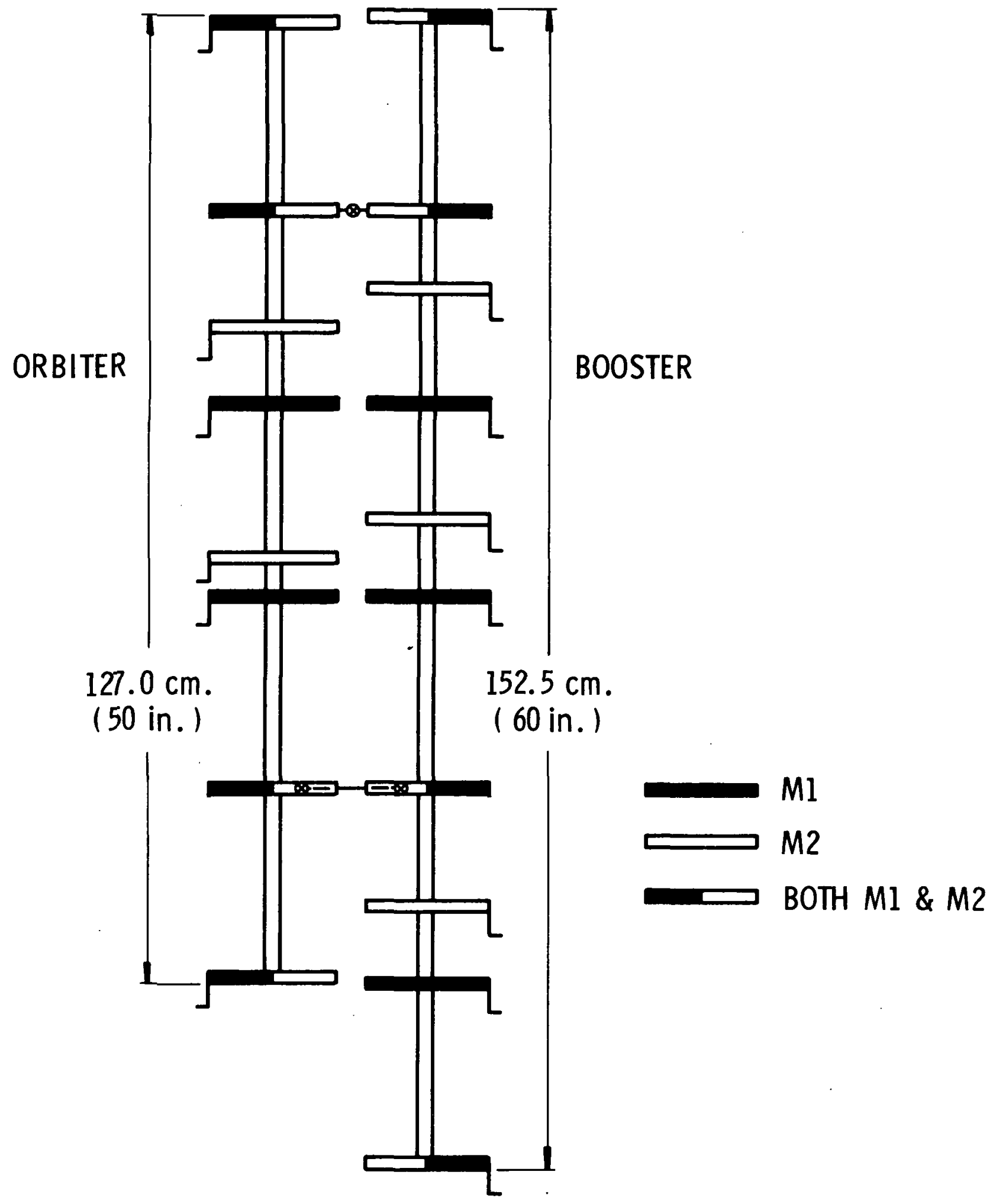

Figure 1. Conceptual Design of Space Shuttle Model 
TABLE I(a)

TEST CONFIGURA TIONS

\begin{tabular}{|c|c|}
\hline \multicolumn{2}{|l|}{ Pin-Slip Components } \\
\hline 1. $\mathrm{B}-\mathrm{P}-\mathrm{L} 1-\mathrm{D} 0-\mathrm{M} 1$ & 6. O-P-L1-D0-M1 \\
\hline 2. $\mathrm{B}-\mathrm{P}-\mathrm{L} 1-\mathrm{D} 1-\mathrm{M} 1$ & 7. O-P-Ll-Dl-M1 \\
\hline 3. $B-P-L 1-D 2-M 1$ & 8. O-P-L1-D2-M1 \\
\hline 4. $\mathrm{B}-\mathrm{P}-\mathrm{L} 1-\mathrm{D} 3-\mathrm{M} 1$ & 9. O-P-L1-D3-M1 \\
\hline 5. $B-P-L 1-D 2-M 2$ & 10. O-P-L1-D2-M2 \\
\hline \multicolumn{2}{|l|}{ Free-Free Components } \\
\hline 11. O-F-L0-D0-M1 & 16. $\mathrm{B}-\mathrm{F}-\mathrm{L} 0-\mathrm{D} 0-\mathrm{Ml}$ \\
\hline 12. $\mathrm{O}-\mathrm{F}-\mathrm{L} 0-\mathrm{D} 2-\mathrm{M} 1$ (RB Incl.) & 17. $B-F-L 0-D 2-M 1$ (RB Incl.) \\
\hline 13. O-F-LO-D3-M1 (RB Incl.) & 18. $\mathrm{B}-\mathrm{F}-\mathrm{L} 0-\mathrm{D} 3-\mathrm{M} 1$ (RB Incl.) \\
\hline 14. O-F-L0-Dl-Ml (RB Only) & 19. $\mathrm{B}-\mathrm{F}-\mathrm{L} 0-\mathrm{Dl}-\mathrm{Ml}$ (RB Only) \\
\hline 15. O-F-L0-D2-M2 (RB Only) & 20. B-F-L0-D2-M2 (RB Only) \\
\hline \multicolumn{2}{|c|}{ Additional Connecting Link Damping Included } \\
\hline 21. L1 & 23. L3 \\
\hline 22. $\quad \mathrm{L} 2$ & 24. L4 \\
\hline \multicolumn{2}{|l|}{ Combined System } \\
\hline 25. S-F-L1-D0-M1 & 30. $S-F-L 1-D 2 / D 3-M 1$ \\
\hline 26. S-F-L1-D2-M1 & 31. S-F-L2-D2/D3-M1 \\
\hline 27. S-F-L2-D2-M1 & 32. $\mathrm{S}-\mathrm{F}-\mathrm{L} 3-\mathrm{D} 2 / \mathrm{D} 3-\mathrm{Ml}$ \\
\hline 28. S-F-L3-D2-M1 & 33. S-F-L4-D2/D3-M1 \\
\hline \multicolumn{2}{|l|}{ 29. S-F-L4-D2-M1 } \\
\hline \multicolumn{2}{|c|}{ 34. S-F-L1-D3-M1 } \\
\hline \multicolumn{2}{|c|}{ 35. S-F-L1-D1-M1 } \\
\hline \multicolumn{2}{|c|}{ 36. $S-F-L 1-D 2-M 2$} \\
\hline
\end{tabular}


TABLE I(b)

CONFIGURATION NOMENCLATURE

Vehicle Identification

B - Booster

O - Orbiter

$S$ - Combined System

Support

P - Upper Support Pinned, Lower Support Slip or Double Pin

F - Components are Free-Free, System has Free-Free Support but components are joined together as with P-Support

Connecting Links

L0 - No Connection

L1 - Very Low Damping (Three Flexure Pins)

L2 - $0.5 \%$ Critical Damping for System in Air First Mode

L3 - 1.0\% Critical Damping for System in Air First Mode

L4 - 2.0\% Critical Damping for System in Air First Mode

Overall Structural Damping (Fluid Average Viscosities in $\mathrm{cp}$ )

$\begin{array}{lcc} & \text { Booster } & \text { Orbiter } \\ \text { D0 } & \text { In Air } & \text { In Air } \\ \text { D1 } & 230 & 600 \\ \text { D2 } & 1070 & 1080 \\ \text { D3 } & 4330 & 4900\end{array}$

Mass Distribution

$\left.\begin{array}{l}M 1 \\ M 2\end{array}\right\} \quad$ See Figure 1 


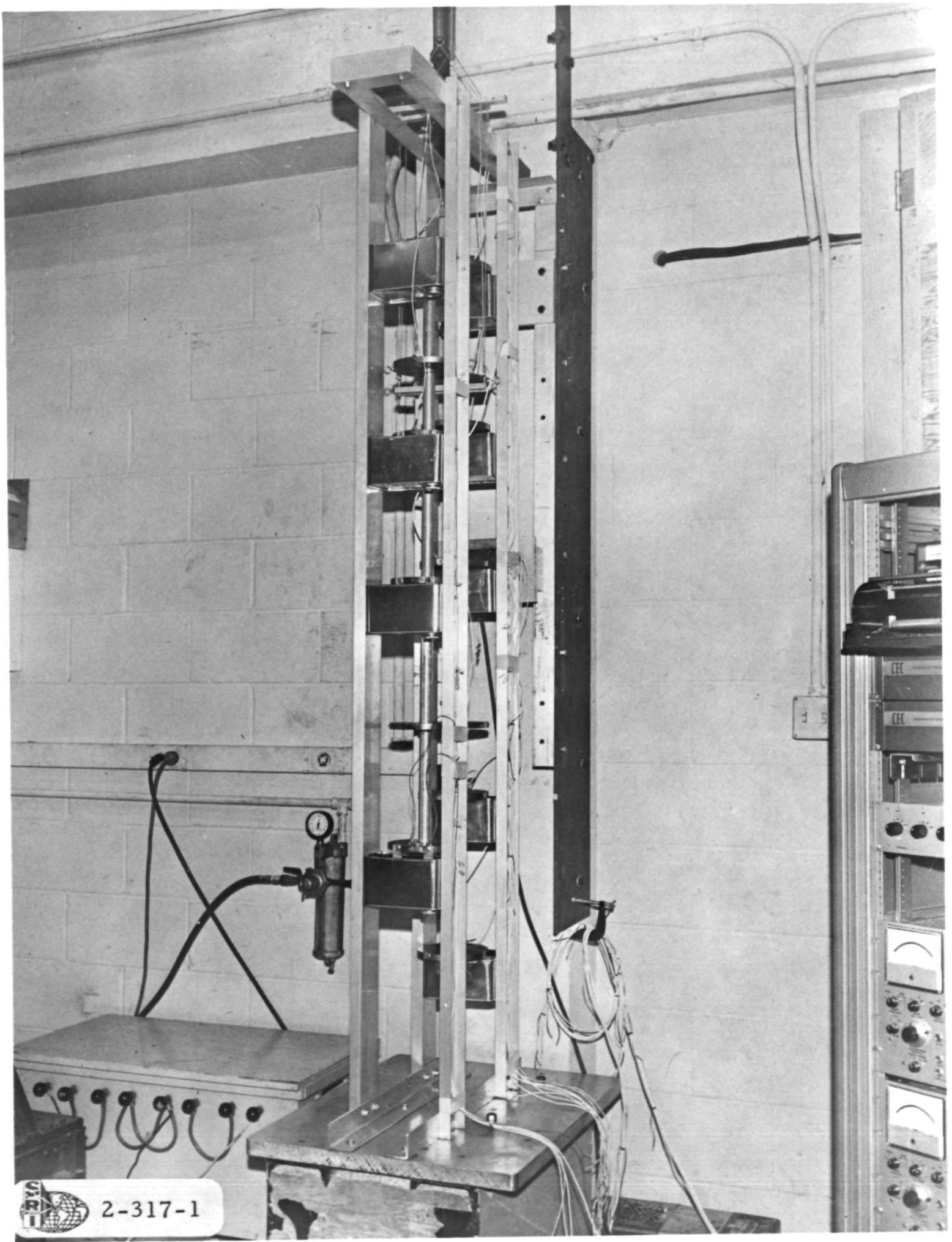

Figure 2. Photograph of Space Shuttle Apparatus 


\section{B. Damping Mechanisms}

It was desirable to use several levels of damping for the overall structure, as well as provide joint damping in the System. Since repeated tests were contemplated, it was necessary to provide damping in a form that could be controlled as accurately as possible, and be as repeatable and reliable as possible. The actual mechanism whereby damping was provided was arbitrary, so long as it had the above characteristics, and preferably provided damping forces which were linear with amplitude for the sake of increasing measurement reliability. After consideration of several mechanisms for structural damping, a series of wipers acting in oil reservoirs was selected, and the solid structure, including pin-joints, was designed to have as low inherent damping as possible.

Angle-shaped wipers were attached to the masses indicated in Figure 1. Oil reservoirs supported in their gantry-like structure can be seen in Figure 2. Thus, the model itself was suspended from the laboratory ceiling, and the dampers touched only the oil in its spacefixed reservoirs. Oil viscosity was monitored along with its temperature to allow small corrections to damping values where appropriate, as will be explained later. Average values of these viscosities are shown in Table $\mathrm{I}(\mathrm{b})$. The $\mathrm{D} 1$ and D2 damping was provided by standard motor oils, while the D3 damping fluid consisted of a mixture of heavy motor oil and STP oil additive.

Connecting link damping was provided on the Booster side of the lower slip-joint according to the approximate values given in Table I(b). Figure 3 shows the mechanism which was found to be most reliable. It consists of a permanent magnet whose flux path is split by a small gap. Part of the magnet is attached to the rigid link while the other part is attached to the connecting rigid mass of the Booster. Relative motion between the two parts of the System is measured by accelerometers $A_{1}$ and $A_{2}$, whose output is converted to relative velocity and fed back as a voltage into a coil which proportionately modulated the flux path. The low pass filter was necessary to avoid high frequency instabilities in the feedback loop. Although only one such damper was used, similar dampers could have been employed at the other two pin-joints, and the methods to be developed and results obtained would still be valid. 

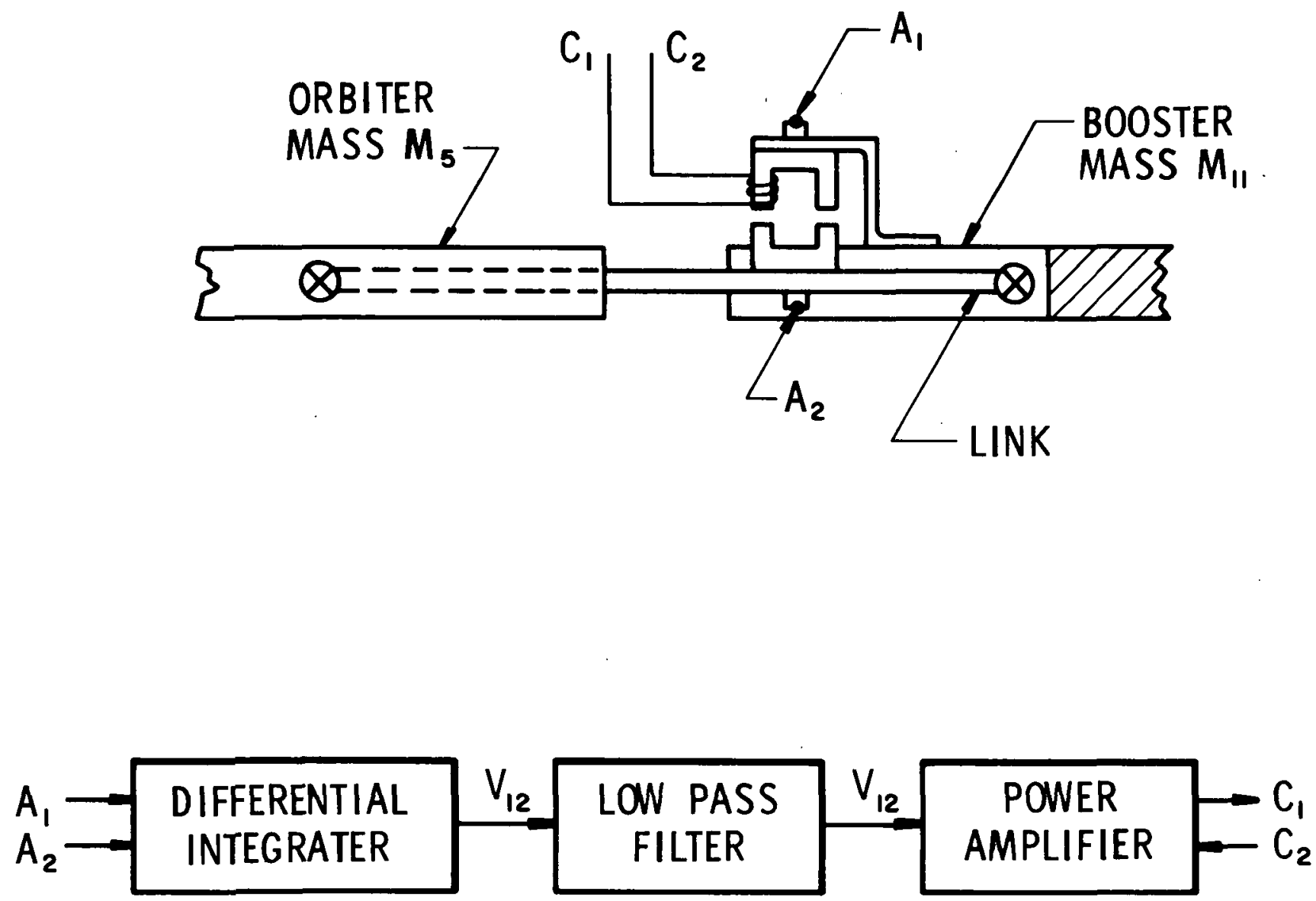

Figure 3. Link Damping Mechanism 


\section{DEVELOPMENT OF ANALYTICAL MODELS}

\section{A. Coordinate System}

The coordinate system used to describe the motion of the component Booster and component Orbiter acting as individual substructures, as well as the motion of the combined System, is shown in Figure 4. Specific values for masses, lengths, etc., are given for various configurations in Appendix A. At the $i$-th interior node point, which is located at the center-of-mass of the $i$-th concentrated mass, three planar degrees-of-freedom $\left(x_{1}, z_{1}, \theta_{1}\right)$ are permitted with the positive directions taken as indicated. Rigid body coordinates are referred to a node located at the center-of-mass of the component or System. In addition to these nodes we define a set of boundary nodes denoted by $\mathrm{Pl}$ and $\mathrm{P} 2$ at the pin-joint interface and by $\mathrm{Sl}$ and $\mathrm{S} 2$ at the slip-joint interface, respectively. Three planar degrees-of-freedom are also permitted at the se nodes, until boundary constraints are applied later. Selection of boundary nodes in this manner facilitates the solution of the component eigenvalue problem since the boundary conditions may be specified at these nodes. Likewise, when deriving the transformation matrices which couple the components together it is again convenient to specify displacement interface continuity in the appropriate directions at these nodes.

Assuming zero compliance between, say, nodes 2 and $P 1$ on the Orbiter, the relationship between the boundary displacements (node $\mathrm{Pl}$ ) and the interior displacements (node 2 ) is given by

$$
\begin{aligned}
& \mathbf{x}_{1,1}=\mathbf{x}_{2}+\delta_{1} \theta_{2} \\
& z_{1,1}=z_{2} \\
& \theta_{1,1}=\theta_{2}
\end{aligned}
$$

or in matrix form

$$
\left\{\begin{array}{l}
x_{1,2} \\
z_{1,1} \\
\theta_{1,1}
\end{array}\right\}=\left[\begin{array}{lll}
1 & 0 & \delta_{1} \\
0 & 1 & 0 \\
0 & 0 & 1
\end{array}\right]\left\{\begin{array}{l}
x_{2} \\
z_{2} \\
\theta_{2}
\end{array}\right\}
$$

Similar relationships apply at the node pairs $(5, \mathrm{~S} 1),(8, \mathrm{P} 2)$, and $(11, \mathrm{~S} 2)$, respectively. The additional coordinates introduced at the boundary nodes are not independent but are related to the interior coordinates by relationships of the type given by Equation (4). Therefore, for free-free 


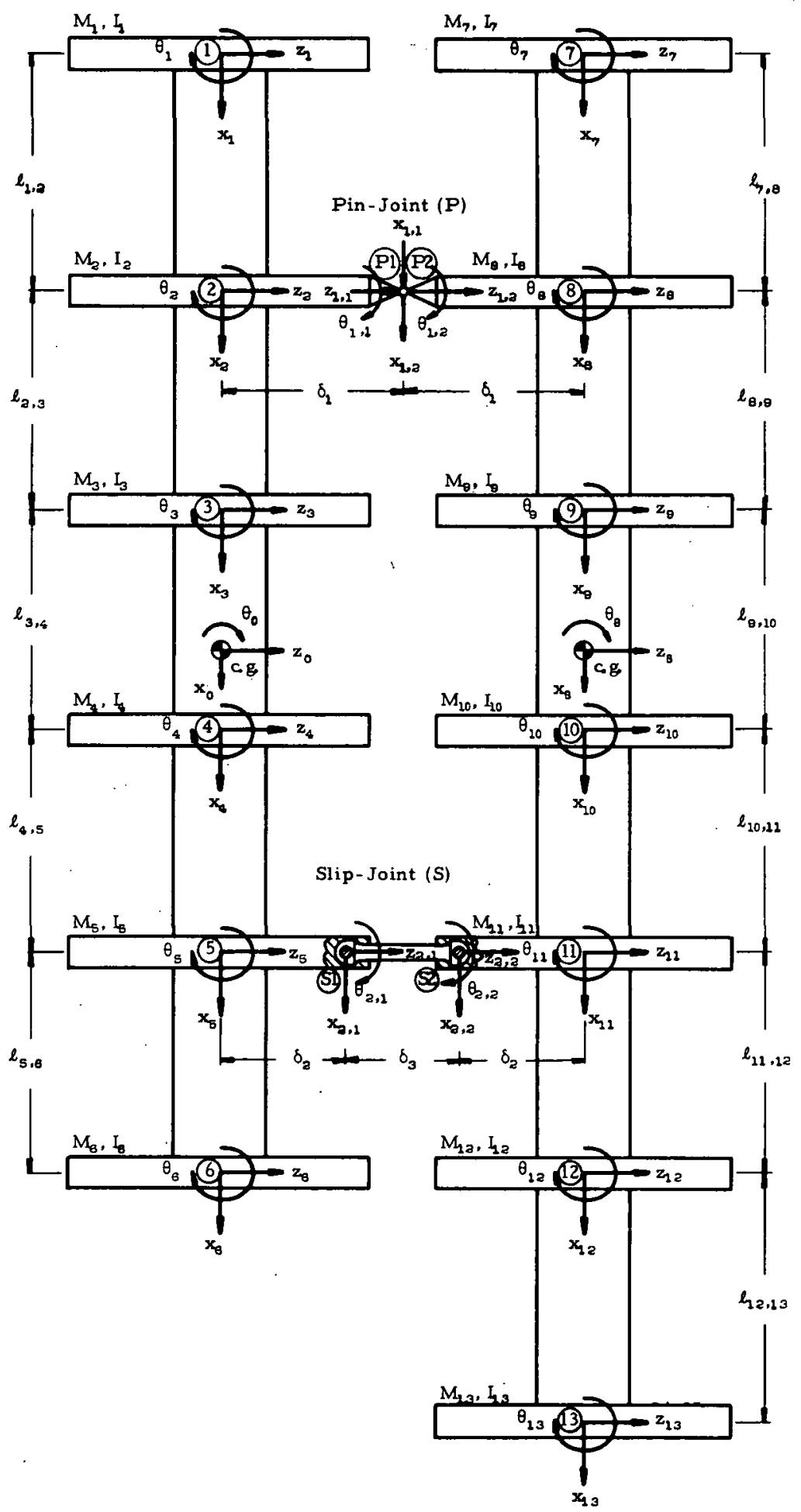

Figure 4. Coordinate System 
components, the total number of Booster degrees-of-freedom is $7 \times 3=21$ and the number of Orbiter degrees-of-freedom is $6 \times 3=18$.

In order to formulate the equations of motion for the substructures it is necessary to establish some basic relationships which will be used throughout the remainder of this report unless otherwise noted. Let each triplet of independent coordinates $\left(x_{1}, z_{1}, \theta_{1}\right)$ corresponding to the interior nodes be denoted by a matrix vector of the form

$$
\left\{\bar{u}_{1}\right\}=\left\{\begin{array}{l}
x_{1} \\
z_{1} \\
\theta_{1}
\end{array}\right\}
$$

For either the Booster or the Orbiter the complete displacement vector may then be written as

$$
\{\bar{u}\}=\left\{\begin{array}{c}
\bar{u}_{1} \\
\bar{u}_{2} \\
\vdots \\
\bar{u}_{m}
\end{array}\right\}
$$

where $m=7$ for the Booster and $m=6$ for the Orbiter.

In general, the total displacement at a node will be the sum of three displacements: ${ }^{(2)}$

(a) A displacement component corresponding to a rigid-body rotation and rigid-body translations

(b) A displacement component corresponding to constraint modes in the case where redundant constraints are present

(c) A displacement component corresponding to the natural or normal elastic modes of the structure.

Since the constraints for our Booster and Orbiter models are statically determinant for both the pin-slip and free-free cases, modes associated with redundant constraints are not applicable. Therefore, the total dis placement at a node is comprised solely of rigid-body and/or elastic normal mode displacements. In light of this, Equations (5) and (6) may be written as

$$
\left\{\bar{u}_{1}\right\}=\left\{\begin{array}{c}
x_{1} \\
z_{1} \\
\theta_{1}
\end{array}\right\}=\left\{\begin{array}{l}
x_{1} \\
z_{1} \\
\theta_{1}
\end{array}\right\}^{R}+\left\{\begin{array}{l}
x_{1} \\
z_{1} \\
\theta_{1}
\end{array}\right\}^{N}
$$


and

$$
\{\bar{u}\}=\left\{\begin{array}{c}
\overline{\mathrm{u}}_{1} \\
\overline{\mathrm{u}}_{2} \\
\vdots \\
\overline{\mathrm{u}}_{\mathrm{m}}
\end{array}\right\}=\left\{\begin{array}{c}
\overline{\mathrm{u}}_{1}{ }^{R} \\
\overline{\mathrm{u}}_{2}{ }^{2} \\
\vdots \\
\overline{\mathrm{u}}_{\mathrm{m}}{ }^{R}
\end{array}\right\}+\left\{\begin{array}{c}
\overline{\mathrm{u}}_{1}{ }^{N} \\
\overline{\mathrm{u}}_{2} \\
\vdots \\
\overline{\mathrm{u}}_{\mathrm{m}}{ }^{N}
\end{array}\right\}
$$

or

$$
\{\bar{u}\}=\left\{\bar{u}^{R}\right\}+\left\{\bar{u}^{N}\right\}
$$

where

$\mathrm{R}$ denotes rigid-body displacement

$\mathrm{N}$ denotes normal elastic mode displacement

The displacements on the right-hand side of Equation (9) may be defined in terms of a set of normalized displacement functions or modes and a set of generalized displacements. Thus

$$
\begin{aligned}
& \left\{\bar{u}^{R}\right\}=\left[\bar{\phi}^{R}\right]\left\{p^{R}\right\} \\
& \left\{\bar{u}^{N}\right\}=\left[\bar{\phi}^{N}\right]\left\{p^{N}\right\}
\end{aligned}
$$

where the element $\bar{\phi}_{1 j}$ in the modal matrices is the displacement at point $i$ in the $j$-th mode. If these equations are substituted into Equation (9), the total displacement column may be written as

$$
\{\bar{u}\}=\left[\bar{\phi}^{R}\right]\left\{p^{R}\right\}+\left[\bar{\phi}^{N}\right]\left\{p^{N}\right\}=[\bar{\phi}]\{p\}
$$

where

$$
[\bar{\phi}]=\left[\bar{\phi}^{R}: \bar{\phi}^{N}\right]
$$

and

$$
\{p\}=\left\{\begin{array}{c}
p^{R} \\
p^{N}
\end{array}\right\}
$$

From Equation (4) we note that for our model the boundary displacements may be expressed in terms of the interior displacements at a given node. Conversely, if we select the boundary displacements as the independent variables then we may write 


$$
\left\{\begin{array}{l}
x_{2} \\
z_{2} \\
\theta_{2}
\end{array}\right\}=\left[\begin{array}{lll}
1 & 0 & \delta_{1} \\
0 & 1 & 0 \\
0 & 0 & 1
\end{array}\right]^{-1}\left\{\begin{array}{l}
x_{1,1} \\
z_{1,1} \\
\theta_{1,1}
\end{array}\right\}
$$

or

$$
\left\{\bar{u}_{2}\right\}=\left[\alpha_{1,1}\right] \quad\left\{\bar{u}_{1,1}\right\}
$$

At the other boundary nodes we have similarly the relationships

$$
\begin{aligned}
& \left\{\bar{u}_{5}\right\}=\left[\alpha_{2,1}\right]\left\{\bar{u}_{2,1}\right\} \\
& \left\{\bar{u}_{8}\right\}=\left[\alpha_{1,2}\right]\left\{\bar{u}_{1,2}\right\} \\
& \left\{\bar{u}_{11}\right\}=\left[\alpha_{2,2}\right]\left\{\bar{u}_{2,2}\right\}
\end{aligned}
$$

where $\left[\alpha_{2,1}\right] \ldots\left[\alpha_{2,2}\right]$ may be determined from the kinematic constraints between the nodes. Using Equations (15) and (16a,b,c) and the node-point designations in Figure 4, we may write the following relationship for the Orbiter

$$
\left[\begin{array}{l}
\overline{\mathrm{u}}_{1} \\
\overline{\mathrm{u}}_{2} \\
\overline{\mathrm{u}}_{3} \\
\overline{\mathrm{u}}_{4} \\
\overline{\mathrm{u}}_{5} \\
\overline{\mathrm{u}}_{6}
\end{array}\right]_{0}=\left[\begin{array}{ccccc}
\mathrm{I} & & & & \\
& \alpha_{1,1} & & & 0 \\
& & \mathrm{I} & & \\
& & \mathrm{I} & & \\
& 0 & & \alpha_{2,1} & \\
& & & & I
\end{array}\right]_{0}\left[\begin{array}{l}
\overline{\mathrm{u}}_{1} \\
\overline{\mathrm{u}}_{1,1} \\
\overline{\mathrm{u}}_{3} \\
\overline{\mathrm{u}}_{4} \\
\overline{\mathrm{u}}_{2,1} \\
\overline{\mathrm{u}}_{6}
\end{array}\right]_{0}
$$

or similar to Equation (9)

$$
\{\bar{u}\}_{0}=[\alpha]_{0}\{\hat{u}\}_{0}=[\alpha]_{0}\left(\left\{\hat{u}^{R}\right\}_{0}+\left\{\hat{u}^{N}\right\}_{0}\right)
$$

Also for the Booster we have

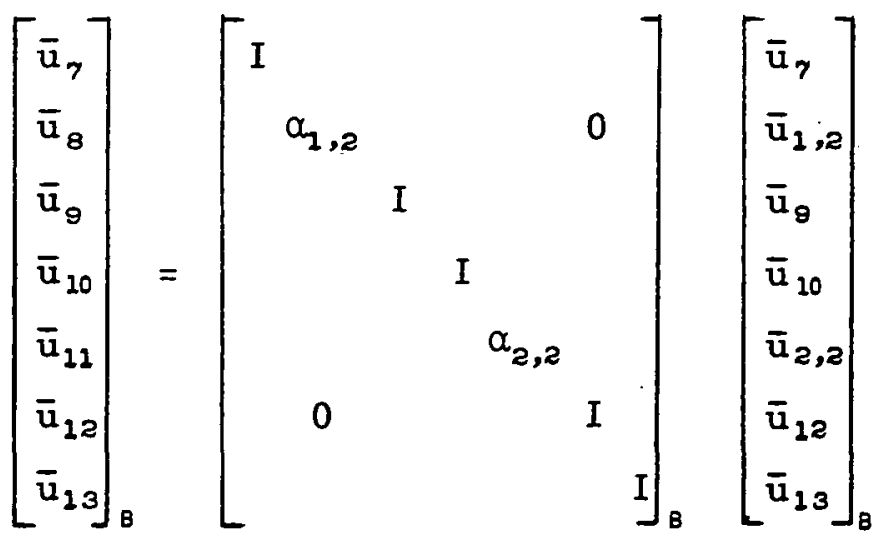


or again similar to Equation (9)

$$
\{\bar{u}\}_{B}=[\alpha]_{B}\{\hat{u}\}_{B}=[\alpha]_{B}\left(\left\{\hat{u}^{R}\right\}_{B}+\left\{\hat{u}^{N}\right\}_{B}\right)
$$

The matrices $\{\hat{u}\}_{g}$ and $\{\hat{u}\}_{0}$ now include displacements at the two boundary nodes as well as at the $n-2$ interior nodes ( $n=$ number of interior nodes for the component).

Equations (18) and (20) can be expressed as one equation with the understanding that subscript $B$ applies to the Booster only and that subscript $O$ applies to the Orbiter only. Hence,

$$
\{\bar{u}\}_{B, 0}=[\alpha]_{B, 0}\{\hat{u}\}_{B, 0}
$$

For both the Booster and Orbiter these displacements can also be expressed in terms of a set of normalized displacement functions and a set of generalized displacements. Thus

$$
\begin{aligned}
& \left\{\hat{u}^{R}\right\}=\left[\hat{\phi}^{R}\right]\left\{p^{R}\right\} \\
& \left\{\hat{u}^{N}\right\}=\left[\hat{\phi}^{N}\right]\left\{p^{N}\right\}
\end{aligned}
$$

For either the Booster or Orbiter we then have from Equation (20):

$$
\begin{aligned}
\{\bar{u}\}_{B, 0} & =[\alpha]_{B, 0}\left(\left[\hat{\phi}^{R}\right]_{B, 0}\left\{p^{R}\right\}_{B, 0}+\left[\hat{\phi}^{N}\right]_{B, 0}\left\{p^{N}\right\}_{B, 0}\right) \\
& =[\alpha]_{B, 0}[\hat{\phi}]_{B, 0}\{p\}_{B, 0}
\end{aligned}
$$

where again as in Equations (12) and (13)

$$
[\hat{\phi}]_{B, 0}=\left[\hat{\phi}^{R}: \hat{\phi}^{N}\right]_{B, 0}
$$

and

$$
\{p\}_{B, 0}=\left\{\begin{array}{c}
p^{R} \\
-p^{N}
\end{array}\right\}_{B, O}
$$

For the free-free eigenvalue problem it is simpler to use the $\{\bar{u}\}$ coordinate system and for the pin-slip eigenvalue problem the $\{\hat{u}\}$ coordinate system. A development of the component eigenvalue problems follows. 


\section{B. Component Eigenvalue Problems}

1. Pin-Slip Configurations. A finite element approach was used in deriving the stiffness matrix for each component. The component was discretized into 6 elements for the Booster and 5 elements for the Orbiter with the length of each element taken as the distance between the interior nodes as shown in Figure 4. A derivation of the element stiffness matrix for an arbitrary element with length $l_{1, g}$ is given in Appendix B. Assembly of the element stiffness matrices results in the global stiffness matrix $[\overline{\mathrm{K}}]$ for the component. The concentrated masses and moments of inertia as well as the distributed mass of the tube are lumped at the interior nodes. This results in a global mass matrix $[\overline{\mathrm{M}}]$ for the component. Since these stiffness and mass matrices are expressed solely in terms of the interior coordinate system we now include the boundary nodes by equating the internal and kinetic energies of the components in the two systems [Equations (11) and (24)]. For either component and mass configuration, equating strain energies in the two systems gives

$$
\mathrm{U}_{B, 0}=\frac{1}{2}\lfloor\hat{\mathrm{u}}\rfloor_{B, O}[\hat{\mathrm{K}}]_{B, O}\{\hat{\mathrm{u}}\}_{B, 0}=\frac{1}{2}\lfloor\overline{\mathrm{u}}\rfloor_{B, 0}[\overline{\mathrm{K}}]_{B, 0}\{\overline{\mathrm{u}}\}_{B, 0}
$$

Substituting Equation (21) into the right-hand side of Equation (27) gives

$$
\frac{1}{2}[\hat{u}]_{B, 0}[\hat{K}]_{B, 0}\{\hat{u}\}_{B, 0}=\frac{1}{2}\lfloor\hat{u}]_{B, 0}[\alpha]_{B, 0}^{\top}[\bar{K}]_{B, O}[\alpha]_{B, 0}\{\hat{u}\}_{B, 0}
$$

from which we obtain the relationship

$$
[\hat{\mathrm{K}}]_{B, 0}=[\alpha]_{B, 0}^{\top}[\overline{\mathrm{K}}]_{B, 0}[\alpha]_{B, 0}
$$

Similarly by equating the kinetic energies in the two systems we have

$$
[\hat{\mathrm{M}}]_{b, 0}=[\alpha]_{B, 0}^{\top}[\overline{\mathrm{M}}]_{b, 0}[\alpha]_{b, 0}
$$

For both the Booster and Orbiter component pin-slip configurations the boundary conditions at the joints (boundary nodes) are as follows:

$$
\begin{aligned}
& \text { Booster: } \quad \mathbf{x}_{1,2}=z_{1,2}=0 \text { at pin-joint } \\
& z_{a, z}=0 \text { at slip-joint } \\
& \text { Orbiter: } \quad x_{1,1}=z_{1,1}=0 \text { at pin-joint } \\
& z_{z, 1}=0 \text { at slip-joint }
\end{aligned}
$$

The stiffness and mass matrices are now partitioned into the following submatrices: 


$$
\begin{aligned}
& {\left[\hat{\mathrm{K}}_{b, 0} \rightarrow\left[\begin{array}{c:c}
\hat{\mathrm{K}}_{p q} & \hat{\mathrm{K}}_{q c} \\
\hdashline \hat{\mathrm{K}}_{c t} & \hat{\mathrm{K}}_{c c}
\end{array}\right]_{\mathrm{B}, 0}\right.} \\
& {\left[\hat{\mathrm{M}}_{b, 0} \rightarrow\left[\begin{array}{c:c}
\hat{\mathrm{M}}_{q p} & \hat{\mathrm{M}}_{q c} \\
\hdashline \hat{\mathrm{M}}_{c p} & \hat{\mathrm{M}}_{c c}
\end{array}\right]_{\mathrm{B}, 0}\right.}
\end{aligned}
$$

The elements in the displacement matrix $\{\hat{u}\}_{B, 0}$ are also partitioned so that the elements corresponding to the constrained boundary displacements [Equations $(31 a, b)$ and $(32 a, b)$ ] form one submatrix and the free displacements form another submatrix. Thus

$$
\{\hat{u}\}_{B, O} \rightarrow\left\{\begin{array}{c}
\hat{q}_{t} \\
-\hat{q}_{c}
\end{array}\right\}_{B, O}
$$

where

$$
\begin{aligned}
& \text { f denotes the free displacements } \\
& \text { c denotes the constrained displacements } \\
& \rightarrow \text { means replaced by. }
\end{aligned}
$$

The equations of motion for the undamped component undergoing free vibration are therefore given by

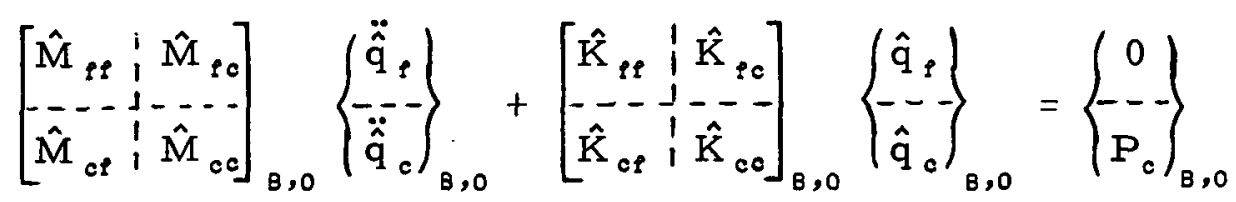

Noting now that the submatrices $\left\{\ddot{\hat{q}}_{\mathrm{c}}\right\}_{\mathrm{g}, 0}$ and $\left\{\hat{\mathrm{q}}_{\mathrm{c}}\right\}_{\mathrm{g}, 0}$ are zero due to the boundary conditions, it follows from Equation (35) that

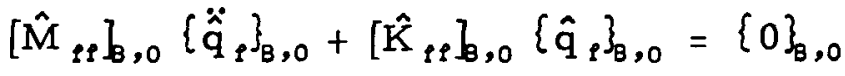

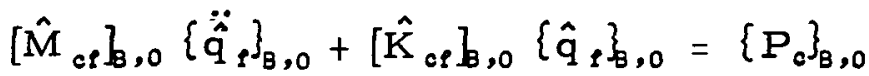

Equation (36b) gives the boundary reaction forces once the displacements at the free coordinates are known. Assuming simple harmonic motion of the form 


$$
\left\{\hat{q}_{\ell}\right\}_{B, 0}=\left\{\hat{q}_{1}^{o}\right\}_{\theta, 0} e^{i \omega t}
$$

and substituting into Equation (36a) we obtain the standard eigenvalue problem for the pin-slip boundary conditions

$$
\left|\omega^{2}[I]-[D]\right|=0
$$

where

$$
[D]=\left[\hat{M}_{\ell \uparrow}\right]_{B, 0}^{-2}\left[\hat{K}_{\ell \rho}\right]_{B, 0}
$$

A modified Jacobi method was used to obtain the eigenvalues and mode shapes corresponding to Equation (38). The mode shapes are normalized to unity displacement at the maximum displacement in each mode and are coupled in the axial and transverse directions due to the pin-slip boundary conditions. Only the transverse displacements are given in this report, a detailed description of which is given in Section V. Since all rigid-body degrees-of-freedom have been suppressed, the modes obtained are the elastic natural modes of the component.

The generalized stiffness and mass matrices may now be written as

$$
\left[\hat{K}^{N N}\right]_{B, O}=\left[\hat{\phi}_{P:}^{N}: \hat{\phi}_{c}^{N}=0\right]_{B, O}\left[\begin{array}{l:l}
\hat{K}_{P} & \hat{K}_{P c} \\
\hdashline \hat{K}_{c} & \hat{K}_{c c}
\end{array}\right]_{B, O}\left[\begin{array}{l}
\hat{\phi}_{t}^{N} \\
\hat{\phi}_{c}^{N}=0
\end{array}\right]_{B, O}
$$

where

$$
\begin{array}{ll}
{\left[\hat{\phi}_{f}^{N}\right] \quad \begin{array}{l}
\text { is a matrix of normalized modal displacements for } \\
\text { the unconstrained coordinates }
\end{array}} \\
{\left[\hat{\phi}_{c}^{\mathrm{N}}\right]=0 \quad \begin{array}{l}
\text { is a null matrix since the displacements are zero } \\
\text { at the constrained coordinates. }
\end{array}}
\end{array}
$$

and

$$
\left.\left[\hat{m}^{N N}\right]_{B, 0}=\left[\hat{\phi}_{t}^{N}: \hat{\phi}_{c}^{N}=0\right]\right]_{B, 0}\left[\begin{array}{l:l}
\hat{M}_{p:} & \hat{M}_{\rho c} \\
\hdashline \hat{M}_{c t} & \hat{M}_{c c}
\end{array}\right]\left[\begin{array}{l}
\hat{\phi}_{\ell}^{N} \\
\hdashline \hat{\phi}_{c}^{N}=0
\end{array}\right]
$$

2. Free-Free Configurations. The component free-free configuration eigenvalue problem can be set up in two different ways which we denote as the direct method and the transformation method. Both methods are required for subsequent combined System analysis, and will be included here to provide an indication of numerical accuracy in 
component analysis. The transformation method requires a knowledge of the pin-slip modes in order to determine the free-free modes. Since in the pin-slip configurations the rigid-body modes have been suppressed, we must now synthesize these modes into the equations of motion. This is necessary for both the component case and for the System case where pin-slip modes are used as generalized displacements.

a. Direct Method. In the direct method the stiffness and

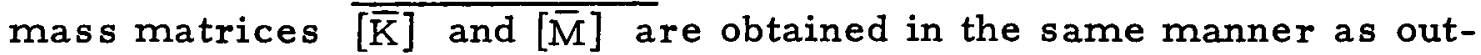
lined in Section B. 1 for the pin-slip configurations. These matrices are written in the $\{\bar{u}\}$ coordinate system with the displacements defined at the component interior nodes. Since there a re no boundary constraints for the free-free configurations the component undamped equations of free motion are

$$
[\overline{\mathrm{M}}]_{\mathrm{B}, \mathrm{O}}\{\overline{\overline{\mathrm{u}}}\}_{\mathrm{B}, \mathrm{O}}+[\overline{\mathrm{K}}]_{\mathrm{B}, \mathrm{O}}\{\overline{\mathrm{u}}\}_{\mathrm{B}, 0}=\{0\}
$$

Assuming simple harmonic motion of the form

$$
\{\bar{u}\}_{B, 0}=\{\bar{u} 0\}_{B, 0} e^{i \omega t}
$$

and substituting into Equation (42) we obtain the standard eigenvalue problem for the free-free boundary conditions

$$
\left|\omega^{2}[I]-[D]\right|=0
$$

where

$$
[D]=[\overline{\mathrm{M}}]_{B, 0}^{-1}[\overline{\mathrm{K}}]_{B, 0}
$$

A modified Jacobi method was used to obtain the eigenvalues and mode shapes corresponding to Equation (45). The mode shapes are normalized to unity displacement at the maximum displacement in each mode and are uncoupled in the axial and transverse directions. Since we have considered only planar motion in this study, we obtain three rigid-body modes in addition to the elastic natural modes of the component. A detailed description of the free-free component natural modes and frequencies is given in Section V.

The generalized stiffness and mass matrices for free-free components may now be written as

$$
[\bar{X}]_{B, 0}=\left[\begin{array}{c:c}
0 & 0 \\
\hdashline 0 & \bar{X}_{B, O}^{N N}
\end{array}\right]_{0}=\left[\begin{array}{c}
\bar{\phi}^{R} \\
\hdashline \bar{\phi}^{N}
\end{array}\right]_{B, 0}[\bar{K}]_{B, 0}\left[\bar{\phi}^{R}: \bar{\phi}^{N}\right]_{B, O}
$$

and 


$$
[\bar{m}]_{B, 0}=\left[\begin{array}{c:c}
\bar{m}^{R R} & 0 \\
\hdashline 0 & \bar{m}_{B, 0}^{R R}
\end{array}\right]_{B, 0}=\left[\begin{array}{c}
\bar{\phi}^{R} \\
\hdashline \bar{\phi}^{N}
\end{array}\right]_{B, 0}[\bar{M}]_{B, 0}\left[\bar{\phi}^{R}: \bar{\phi}^{N}\right]
$$

where

$\left[\bar{\phi}^{R}\right]$ is the matrix of normalized rigid-body displacements

$\left[\bar{\phi}^{N}\right]$ is the matrix of normalized elastic modal displacements

and both are synonomous with the modal functions defined in Equations $(10 a, b)$. It should be noted that all submatrices in the generalized stiffness matrix associated with rigid-body displacements are null since no internal work is done during a rigid-body displacement.

b. Transformation Method. In the transformation method we attempt to obtain the free-free component eigenvalues and mode shapes by using our analytically derived pin-slip modes and by appropriately including rigid-body motion in the equations of motion. Recall that in Section III. B. 1 we obtained the pin-slip modal matrix

$$
\left[\hat{\phi}^{N}\right]=\left[\begin{array}{c}
\hat{\phi}_{p}^{N} \\
\hdashline \hat{\phi}_{c}^{N}
\end{array}\right]
$$

where mode $j$ is specified in column $j$ of this modal matrix and includes displacements at the boundary nodes as well as at the interior nodes. We now have to include rigid-body motion with the pin-slip modes. Let us therefore define in the $\{\bar{u}\}$ system three rigid-body modes in the axial-a, lateral- $\ell$, and rotational-r, directions, respectively, such that the rigidbody displacements are related to the modes and generalized displacements by the following relationships:

$$
\left\{\bar{u}_{a}\right\}=\left\{\bar{\phi}_{a}\right\}_{p_{a}}=\left\{\begin{array}{c}
1 \\
0 \\
0 \\
-\frac{1}{1} \\
0 \\
0 \\
\hdashline \vdots \\
\vdots \\
--- \\
1 \\
0 \\
0
\end{array}\right\} p_{a}
$$




$$
\begin{aligned}
& \left\{\bar{u}_{\ell}\right\}=\left\{\bar{\phi}_{l}\right\} \mathrm{p}_{\ell}=\left(\begin{array}{c}
0 \\
1 \\
0 \\
\hdashline 0 \\
1 \\
0 \\
-\vdots \\
\vdots \\
-\vdots \\
0 \\
1 \\
0
\end{array}\right)--p_{l} \\
& \left\{\bar{u}_{r}\right\}=\left\{\bar{\phi}_{r}\right\} p_{r}=\left\{\begin{array}{c}
0 \\
z_{2}^{*} \\
1 / \ell \\
\hdashline 0 \\
z_{2}^{*} \\
1 / \ell \\
-\vdots \\
\vdots \\
-\vdots \\
0 \\
z_{z}^{*} \\
1 / \ell
\end{array}\right\} p_{r}
\end{aligned}
$$

where $\mathrm{p}_{\mathrm{a}}$, $\mathrm{p}_{\ell}$, and $\mathrm{p}_{\mathrm{r}}$ are the respective generalized coordinates. In Equation (49c) a normalized rotational mode is selected such that a rotation of $\theta=1 / \ell$ about the component center-of-gravity produces a unit displacement at the tip of the component and $l$ is the distance between the $\mathrm{cg}$ and the tip node. Thus, $z_{j}^{*}$ is the displacement in the lateral direction at interior node $j$ due to a rotation about the component $c g$ equal to $\theta=1 / l$. For both the Booster and Orbiter, $z_{m}^{*}$ was equal to 1 at the bottom node. Equations $(49 a, b, c)$ may be combined so that

$$
\left\{\bar{u}^{R}\right\}=\left[\begin{array}{lll}
\bar{\phi}_{a} & \bar{\phi}_{l} & \bar{\phi}_{r}
\end{array}\right]\left\{\begin{array}{c}
p_{a} \\
\hdashline p_{l} \\
\hdashline p_{r}
\end{array}\right\}=\left[\bar{\phi}^{R}\right]\left\{p^{R}\right\}
$$

From Equation (21) we had that

$$
\{\bar{u}\}_{B, 0}=[\alpha]_{B, 0}\{\hat{u}\}_{B, O}
$$

If we multiply both sides of Equation $(50)$ by $[\alpha]_{B, 0}^{-1}$ we have

$$
[\alpha]_{B, 0}^{-1}\left\{\bar{u}^{R}\right\}=[\alpha]_{B, 0}^{-1}\left[\bar{\phi}^{R}\right]\left\{p^{R}\right\}
$$


or

$$
\left\{\hat{u}^{R}\right\}=\left[\hat{\phi}^{R}\right]\left\{p^{R}\right\}
$$

which conforms with Equation (22). Equation (52) now gives the rigid-body displacements in the $\{\hat{u}\}$ coordinate system. Similar to the normal mode matrix $\left[\hat{\phi}^{N}\right]$ the elements in $\left[\hat{\phi}^{R}\right]$ are arranged as indicated by Equation (48) so that

$$
\left[\hat{\phi}^{R}\right] \rightarrow\left[\begin{array}{c}
\hat{\phi}_{t}^{R} \\
-\hat{\phi}_{c}^{R}
\end{array}\right]
$$

where again

$\rightarrow \quad$ means replaced by.

Combining Equations (48) and (53) into one equation we have for both the Booster and the Orbiter

$$
[\hat{\phi}]_{B, 0}=\left[\hat{\phi}^{R}: \hat{\phi}^{N}\right]_{B, 0}
$$

and

$$
[\hat{\mathrm{u}}]_{B, 0}=[\hat{\phi}]_{B, 0}\{\mathrm{p}\}_{B, 0}
$$

Equation (54b) now gives the displacement at a point on the component as a linear combination of pin-slip modes and rigid-body displacements. The kinetic energy in the free-free configuration may be written as

$$
\begin{aligned}
\mathrm{T}_{B, O} & =\frac{1}{2}\lfloor\dot{\hat{u}}\rfloor_{B, O}[\hat{\mathrm{M}}]_{B, O}\{\dot{\hat{\mathrm{u}}}\}_{B, O} \\
& =\frac{1}{2}\lfloor\dot{\mathrm{p}}\rfloor_{B, O}[\hat{\phi}]_{B, O}^{\top}[\hat{\mathrm{M}}]_{B, O}[\hat{\phi}]_{B, O}\{\dot{\mathrm{p}}\}_{B, O}
\end{aligned}
$$

from which we define the mass matrix

$$
[\hat{\mathrm{m}}]_{B, 0}=\left[\begin{array}{c:c}
\hat{\mathrm{m}}^{\mathrm{RR}} & \hat{\mathrm{m}}^{\mathrm{RN}} \\
\hdashline \hat{\mathrm{m}}^{\mathrm{NR}} & \hat{m}^{\mathrm{NN}}
\end{array}\right]_{\mathrm{B}, 0}
$$

Note here that the matrix $\left[\hat{m}^{N N}\right]$ is already available from the pin-slip analysis and is given by Equation (41). The matrix $\left[\hat{\mathrm{m}}^{\mathrm{RR}}\right]$ represents the rigid-body masses while the matrices $\left[\hat{\mathrm{m}}^{\mathrm{RN}}\right]$ and $\left[\hat{\mathrm{m}}^{\mathrm{NR}}\right]$ represent the coupling masses. Using the stiffness matrix derived in Equation (40) we may now write the equations of motion for the undamped free-free components as 


$$
\left[\begin{array}{c:c}
\hat{m}^{R R} & \hat{m}^{R N} \\
\hdashline \hat{m}^{N R} & \hat{m}^{N N}
\end{array}\right]_{B, 0}\left\{\begin{array}{c}
\ddot{p}^{R} \\
\hdashline \ddot{p}^{N}
\end{array}\right\}_{B, 0}+\left[\begin{array}{c:c}
0 & 0 \\
\hdashline 0 & \hat{x}^{N N}
\end{array}\right]_{B, O}\left\{\begin{array}{c}
p^{R} \\
\hdashline p^{N}
\end{array}\right\}_{B, 0}=\{0\}
$$

or

$$
[\hat{\mathrm{m}}]_{B, 0}\{\ddot{\mathrm{p}}\}_{B, O}+[\hat{\mathrm{k}}]_{B, 0}\{\mathrm{p}\}_{B, 0}=\{0\}
$$

Assuming simple harmonic motion the eigenvalues and mode shapes corresponding to Equation (57) were determined by a modified Jacobi method for the Booster component using the first six (6) pin-slip modes and three (3) rigid-body modes as generalized displacements. Eigenvalue results of the analysis are presented in Table II and compared with the results obtained by the direct method.

TABLE II. COMPARISON OF NATURAL FREQUENCIES OBTAINED BY DIRECT AND TRANSFORMATION METHODS

\begin{tabular}{|c|c|c|c|}
\hline $\begin{array}{c}\text { Mode } \\
\text { No. }\end{array}$ & $\begin{array}{c}\text { Natural Frequency } \\
\text { Direct Method }\end{array}$ & $\begin{array}{c}\text { Natural Frequency } \\
\text { Transformation Method }\end{array}$ & $\% \Delta^{*}$ \\
\hline 1 & 8.53 & 8.54 & +0.12 \\
2 & 24.08 & 24.13 & +0.21 \\
3 & 45.72 & 45.60 & -0.26 \\
4 & 75.25 & 75.32 & +0.09 \\
5 & 106.12 & 106.29 & +0.16 \\
6 & 203.39 & --- & -- \\
\hline
\end{tabular}

Eigenvector results can also be used to define the following generalized stiffness and mass matrices

$$
\left.[\hat{X}]_{B, 0}=\left[\begin{array}{c}
\varphi^{R} \\
\hdashline \varphi_{B, 0}^{N}
\end{array}\right]_{B, 0}[\hat{k} \cdot] \hat{\varphi}_{B, 0}^{R} \hat{\varphi}^{N}\right]
$$

and

$$
\left[\hat{m}_{B, O}=\left[\begin{array}{c}
\hat{\varphi}^{R} \\
\hdashline \hat{\varphi}_{B, O}^{N}
\end{array}\right]_{B, 0}[\hat{m}]\left[\hat{\varphi}^{R}: \hat{\varphi}^{N}\right]\right.
$$


where

$\left[\hat{\varphi}^{R}\right]$ is the matrix of normalized rigid-body displacements
$\left[\hat{\varphi}^{N}\right]$ is the matrix of normalized elastic modal displacements. Now since the same component is represented in both the direct and transformation methods of analysis we must have

$$
\begin{aligned}
& {[\bar{x}]_{B, 0}=[\hat{K}]_{B, O}} \\
& {[\bar{m}]_{B, O}=[\hat{m}]_{B, 0}}
\end{aligned}
$$

Use of these equations allows a check on the numerical accuracy in the problem, and is particularly useful for checking whether a sufficient number of elastic modes have been used in the analysis.

As can be seen from Table II, the natural frequencies obtained by the transformation method are in excellent agreement with those obtained by the direct method through the fifth mode. Inspection of the mode shapes also showed excellent agreement although they are not presented here. Inclusion of rigid-body modes in the pin-slip component mode synthesis for the combined System follows the same procedure as outlined in the transformation method. The results obtained from this example provided a good qualitative and quantitative indication of results to be expected in the System analysis which is discussed in Section III. D.

\section{Component Forced Response}

The component forced response analysis was based on the wellknown assumption of modal damping properties which is valid for systems having light damping and well separated modes. That is, at resonance, the response is due principally to a single mode. More will be said on this matter in a later section. For the present discussion, however, we take the damping to be viscous and proportional to the linear critical damping for each component mode. From damping measurements made in the laboratory on each component we obtain a diagonal matrix of damping coefficients which represent the percentage of critical damping for each elastic mode. In addition to the modal damping we also measure the damping obtained during rigid-body motion at very low frequencies, since in the free-free configurations this damping will be present due to the damping mechanism employed in the experiments. Note that for a real vehicle this rigid-body damping might correspond to low frequency aerodynamic damping.

Assuming linear superposition of the modal displacements in the 


$$
\begin{aligned}
& \{\hat{u}\}_{B, O}=\left[\hat{\phi}^{N}\right]_{B, O}\left\{p^{N}\right\}_{B, O} \quad \text { pin-slip case } \\
& \{\bar{u}\}_{B, O}=\left[\begin{array}{l:l}
\bar{\phi}^{R} & \left.\bar{\phi}^{N}\right]_{B, O}\left\{\begin{array}{c}
p^{R} \\
\hdashline p^{N}
\end{array}\right\}_{B, O} \quad \text { free-free case }
\end{array}\right.
\end{aligned}
$$

we may, by orthogonality of the normal modes, write the equations of motion for forced response as

$$
\begin{aligned}
& {\left[\begin{array}{c:c}
m^{R R} & 0 \\
\hdashline 0 & m^{N N}
\end{array}\right]_{B, O}\left\{\begin{array}{l}
\ddot{p}^{R} \\
\hdashline \ddot{p}^{N}
\end{array}\right\}_{B, O}+\left[\begin{array}{c:c}
C^{R R} & 0 \\
\hdashline 0 & c^{N N}
\end{array}\right]_{B, O}\left\{\begin{array}{l}
\dot{p}^{R} \\
\hdashline \dot{p}^{N}
\end{array}\right\}_{B, O}} \\
& +\left[\begin{array}{c:c}
0 & 0 \\
\hdashline 0 & \omega^{N N} m^{N N}
\end{array}\right]_{B, O}\left\{\begin{array}{c}
p^{R} \\
\hdashline p^{N}
\end{array}\right\}_{B, O}=\left\{\begin{array}{c}
\mathscr{F}^{R} \\
\hdashline \mathscr{F}^{N}
\end{array}\right\}_{B, O} \sin \bar{\omega}_{t}
\end{aligned}
$$

which holds for both the pin-slip and free-free cases so long as the appropriate matrix elements are utilized. Further, all the square matrices on the left side of Equation (62) are diagonal and the damping matrix is given by.

$$
\left[\begin{array}{c:c}
C^{R R} & 0 \\
\hdashline 0 & C^{N N}
\end{array}\right]_{B, 0}=2\left[\begin{array}{c:c}
m^{R R} & 0 \\
\hdashline 0 & m^{N N}
\end{array}\right]_{B, O}\left[\begin{array}{c:c}
\omega^{R R} & 0 \\
\hdashline 0 & \omega^{N N}
\end{array}\right]_{B, O}\left[\begin{array}{c:c}
5^{R R} & 0 \\
\hdashline 0 & S^{N N}
\end{array}\right]_{B, 0}
$$

where

$$
\begin{aligned}
& {\left[\begin{array}{c:c}
\omega^{R R} & 0 \\
\hdashline 0 & \omega^{N N}
\end{array}\right]_{B, 0} \begin{array}{l}
\text { square diagonal matrix of rigid-body and } \\
\text { elastic modal natural frequencies (note that } \\
{\left[\omega^{R R}\right] \text { is null). }}
\end{array}} \\
& {\left[\begin{array}{c:c}
\zeta^{R R} & 0 \\
\hdashline 0 & S^{N N}
\end{array}\right]_{B, 0}=\begin{array}{l}
\text { square diagonal matrix of rigid-body and } \\
\text { elastic modal critical damping ratios }
\end{array}} \\
& \left\{\begin{array}{l}
F^{R} \\
--F^{N}
\end{array}\right\}_{B, 0} \quad=\begin{array}{l}
\text { vector matrix of applied generalized } \\
\text { forces }
\end{array}
\end{aligned}
$$

Equation (62) represents a set of $m+3$ ( $m=$ number of elastic modes) uncoupled equations in the generalized $p$ coordinates which is applicable to both the free-free and pin-slip component configurations provided that the submatrices $m^{R R}, C^{R R}$, and $\mathscr{F}^{R}$ are set equal to zero for the pin-slip 
configurations. The $j$-th uncoupled equation of motion in the generalized coordinate corresponding to the $j$-th elastic mode may be written as

$$
\ddot{p}_{j}{ }^{N}+2 \zeta_{\jmath}{ }^{N N} w_{\jmath}{ }^{N N} \dot{p}_{j}{ }^{N}+w_{j}{ }^{N}{ }^{2} p_{j}{ }^{N}=F_{j}{ }^{N} \sin \bar{w}_{t} / m_{\jmath}{ }^{N N}
$$

For steady-state excitation a particular solution to Equation (65) is given by

$$
p_{\jmath}^{N}=A_{j}^{N} \sin \left(\bar{w} t-\Psi_{j}^{N}\right)
$$

where $\bar{\omega}$ is the forcing frequency. If we define $\Omega_{j}=\bar{w} / \omega_{j}{ }^{N N}$ and substitute Equation (66) into Equation (65) we obtain for $A_{j}^{N}$ and $\Psi_{j}^{N}$

$$
\begin{aligned}
& A_{j}^{N}=\frac{\mathscr{F}_{j}^{N} /\left(m_{j}{ }^{N N} \omega^{N N^{2}}\right)}{\left[\left(1-\Omega_{\jmath}^{2}\right)^{2}+\left(2 \zeta_{j}{ }^{N N} \Omega_{\jmath}\right)^{2}\right]^{\frac{T}{2}}} \\
& \Psi_{j}^{N}=\arctan \left(\frac{2 \zeta_{j}{ }^{N N} \Omega_{\jmath}}{1-\Omega_{j}^{2}}\right)
\end{aligned}
$$

The $j$-th uncoupled equation of motion in the generalized coordinate corresponding to the $j$-th rigid-body mode may be written as

$$
\ddot{p}_{j}{ }^{R}+2 S_{j}{ }^{R R} \bar{w} \dot{p}_{j}{ }^{R}=\mathscr{F}_{j}{ }^{R} \sin \bar{w} t / m_{j}{ }^{R R}
$$

For steady-state excitation a particular solution to Equation (69) is given by

$$
P_{j}{ }^{R}=A_{j}{ }^{R} \sin \left(\bar{\omega} t-\Psi_{j}{ }^{R}\right)
$$

Substituting Equation (70) into Equation (69) we obtain for $\mathrm{A}_{j}{ }^{R}$ and $\Psi_{j}{ }^{R}$

$$
\begin{aligned}
& A_{j}{ }^{R}=\frac{\mathscr{F}_{j}{ }^{N} /\left(m_{j}{ }^{R R} \bar{w}^{2}\right)}{\left[I+4 \zeta_{j}{ }^{2}{ }^{2}\right]^{\frac{1}{2}}} \\
& \Psi_{j}{ }^{R}=\arctan \left(-2 \zeta_{j}{ }^{R R}\right)
\end{aligned}
$$

Once the generalized coordinates have been obtained substitution into either Equation (60) or Equation (61) gives the desired displacements at the node points. Results of the component forced response analysis are presented in Section $V$ for each configuration. 


\section{System Equations}

The procedure used to develop the System modes from the Booster and Orbiter component modes es sentially follows that of Hurty (2). The component modes, as considered by Hurty, fall into three categories: rigid-body modes in which the component is displaced without deformation, modes which exist only if the system of constraints on the component is indeterminate (called "constraint modes"), and normal modes that define displacements relative to the constraint system.

In the first category, three rigid-body modes are considered for each component which consist of two rigid-body translations and a small rotation. Since the system of constraints on our model is determinate, the modes in the second category do not exist. In the third category we have normal modes that define displacements relative to a constrained system (pin-slip modes) and normal modes that are obtained with no constraints present (free-free modes).

1. Component Matrices. Since the procedure used to develop the System analysis is identical for both the pin-slip mode synthesis and the free-free mode synthesis, we will not repeat the procedure separately for each case in this report. We would, however, like to make the following distinction between the two cases which should be observed in the development of the System equations: (1) For System synthesis utilizing pin-slip modes the component mass matrix is given by

$$
[\hat{\mathrm{m}}]_{\mathrm{B}, O}=\left[\begin{array}{c:c}
\hat{\mathrm{m}}^{\mathrm{RR}} & \hat{\mathrm{m}}^{\mathrm{RN}} \\
\hdashline \hat{\mathrm{m}}^{\mathrm{NR}} & \hat{\mathrm{m}}^{\mathrm{NN}}
\end{array}\right]_{\mathrm{B}, \mathrm{O}}
$$

where the submatrices $\hat{\mathrm{m}}^{\mathrm{RR}}, \hat{\mathrm{m}}^{\mathrm{RN}}$, and $\hat{\mathrm{m}}^{\mathrm{NR}}$ are given by

$$
\begin{aligned}
& {\left[\hat{\mathrm{m}}^{\mathrm{RR}}\right]_{B, O}=\left[\hat{\phi}^{R}\right]_{B, O}^{T}[\hat{\mathrm{M}}]_{B, O}\left[\hat{\phi}^{R}\right]_{B, O}} \\
& {\left[\hat{\mathrm{m}}^{\mathrm{RN}}\right]_{B, O}=\left[\hat{\phi}^{R}\right]_{B, O}^{T}[\hat{\mathrm{M}}]_{B, 0}\left[\hat{\phi}^{N}\right]_{B, O}}
\end{aligned}
$$

and

$$
\left[\hat{\mathrm{m}}^{\mathrm{NR}}\right]_{\mathrm{B}, 0}=\left[\hat{\phi}^{N}\right]_{B, 0}^{T}[\hat{\mathrm{M}}]_{\mathrm{B}, 0}\left[\hat{\phi}^{\mathrm{R}}\right]_{\mathrm{B}, 0}
$$

The se submatrices were obtained by using the transformation procedure outlined in Section III. B.2.b. Note again that the submatrix $\left[m^{\mathrm{NN}}\right]$ is already available from the pin-slip modal analysis. (2) For System synthesis utilizing free-free modes the component generalized mass matrix is given by 


$$
[\bar{m}]_{B, 0}=\left[\begin{array}{c:c}
\bar{m}^{R R} & 0 \\
\hdashline 0 & \bar{m}^{N N}
\end{array}\right]_{B, O}
$$

where both the submatrices $\left[\bar{m}^{R R}\right]$ and $\left[\bar{m}^{N N}\right]$ are available from the free-free modal analysis [Equation (47)]. With this distinction in mind, we proceed to develop the System equations.

For the Booster we may write the equations of motion with equivalent viscous damping as

$$
[m]_{B}\{\ddot{p}\}_{B}+[C]_{B}\{\dot{p}\}_{B}+[K]_{B}\{p\}_{B}=\{F(t)\}_{B}
$$

and likewise for the Orbiter

$$
[M]_{0}\{\ddot{p}\}_{0}+[C]_{0}\{\dot{p}\}_{0}+[K]_{0}\{p\}_{0}=\{\mathscr{F}(t)\}_{0}
$$

where for pin-slip mode synthesis the mass matrix is given by Equation (73) and the stiffness matrix is given by

$$
[K]=\left[\begin{array}{c:c}
0 & 0 \\
\hdashline 0 & \hat{x}^{N N}
\end{array}\right]
$$

and the $\left[\hat{X}^{N N}\right]$ submatrix is defined in Equation (40). For the free-free mode synthesis the mass matrix is given by Equation (47) or (75) and the stiffness matrix by Equation (46). The damping matrix is a diagonal matrix of the form given by Equation (63). Equations (76) and (77) may be combined in the following uncoupled form:

$$
[m]\{\mathrm{p}\}+[C]\{\mathrm{p}\}+[K]\{\mathrm{p}\}=\{\mathscr{F}(\mathrm{t})\}
$$

where

$$
\begin{aligned}
& {[m]=\left[\begin{array}{ll}
{[m]_{B}} & {[m]_{0}}
\end{array}\right]} \\
& {[c]=\left[\begin{array}{ll}
{[c]_{B}} & {[c]_{0}}
\end{array}\right]} \\
& {[K]=\left[\begin{array}{ll}
{[K]_{B}} & {[K]_{0}}
\end{array}\right]} \\
& \{p\}=\left\{\begin{array}{l}
\{p\}_{B} \\
\{p\}_{0}
\end{array}\right\}
\end{aligned}
$$


and

$$
\{\mathscr{F}(t)\}=\left\{\begin{array}{l}
\{\mathscr{F}(t)\}_{B} \\
\{\mathscr{F}(t)\}_{0}
\end{array}\right\}
$$

Equation (79) now represents two matrix equations of motion for the unconnected Booster and Orbiter. The process of connecting the components by requiring that the displacements at their points of mutual attachment be equal is now discussed.

2. Coupling Matrices. At the pin-joint attachment point we have the following displacement compatibility requirements

$$
x_{1,2}-x_{1,2}=0 \text { and } z_{1,2}-z_{1,2}=0
$$

while at the slip-joint we have the displacement compatibility requirement that

$$
z_{2,1}-z_{2,2}=0
$$

Using the pin-slip component modes and the component rigid-body modes, the $x_{1,1}$ displacement may be written as follows:

$$
x_{1,1}=\phi_{1,1 \mathrm{a}}^{x} p_{a}^{0}+\phi_{1,2 \mathrm{r}}^{x} p_{r}^{0}+\sum_{j=1}^{m} \phi_{1,1, j}^{x} p_{j}^{0}
$$

where

$$
\begin{aligned}
& \phi_{1,1 \mathrm{a}}^{\mathrm{x}}=\begin{array}{l}
\text { normalized displacement of } \mathrm{x}_{1,1} \text { in rigid-body } \\
\text { axial mode }
\end{array} \\
& \phi_{1,1 \mathrm{r}}^{\mathrm{x}}=\text { normalized displacement of } \mathrm{x}_{1,1} \text { in rigid-body } \\
& \text { rotational mode } \\
& \phi_{1,1,}^{x}=\text { normalized displacement of } x_{1,2} \text { in the } j \text {-th } \\
& \text { elastic mode } \\
& \mathrm{p}_{\mathrm{a}}^{0}, \mathrm{p}_{\mathrm{r}}^{0}, \mathrm{p}_{\mathrm{j}}^{0}=\text { generalized coordinates on Orbiter corresponding } \\
& \text { to the axial, rotational, and } j \text {-th elastic modes, } \\
& \text { respectively. }
\end{aligned}
$$

Similarly, the remaining displacements may be written as 


$$
\begin{aligned}
& \mathrm{z}_{1,1}=\phi_{1,1 \ell}^{2} \mathrm{p}_{l}^{0}+\phi_{1,1 \mathrm{r}}^{2} \mathrm{p}_{s}^{0}+\sum_{j=1}^{\mathrm{m}} \phi_{2,1, j}^{2} \mathrm{p}_{\mathrm{j}}^{0} \\
& x_{1,2}=\phi_{1,2 a}^{x} p_{a}^{B}+\phi_{1,2_{r}}^{x} p_{r}^{B}+\sum_{j=1}^{n} \phi_{1,2, j}^{x} p_{j}^{B} \\
& \mathrm{z}_{1,2}=\phi_{1,2}^{2} \mathrm{p}_{l}^{\mathrm{B}}+\phi_{1,2 \mathrm{r}}^{2} \mathrm{p}_{\mathrm{r}}^{\mathrm{B}}+\sum_{j=1}^{\mathrm{n}} \phi_{1,2 \mathrm{j}}^{2} \mathrm{p}_{\mathrm{j}}^{\mathrm{B}} \\
& z_{2,1}=\phi_{2,1}^{2} p_{l}^{0}+\phi_{2,1, \tau}^{2} p_{I}^{0}+\sum_{j=1}^{m} \phi_{2,1, j}^{2} p_{j}^{0} \\
& z_{2,2}=\phi_{2,2 l}^{z} p_{l}^{8}+\phi_{2,2 r}^{2} p_{r}^{B}+\sum_{j=1}^{n} \phi_{2,2,}^{2} p_{j}^{8}
\end{aligned}
$$

where

$$
\begin{aligned}
& \phi_{1,1 l}^{2}=\text { normalized displacement of } z_{2,1} \text { in rigid-body } \\
& \text { lateral mode } \\
& \mathrm{p}_{\ell}^{\mathrm{B}}, \mathrm{p}_{\ell}^{0}=\begin{array}{l}
\text { generalized coordinates on Booster and Orbiter } \\
\text { corresponding to the rigid-body lateral modes }
\end{array} \\
& p_{j}^{\theta}, p_{j}^{0}=\text { generalized coordinate on Booster corresponding } \\
& \text { to the } \mathrm{j} \text {-th elastic mode }
\end{aligned}
$$

Note, however, that for the pin-slip case the elastic modal displacements at the boundary nodes were zero in the appropriate directions [Equations $(31 \mathrm{a}, \mathrm{b})$ and $(32 \mathrm{a}, \mathrm{b})]$. Therefore, all the modal displacements in the summation in Equations ( $82 \mathrm{a}$ ) through (82f) are zero since they correspond to these constrained displacements. From the compatibility requirements given by Equations $(81 \mathrm{a}, \mathrm{b})$ we obtain three equations of displacement compatibility in $m+n+6$ generalized $p$ coordinates where $m=$ number of Orbiter elastic modes and $n=$ number of Booster elastic modes used in the analysis. These equations may be written in matrix form as 


$$
\left[\begin{array}{c:c:c:c}
\hat{\phi}_{B}^{R} & 0 & \hat{\phi}_{0}^{R} & 0
\end{array}\right] \quad\left\{\begin{array}{c}
p_{B}^{R} \\
\hdashline p_{B}^{N} \\
\hdashline p_{0}^{R} \\
\hdashline p_{0}^{N}
\end{array}\right\}=0
$$

where

$$
\begin{aligned}
& {\left[\hat{\phi}_{B}^{\mathrm{R}}\right]=\left[\begin{array}{ccc}
\phi_{1,2 \mathrm{a}}^{\mathrm{x}} & 0 & -\phi_{1,2 \mathrm{x}}^{\mathrm{x}} \\
0 & \phi_{1,2 l}^{\mathrm{z}} & -\phi_{1,2 \mathrm{z}}^{\mathrm{z}} \\
0 & \phi_{2,2 l}^{\mathrm{z}} & -\phi_{2,2 \mathrm{z}}^{\mathrm{z}}
\end{array}\right]} \\
& {\left[\hat{\phi}_{0}^{R}\right]=\left[\begin{array}{ccc}
\phi_{1,12}^{x} & 0 & \phi_{1,2 r}^{x} \\
0 & \phi_{1,2 l}^{z} & \phi_{1,2 x}^{z} \\
0 & \phi_{2,1 l}^{z} & \phi_{2,1,}^{z}
\end{array}\right]}
\end{aligned}
$$

and

$$
\left\{p_{B}^{R}\right\}=\left\{\begin{array}{c}
p_{a}^{B} \\
p_{\ell}^{B} \\
p_{r}^{B}
\end{array}\right\}, \quad\left\{p_{B}^{N}\right\}=\left\{\begin{array}{c}
p_{1}^{B} \\
1 \\
1 \\
1 \\
p_{n}^{B}
\end{array}\right\}, \quad\left\{p_{0}^{B}\right\}=\left\{\begin{array}{c}
p_{a}^{0} \\
p_{l}^{0} \\
p_{r}^{0}
\end{array}\right\}, \quad\left\{p_{0}^{N}\right\}=\left\{\begin{array}{c}
p_{1}^{0} \\
1 \\
1 \\
1 \\
p_{a}^{0}
\end{array}\right\}
$$

Due to the fact that there exist three equations of compatibility the components in the $\{p\}$ vector are not independent. Recall that if there are $\ell$ variables and there exists $k$ equations of constraint between them, there will be $i=l-k$ independent variables or degrees-of-freedom. In our case we have $m+n+6$ variables and three equations of constraint so that the number of degrees-of-freedom is $m+n+3$. It is entirely arbitrary as to which variables are selected as the dependent variables but in this study we choose the Booster generalized coordinates $\mathrm{p}_{a}^{B}, \mathrm{p}_{\ell}^{\mathrm{B}}$, $\mathrm{p}_{\mathrm{r}}^{\mathrm{B}}$. Equation (83) may be written as

$$
\left[\begin{array}{l:l}
\hat{\phi}_{B}^{R} & A
\end{array}\right]\left\{\begin{array}{c}
p_{B}^{R} \\
\hdashline q
\end{array}\right\}=0
$$

from which 


$$
\left[\hat{\phi}_{B}^{R}\right]\left\{p_{B}^{R}\right\}+[A]\{q\}=0
$$

where $\{q\}$ is now a column vector of independent generalized coordinates. The dependent coordinates may now be obtained from Equation (86) as

$$
\left\{p_{B}^{R}\right\}=-\left[\hat{\phi}_{B}^{R}\right]^{-1}[A]\{q\}
$$

From this we derive an equation which relates the complete $\{\mathrm{p}\}$ vector to the independent $\{q\}$ sub-vector

$$
\{p\}=\left\{\begin{array}{c}
p_{B}^{R} \\
\hdashline q
\end{array}\right\}=\left[\begin{array}{c}
-\left[\hat{\phi}_{B}^{R}\right]^{-1}[A] \\
\hdashline[I]
\end{array}\right]\{q\}
$$

The rectangular matrix in this equation is of order $(m+n+6) \times(m+n+3)$ and [I] is an identity matrix of order $m+n+3$. Equation (88) may be written briefly as

$$
\{p\}=[\beta]_{p}\{q\}
$$

where $[\beta]_{p}$ is a coupling matrix analogous to the one defined by Hurty ${ }^{(2)}$ with the subscript $p$ indicating that the pin-slip component modes were used for this analysis.

For the synthesis of the free-free-component modes recall that all the displacements are referenced with respect to the interior nodes. Therefore, due to this and the fact that boundary nodes in the free-free component configurations have non-zero displacements we must first determine the boundary displacements in terms of the free-free rigidbody and elastic modes. Note that for this case the elastic components are not all zero as was the case for the pin-slip configurations. The displacement compatibility Equations ( $8 \mathrm{la}, \mathrm{b})$ still apply here but we must make the following additional requirements in order to transform the dis placements at the interior nodes to the boundary nodes

$$
\begin{aligned}
& \mathrm{x}_{1,2}=\mathrm{x}_{2}+\delta_{1} \theta_{2} \\
& \mathrm{x}_{1,2}=\mathrm{x}_{8}-\delta_{1} \theta_{8} \\
& \mathrm{z}_{1,1}=\mathrm{z}_{2}, \quad \mathrm{z}_{1,2}=\mathrm{z}_{8}, \quad \mathrm{z}_{2,1}=\mathrm{z}_{5}, \mathrm{z}_{2,2}=\mathrm{z}_{11}
\end{aligned}
$$

The displacements $\mathbf{x}_{2}, \theta_{2}, \mathbf{x}_{8}$, and $\theta_{8}$ may be written as 


$$
\begin{aligned}
& x_{2}=\phi_{2, a}^{x} p_{a}^{0}+\sum_{j=1}^{m} \phi_{2, j}^{x} p_{j}^{0} \\
& \theta_{2}=\phi_{2, s}^{\theta} p_{r}^{0}+\sum_{j=1}^{m} \phi_{2, j}^{\theta} p_{j}^{0} \\
& x_{8}=\phi_{8, a}^{x} p_{a}^{B}+\sum_{j=1}^{n} \phi_{8, j}^{x} p_{j}^{8} \\
& \theta_{8}=\phi_{8, s}^{\theta} p_{r}^{\theta}+\sum_{j=1}^{n} \phi_{8, j}^{\theta} p_{j}^{\theta}
\end{aligned}
$$

where $\phi_{2, a}^{x}$ is the normalized displacement of $x_{2}$ due to rigid-body axial modes, etc. Similarly, the displacements given by Equation (90c) may be written as a combination of rigid-body and elastic modal displacements. Using Equations ( $91 \mathrm{a}, \mathrm{b}, \mathrm{c}, \mathrm{d})$ and Equations $(90 \mathrm{a}, \mathrm{b}, \mathrm{c})$ and substituting into Equations $(81 \mathrm{a}, \mathrm{b})$ we arrive at a coupling matrix for the free-free component analysis which again relates the complete $\{p\}$ vector to the independent $\{q\}$ sub-vector by

$$
\{p\}=[\beta],\{q\}
$$

with the subscript $f$ indicating that the free-free component modes are used in this analysis. Having obtained the coupling matrices which enable us to connect the Booster and Orbiter components to form the System we now proceed to the System eigenvalue problem.

3. Eigenvalue Problem. As stated in the previous section, the coupling matrix $[\beta]$ relates the complete $\{p\}$ vector to the independent sub-vector $\{q\}$ which enables us to obtain the coupled System equations. Substituting either Equation (89) or Equation (92) (depending on whether we are synthesizing with the pin-slip component modes or the free-free component modes) into Equation (79), and then premultiplying by the transpose of the appropriate coupling matrix, we obtain the System equations of motion

$$
[m]_{s}\{\ddot{q}\}+[c]_{s}\{\dot{q}\}+[K]_{s}\{q\}=\{Q(t)\}_{s}
$$

where 


$$
\begin{aligned}
& {[M]_{s}=[\beta]^{\top}[M][\beta]} \\
& {[C]_{s}=[\beta]^{\top}[C][\beta]} \\
& {[K]_{s}=[\beta]^{\top}[K][\beta]} \\
& {[Q(t)]_{s}=[\beta]^{\top}\{\mathcal{F}(t)\}}
\end{aligned}
$$

Equation (93) is an $(m+n+3) \times(m+n+3)$ matrix equation where again $\mathrm{m}=$ number of Orbiter component modes and $\mathrm{n}=$ number of Booster component modes used in the analysis. To determine the frequencies and mode shapes for the System we solve [using Equation (93)] the undamped eigenvalue problem

$$
\left|\omega^{2}[I]-[D]\right|=0
$$

where

$$
[D]=[m]_{s}^{-1}[K]_{s}
$$

A modified Jacobi method was used to obtain the eigenvalues and eigenvectors of Equation (95). The eigenvectors are normalized and may be written as an $m+n+3$ square matrix in the following form

$$
[\gamma]=\left[\left\{\gamma_{1}\right\},\left\{\gamma_{2}\right\}, \ldots\left\{\gamma_{m+n+3}\right\}\right]
$$

These eigenvectors may be used to relate the generalized coordinate vector $\{q\}$ to a new vector $\{\eta\}$ which expresses displacements in a system of normal coordinates:

$$
\{q\}=[\gamma][\eta]
$$

At this point, however, we do not solve for the forced responses in the $\{\eta\}$ coordinate system. It is more useful to make a transformation back to our original coordinate system $\{\bar{u}\}$. The reason for this transformation at this point is twofold. First, it permits us to compare directly the analytically predicted mode shapes and forced responses with the responses measured in the laboratory. Second, and most importantly, it permits us to apply a newly-developed method for synthesizing the component damping which appears to be extremely promising in terms of the accuracy obtained. This method of damping synthesis is discussed in detail in Section VI of this report. At this point we will only say that the method permits us to obtain System modal damping which results in a diagonal System damping matrix. This causes the equations of motion to be uncoupled and permits rapid solution of the forced responses. 
For our 39 coordinate model ( 21 for the Booster and 18 for the Orbiter) we may obtain the System normalized mode shapes in the $\{\hat{u}\}$ coordinate system from the following equation

$$
[\hat{\phi}]_{S} \rightarrow\left[\begin{array}{c:c}
\phi_{\theta} & 0 \\
\hdashline 0 & \phi_{0}
\end{array}\right]_{p, \rho}[\beta]_{p, \rho}[\gamma]_{p, \rho}
$$

where again the subscript $p$ denotes the pin-slip case and $f$ denotes the free-free case. The System normalized modes are obtained by dividing each column of the resulting matrix product given in the above equation by an appropriate constant. The submatrices $\left[\phi_{B}\right]$ and $\left[\phi_{0}\right]$ are the component Booster and Orbiter modes used in the analysis, $[\beta]$ is the coupling matrix, and $[\gamma]$ is the eigenvector matrix given by Equation (97). The sizes of the matrices at this point are as follows:

$$
\begin{array}{ccc}
{[\hat{\phi}]_{s}} & : & 39 \times(\mathrm{n}+\mathrm{m}+3) \\
{\left[\begin{array}{c:c}
\phi_{8} & \vdots \\
\hdashline & \phi_{0}
\end{array}\right]} & : & 39 \times(\mathrm{n}+\mathrm{m}+6) \\
{[\beta]} & : & (\mathrm{n}+\mathrm{m}+6) \times(\mathrm{n}+\mathrm{m}+3) \\
{[\gamma]} & : & (\mathrm{n}+\mathrm{m}+3) \times(\mathrm{n}+\mathrm{m}+3)
\end{array}
$$

Note that the System mode shapes $[\phi]$, are given in the $\{\hat{u}\}$ coordinate system which includes displacements at both interior and interface point positions. For convenience, we finally transform these mode shapes to the $\{\overline{\mathrm{u}}\}$ or interior coordinates by means of Equation (21). That is, for the $j$-th column or mode shape we have

$$
\left\{\bar{\phi}_{j}\right\}_{s}=[\alpha]\left\{\hat{\phi}_{j}\right\}_{s}
$$

The displacements $\left(x_{1}, z_{1}, \theta_{1}\right)$ at node $i$ may now be expressed as a summation of system modes and system generalized coordinates, i.e.,

$$
\begin{aligned}
& \mathrm{x}_{1}=\phi_{1, \mathrm{a}}^{\mathrm{x}} \mathrm{p}_{\mathrm{a}}^{\mathrm{s}}+\phi_{1,2}^{\mathrm{x}} \mathrm{p}_{l}^{\mathrm{s}}+\phi_{1, \mathrm{r}}^{\mathrm{x}} \mathrm{p}_{\mathrm{r}}^{\mathrm{s}}+\sum_{\mathrm{k}=1}^{\mathrm{n}+\mathrm{m}} \phi_{1, \mathrm{k}}^{\mathrm{x}} \mathrm{p}_{\mathrm{k}}^{\mathrm{s}} \\
& \mathrm{z}_{1}=\phi_{1, \mathrm{a}}^{2} \mathrm{p}_{\mathrm{a}}^{\mathrm{s}}+\phi_{1, l}^{2} \mathrm{p}_{\ell}^{\mathrm{s}}+\phi_{1, \mathrm{r}}^{2} \mathrm{p}_{\mathrm{r}}^{\mathrm{s}}+\sum_{\mathrm{k}=1}^{\mathrm{n}+\mathrm{m}} \phi_{1, \mathrm{k}}^{2} \mathrm{p}_{\mathrm{k}}^{\mathrm{s}} \\
& \theta_{1}=\phi_{1, \mathrm{a}}^{\prime} \mathrm{p}_{\mathrm{a}}^{\mathrm{s}}+\phi_{1,2 \mathrm{p} \ell}^{\theta}+\phi_{1, \mathrm{r}}^{\theta} \mathrm{p}_{\mathrm{r}}^{\mathrm{s}}+\sum_{\mathrm{k}=1}^{\mathrm{n}+\mathrm{m}} \phi_{1, \mathrm{k}}^{\theta} \mathrm{p}_{\mathrm{k}}^{\mathrm{s}}
\end{aligned}
$$


or letting

$$
\left\{\bar{u}_{1}\right\}_{s}=\left\{\begin{array}{l}
x_{1} \\
z_{1} \\
\theta_{1}
\end{array}\right\}_{s}
$$

we may write Equations $(100 a, b, c)$ in matrix form as

$$
\left\{\bar{u}_{1}\right\}=\left[\begin{array}{l:l}
\bar{\phi}_{1}^{R} & \bar{\phi}_{1}^{N}
\end{array}\right]_{S}\left\{\begin{array}{c}
p_{1}^{R} \\
\hdashline p_{1}^{N}
\end{array}\right\}_{S}
$$

For all the nodes on both the Booster and the Orbiter we may combine and arrange the displacements in the following manner

$$
\{\bar{u}\}_{S}=\left\{\begin{array}{l}
\{\bar{u}\}_{B} \\
\{\bar{u}\}_{0}
\end{array}\right\}
$$

where

$$
\{\bar{u}\}_{B}=\left\{\begin{array}{c}
\bar{u}_{7} \\
\bar{u}_{8} \\
\vdots \\
\bar{u}_{13}
\end{array}\right\} \quad \text { and } \quad\{\bar{u}\}_{0}=\left\{\begin{array}{c}
\bar{u}_{1} \\
\bar{u}_{2} \\
\vdots \\
\bar{u}_{6}
\end{array}\right\}
$$

which are synonomous with Equation (8). Likewise, Equation (102) may be written to include all the nodes as follows:

$$
\{\bar{u}\}_{S}=\left[\begin{array}{c:c}
\bar{\phi}_{B}^{R} & \bar{\phi}_{B}^{N} \\
\hdashline \bar{\phi}_{0}^{R} & \bar{\phi}_{0}^{N}
\end{array}\right]\left\{\begin{array}{l}
p^{R} \\
\hdashline p^{N}
\end{array}\right\}=[\bar{\phi}]_{S}\{p\}_{S}
$$

where the first 21 rows of the modal matrix correspond to Booster modal diaplacements and the next 18 rows correspond to Orbiter modal displacements with each mode in the matrix arranged column-wise. The generalized System mass and stiffness matrices may now be written as

$$
\begin{aligned}
& {[\tilde{M}]_{S}=\left[\begin{array}{c:c}
\bar{\phi}_{B}^{R} & \bar{\phi}_{B}^{N} \\
\hdashline \bar{\phi}_{0}^{R} & \bar{\phi}_{0}^{N}
\end{array}\right]^{\top}\left[\begin{array}{c:c}
\overline{\mathrm{M}}_{B} & 0 \\
\hdashline 0 & \overline{\mathrm{M}}_{0}
\end{array}\right]\left[\begin{array}{c:c}
\bar{\phi}_{B}^{R} & \bar{\phi}_{B}^{N} \\
\hdashline \bar{\phi}_{0}^{R} & \bar{\phi}_{0}^{N}
\end{array}\right]} \\
& {[\tilde{K}]_{S}=\left[\begin{array}{c:c}
\bar{\phi}_{B}^{R} & \bar{\phi}_{B}^{N} \\
\hdashline \bar{\phi}_{0}^{R} & \bar{\phi}_{0}^{N}
\end{array}\right]^{\top}\left[\begin{array}{c:c}
\overline{\mathrm{K}}_{B} & 0 \\
\hdashline 0 & \overline{\mathrm{K}}_{0}
\end{array}\right]\left[\begin{array}{c:c}
\bar{\phi}_{B}^{R} & \bar{\phi}_{B}^{N} \\
\hdashline \bar{\phi}_{0}^{R} & \bar{\phi}_{0}^{N}
\end{array}\right]}
\end{aligned}
$$


where the tilde $(\sim)$ indicates that these quantities are with respect to the System normal modes.

4. Solution of Coupled Equations. Equation (93) represents the System equations of motion in matrix form and at this point is highly coupled. Solution of this system of coupled, damped equations would appear to be a straightforward possible method of damping prediction for the System. It was decided to pursue this possibility by solving for System modal damping. A check on the damping matrix was first performed by solving the damped eigenvalue problem. A solution to the undamped eigenvalue problem showed very good agreement with the natural frequencies and mode shapes obtained on the model in the laboratory. A method for solving equations of motion for a damped linear system was developed by Foss (7). The procedure is fairly lengthy and only the salient features of the method will be discussed here.

For a damped system having $n$ equations of motion, the eigenvalue and eigenvectors are complex for a system that is less than critically damped. This means that $2 \mathrm{n}$ equations are required since two items of information (magnitude and phase angle) are required to determine each mode. Following Foss' method we write the following matrix identity

$$
[m]_{s}\{\dot{q}\}-[m]_{s}\{\dot{q}\}=\{0\}
$$

where $[m]_{s}$ is the mass matrix shown in Equation (93). This equation is combined with Equation (93) which is repeated here

$$
[m]_{s}\{\ddot{q}\}+[C]_{s}\{\dot{q}\}+[K]_{s}\{q\}=\{Q(t)\}_{s}
$$

The two equations are then combined as follows:

$$
[S]\{\dot{y}\}+[T]\{y\}=\{Y(t)\}
$$

where

$$
\begin{aligned}
& {[\mathrm{S}]=\left[\begin{array}{c:c}
0 & m_{\mathrm{s}} \\
\hdashline m_{\mathrm{s}} & c_{\mathrm{s}}
\end{array}\right]} \\
& {[\mathrm{T}]=\left[\begin{array}{c:c}
m_{\mathrm{s}} & 0 \\
\hdashline 0 & x_{\mathrm{s}}
\end{array}\right]} \\
& \{\mathrm{y}\}=\left\{\begin{array}{c}
\dot{\mathrm{q}} \\
\hdashline \mathrm{q}
\end{array}\right\}
\end{aligned}
$$




$$
\{Y(t)\}=\left\{\begin{array}{c}
0 \\
-Q(t)_{3}
\end{array}\right\}
$$

The solution to the homogeneous part of Equation (108) is obtained by setting the right-hand side equal to zero and may be expressed in the exponential form, $e^{\text {pt. }}$. Therefore, we have the following relationship:

$$
\{\dot{\mathrm{y}}\}=\mathrm{p}\{\mathrm{y}\}
$$

which when substituted into Equation (108) gives

$$
\mathrm{p}[\mathrm{S}]\{\mathrm{y}\}=-[\mathrm{T}]\{\mathrm{y}\}
$$

Equation (111) is an eigenvalue problem that yields a set of $2 \mathrm{n}$ eigenvalues and a set of $2 n$ eigenvectors. The imaginary parts of the eigenvalues give the frequencies of the free vibrations and the real parts give a measure of the rate of exponential decay of the amplitudes of free vibration. The eigenvalues may be written as follows:

$$
p_{\jmath}=-\zeta_{\jmath} \omega_{\jmath}^{d} \pm i \omega_{\jmath}^{d}
$$

where $w_{j}^{d}$ is the damped natural frequency for the $j$-th mode and $\zeta_{j}$ is the damping factor for the $j$-th mode which can be determined by dividing the real part of $p_{j}$ by $\omega_{j}^{\mathrm{d}}$. It should be noted that $\zeta_{j}$, as determined from the complex eigenvalue procedure, is identical to that which should be expected to result if the coupled forced response were solved directly, and $\zeta_{f}$ were determined from a $\frac{1}{2}$-bandwidth procedure applied at each resonant frequency.

Comparison of the damping factors obtained by solving the above eigenvalue problem with those obtained on the model showed the results to be extremely poor with the majority of errors being in the neighborhood of 100-325 percent, as will be shown in Section VII. B. As a result of the poor agreement, solution of the nonhomogeneous equations would have produced equally poor results especially at the System natural frequencies, since here the responses are damping controlled. Anticipating this fact at the outset of this program, we studied various other ways by which the System damping could be synthesized from the component damping with favorable results. A new method for damping synthesis was developed which produced extremely good results, at least for our present fairly simple model. Details of this method will be given in Section VI. However, we first conclude the theoretical development by indicating how predicted modal damping is to be used for predicting forced response. 
5. System Forced Response Using Predicted Modal Damping.

Using the modal mass and stiffness matrices given by Equations (106a, b), we now introduce a modal damping matrix [ $[\tilde{C}]_{s}$ which is obtained from the procedures outlined in Section VI of this report. This damping matrix is diagonal with the $j$-th element given by $\tilde{c}_{j}=2 \tilde{m}_{j} \omega_{j} \delta_{j}$ where $\omega_{j}$ is the $j$-th natural frequency obtained from the eigenvalue problem given by Equation (95) and $S_{j}$ is the predicted damping factor for the $j$-th System mode. The uncoupled equations of motion are now written in matrix form as

$$
[\tilde{m}]_{s}\{\ddot{p}\}_{s}+[\tilde{\mathcal{C}}]_{s}\{\dot{p}\}_{s}+[\tilde{K}]_{s}\{p\}_{s}=\{\tilde{Q}(t)\}_{s}
$$

where $\{\tilde{Q}(t)\}_{S}$ is a vector of generalized applied forces. Procedures for obtaining the System forced responses are the same as for the component forced responses which were given in Section III.C. It should be noted that the damping factors used in the System forced response analyses are the predicted ones and not those obtained experimentally, although a comparison of the two is given in a later section. Once the generalized $\{p\}$ coordinates are known, the displacements may be obtained by substituting into Equation (105). 


\section{EXPERIMENTAL PROCEDURES}

\section{A. Background Discussion}

In order to utilize damping results from component tests for prediction of System behavior, it was recognized that some form of correlation of component results would first have to be established. Several methods of doing so were investigated. The method finally found to work best is based on a modification of a damping energy correlation which was presented in Reference 1. However, we now will show that the present modification is necessary in order for the correlation to be valid.

A straightforward dimensional analysis of the component damping results can be performed by considering the following variables:

$$
\begin{aligned}
& D_{0}=\text { Damping energy dissipated per cycle } \\
& M=\text { Modal mass } \\
& \omega=\text { Natural frequency of a given mode } \\
& \mathbf{x}_{0}=\begin{array}{l}
\text { Peak modal amplitude for steady-state sinusoidal } \\
\text { vibran }
\end{array} \\
& l=\text { Some characteristic length }
\end{aligned}
$$

A dimensional analysis results in the nondimensional equation

$$
\frac{\mathrm{D}_{c}}{\mathrm{M} \omega^{2} \mathrm{x}_{0}^{2}}=\mathscr{F}_{1}\left(\mathrm{x}_{0} / \ell\right) \quad \text { or } \quad \frac{\mathrm{D}_{c}}{\mathrm{~T}_{0}}=\mathscr{F}_{1}\left(\mathrm{x}_{0} / \ell\right)
$$

where $T_{0}=\frac{1}{2} M \omega^{2} x_{0}^{2}$ is the peak steady state kinetic energy at amplitude $x_{0}$. This equation can be written in dimensional form as

$$
D_{c}=F_{2}\left(T_{0}, x_{0} / \ell\right)
$$

This result shows that damping energy is a function of two independent variables, not just kinetic energy alone, as was originally assumed in Reference i.

In view of Equation (114), single-curve correlation will result only if $x_{0} / l$ is held constant, and a family of curves is obtained, one for each amplitude. Sample results for one Booster configuration are 
shown in Figure 5, plotted in the form of Equation (114). Strictly speaking, the most general procedure for obtaining such data is to measure input energy at each of the component resonances, while holding the amplitude constant. Kinetic energy is computed from measured frequencies and theoretical modal mass. However, for a system with many resonances, it is extremely difficult to obtain accurate measurements at low frequencies, for those amplitudes which are appropriate at higher modes. On the other hand, if the damping forces are found to vary essentially linearly with amplitude $x_{0}$, and damping energy linearly with $x_{0}^{2}$, then measurements can be made at any convenient amplitude, and adjusted to the values corresponding to a given value of $x_{0} / l$. Further, if this condition does exist, then it is also possible to measure a modal damping ratio $\zeta$ by means of conventional free decay or half-bandwidth techniques, and the damping energy can be calculated from

$$
D_{c}=4 \pi T_{0} S
$$

The latter procedure was utilized in reducing the majority of the data in the present program. However, it should be noted emphatically, that data such as that shown in Figure 5 can be generated for any form of damping process (including one nonlinear with amplitude), so long as damping energy is measured directly, and amplitudes are either held constant, or values are extrapolated by some suitable procedure.

There is another extremely important point which must be recognized with regard to the form of plot displayed in Figure 5. That is, the points represent measurements made only at resonant conditions for a given component. When a curve is drawn through these points, we automatically imply that damping energy can be obtained at intermediate, off-resonant kinetic energies as well from these curves. In other words, since we have a system with light damping and reasonably separated modes, Figure 5 is not only a plot of modal damping energy versus peak modal kinetic energy, but it can be hypothesized to be a plot of total damping energy versus total peak kinetic energy as well, since at resonance, only one mode dominates. The latter statement must hold true regardless of the vibrational form of the component. It would be appropriate to consider measurements at off-resonant conditions to obtain further data to support this assertion, since it will be a fundamental hypothesis to be used in the later development of our method for prediction of System modal damping. However, measurements at such conditions are usually highly inaccurate, and other further evidence which confirms this hypothesis will be given near the end of the report. 


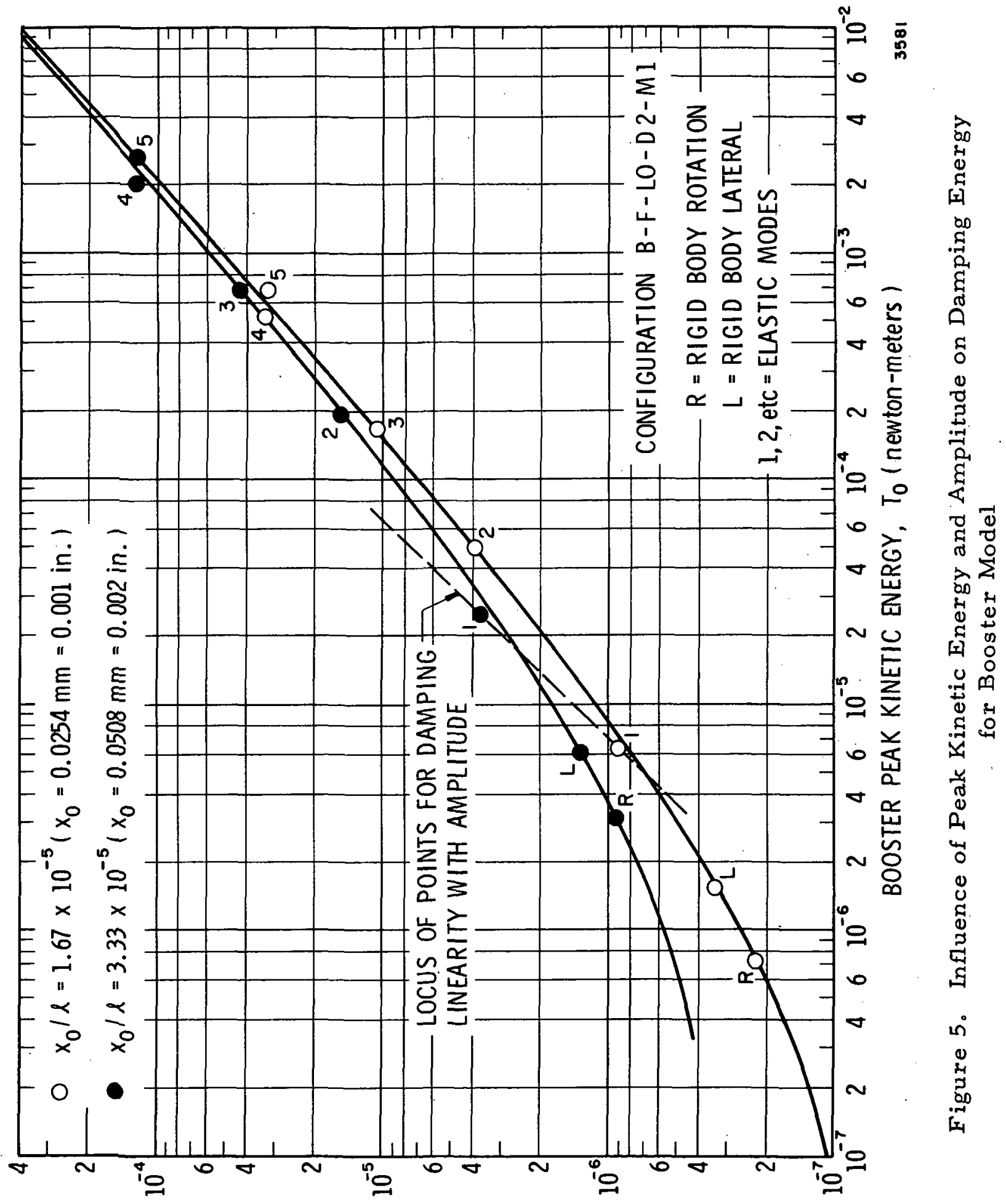

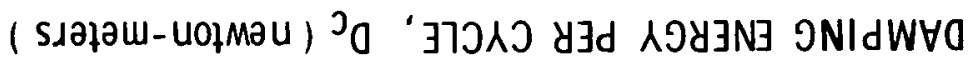




\section{B. General Procedures}

It is recognized that the primary emphasis of this program is to develop a method for prediction of System damping. However, in order to do so, use of other dynamic modal properties of the components and the System must be included. It is logical that natural frequencies and mode shapes must be considered as part of the comparison of results from analytical and experimental models. Likewise, comparison of results for steady-state forced response are also desirable in addition to values for modal damping. Therefore, we will include data to show the effects of a variety of parameters on component behavior, and in many cases will also include predicted values for the components for comparison. These results will establish a confidence in the accuracy of the component mathematical models. We subsequently will then discuss results for the System.

The approximate order of conducting the experiments has been indicated in Table $I(a)$. Once the apparatus for a given configuration was set up, natural frequencies and mode shapes were measured first. Excitation was provided by a very light electrodynamic exciter which was located at Mass 10 on the Booster and at Mass 4 on the Orbiter. For System configurations part of the data were obtained by driving on each component. Mode shapes were measured in the lateral directions only by means of multiple piezoelectric accelerometers which were mounted to various masses. Most of the accelerometer cables were removed for subsequent damping measurements, since it was found that motion in these cables could cause damping of the same order as the very light structural damping exhibited by the components when tested in air.

Damping measurements were performed by the 1/2-bandwidth technique at first. Only slight nonlinearity with amplitude was usually encountered. However, those configurations involving the lightest damping could best be tested using the free decay method. Most of the data were subsequently obtained using this method, since a permanent record resulted, and the results were commensurate with those obtained from 1/2-bandwidth measurements.

During this entire program, careful measures were taken to ensure the reliability of the results. Oil temperatures and viscosities were monitored several times daily. Independent calibration of the force exciter and accelerometers were repeated on several occasions. Corrections to damping values to allow for viscosity changes were based on day-to-day plots of the data. Tests on various modes were repeated at later times when possible in order to provide cross-checks in the data. Sample data were often taken at various amplitudes to spot any significant nonlinearity. After all these measures, it is estimated that the worst 
error for modal damping measurements is $\pm 15 \%$, although most of the data is much less. This is not bad, however, as damping measurements on typical structures are usually much worse. It is also surmized that only the very first few test configurations experienced much of the worst error, as improvements in procedures were incorporated as time progressed and behavior of the apparatus was better established.

Tests for properties in rigid-body modes were necessary to provide data for System damping prediction. This was a consequence solely of our particular design of providing damping; that is, damping relative to the fixed ground. Axial and lateral tests were conducted by measuring decay rates at the low frequencies (about $1 \mathrm{~Hz}$ ) of the models on the simulated free-free suspension system. Rotational tests were conducted at about the same frequency by supporting the models on a knife-edge at their CG, and stabilizing the model with a small lateral spring. In all cases, the same oil levels were maintained as for the tests for elastic modes.

\section{Link Damper Calibration}

The link damping mechanism described in Figure 3 evolved from a series of other mechanisms which proved to be far more unreliable. It would have been desirable to have calibrated this device with a completely independent apparatus. However, in order to save time, it was decided to calibrate it directly in place on the combined System. It must be emphasized that this in no way deters from the validity of the results that followed.

For calibration, the link damper was. installed on the combined System, while it was supported in air (i.e., in the DO condition with no oil in the reservoirs). Relative velocity across the link was monitored at a steady-state forced response for each mode, first in the Ll condition (with no feedback in the damper). Measurement of the input energy thus allows establishing a small damping energy reference level associated with the internal structural stresses and the motion of the flexure pins. This level was usually less than $0.1 \%$ critical in any mode. Then, the energy level with feedback on was also measured at the same input amplitude. The difference of the two energy levels was that added by the link damper, and was reduced to an equivalent viscous damping coefficient, since the measured relative velocity across the link was available in all cases. This procedure resulted in the damping coefficients listed in Table III. A further check during these procedures showed that no significant nonlinearity or change in mode shapes occurred for the various levels of damping employed. This was somewhat surprising, since at the largest damping level (L4), the joint provided more than 30 times the damping energy absorbed in the rest of the structure when it was supported in air. 
TABLE III. LINK DAMPING COEFFICIENTS FOR L2, L3, L4

\begin{tabular}{|c|c|c|c|}
\hline Mode No. & $C^{(1)}$ & $C^{(a)}$ & $C^{(3)}$ \\
\hline 1 & 93.05 & 185.83 & 354.63 \\
\hline 2 & 108.80 & 212.78 & 523.43 \\
\hline 3 & 122.98 & 235.47 & 434.07 \\
\hline 4 & 77.59 & 161.71 & 374.49 \\
\hline 5 & 123.13 & 270.94 & 509.25 \\
\hline 6 & 113.20 & 178.73 & 431.23 \\
\hline 7 & -- & -- & $\ldots$ \\
\hline 8 & $\ldots$ & $\ldots$ & $\ldots$ \\
\hline 9 & 91.92 & 270.94 & 187.24 \\
\hline 10 & $\ldots$ & - & $\ldots$ \\
\hline
\end{tabular}
(1) L2 Link Damping
Units for C are newton-sec/meter
(2) L3 Link Damping
(3) L4 Link Damping 


\section{COMPONENT RESULTS}

\section{A. Pin-Slip Configuration}

1. Natural Modes. The experimental and predicted normalized mode shapes for the Booster in the $M 1$ and $M 2$ mass configurations are shown in Figures $6 \mathrm{a}$ and $6 \mathrm{~b}$, respectively. Only the lateral direction is presented although longitudinal and rotational motions were also present through coupling. Six mode shapes were determined experimentally for each mass configuration. For the Orbiter in the M1 and M2 mass configurations, the normalized mode shapes are shown in Figures $7 \mathrm{a}$ and $7 \mathrm{~b}$, respectively. All the experimental mode shapes are shown in the D2 damping condition. Due to the slight difference in densities of the various oils used to produce the damping, it was surmised that there might be a change in mode shapes between damping conditions. Comparison of the mode shapes among the various damping conditions showed, however, that any changes were insignificant. The experimental and theoretical natural frequencies and modal masses are presented in Table IV(a) for the Booster and in Table IV(b) for the Orbiter. Results are presented for various damping conditions to show the effect of the various oil densities on the natural frequencies of the component. The theoretical frequencies given were obtained for the D2 oil density condition.

It was discovered during the course of experimentation that there was a small amount of translational compliance at the pin-joints. This seemed to affect particularly both the Booster and Orbiter fourth modes, with the difference in theoretical and experimental frequencies being about 7 to 8 percent. The percent difference is based on experimental frequencies. This difference was not considered exceptionally bad, and it was further surmised that this discrepancy would not influence damping results. Therefore, no modifications were made to the pin-joints.

2. Damping. Experimental damping energy per cycle versus kinetic energy curves are shown in Figures $8 \mathrm{a}$ and $8 \mathrm{~b}$ for the Booster and Orbiter substructures, respectively. The curves were obtained by fairing through the experimental data points given by the symbols, as has already been discussed in Section IV. A. A least squares fit would have been appropriate but was not considered here. Results are shown for the D0, D2, and D3 damping conditions in the M1 mass configuration and for the D2 damping condition in the M2 mass configuration. It should be noted that both the M1 and M2 mass configuration energy curves appear to be the same (at least within the scatter of the data) for the D2 condition. This result was particularly encouraging since it seems to indicate that for different mass-configured substructures with similar 


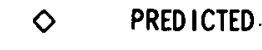

EXPERIMENTAL
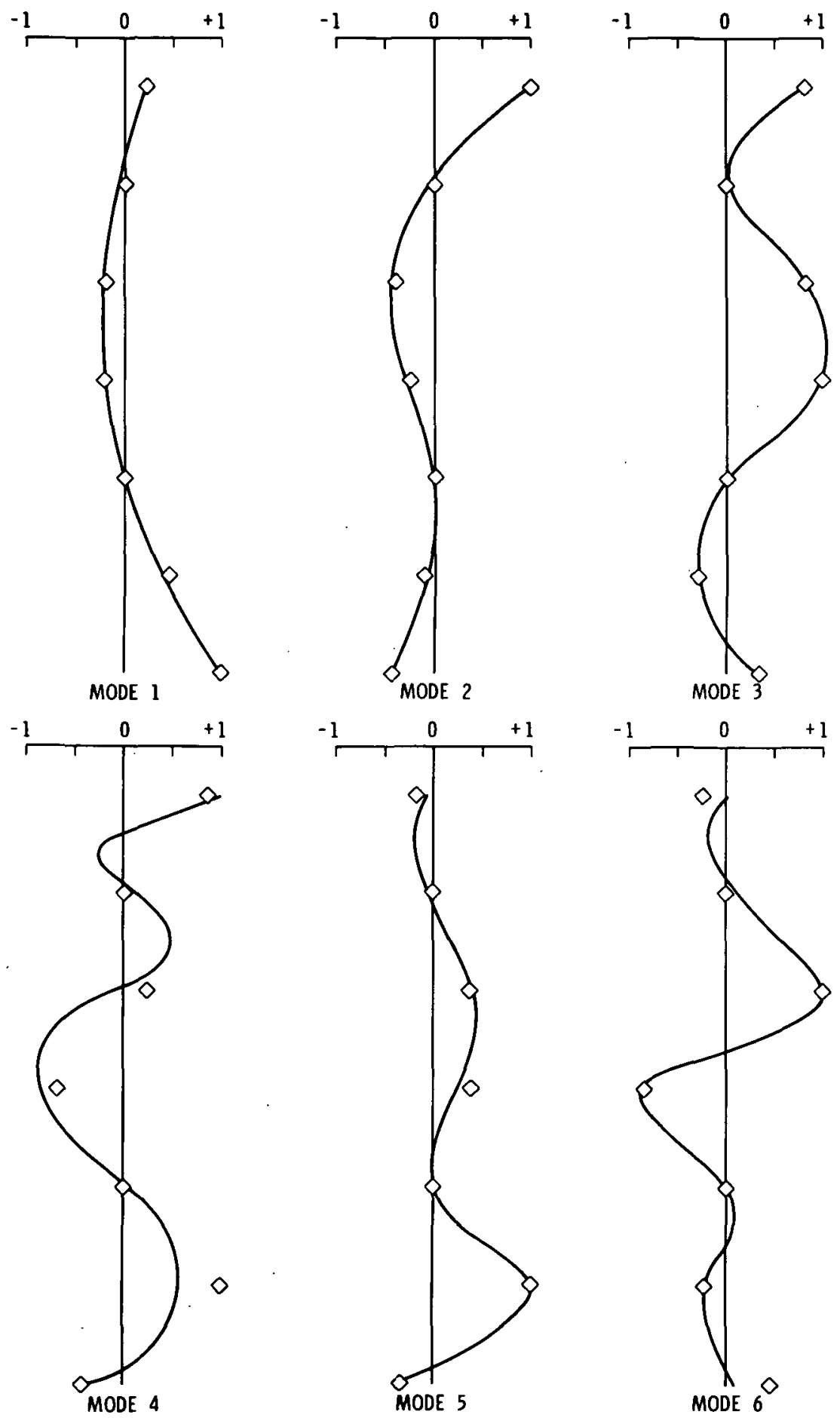

Figure 6. Booster Mode Shapes for Pin-Slip Configurations (a) MI Mass Condition 


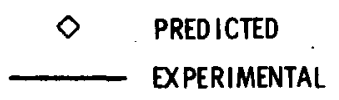

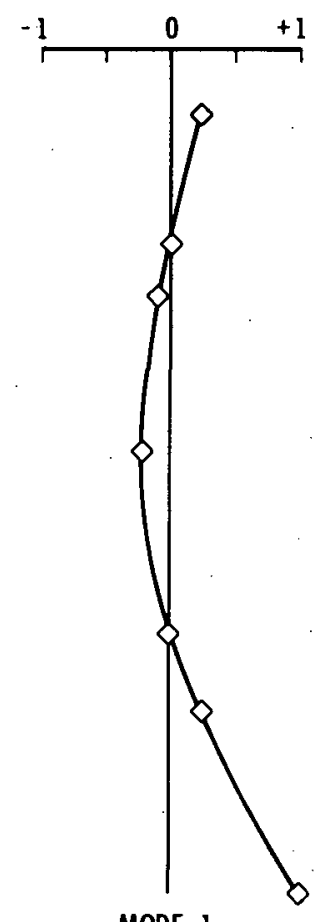

MODE 1

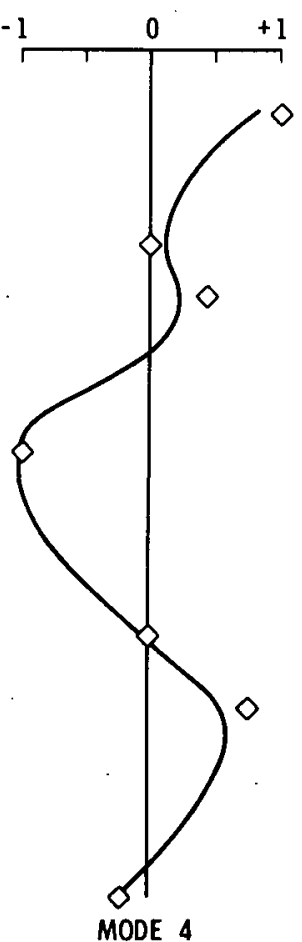

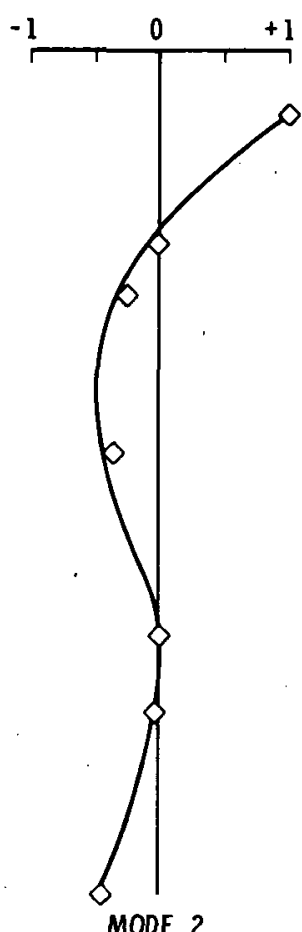

MODE 2
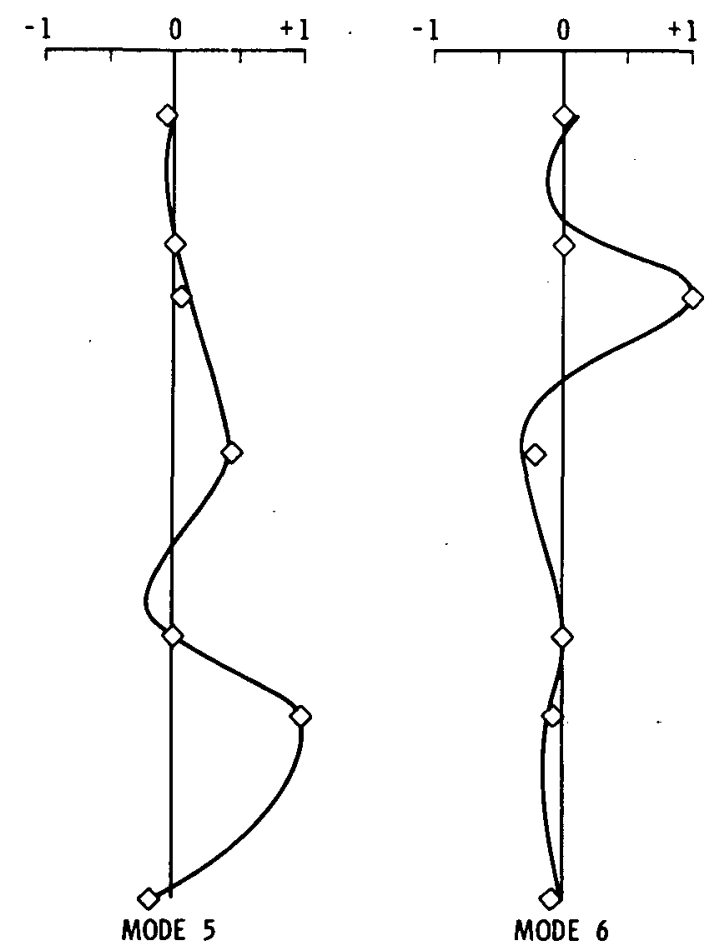

Figure 6. Booster Mode Shapes for Pin-Slip Configurations (b) M2 Mass Condition 

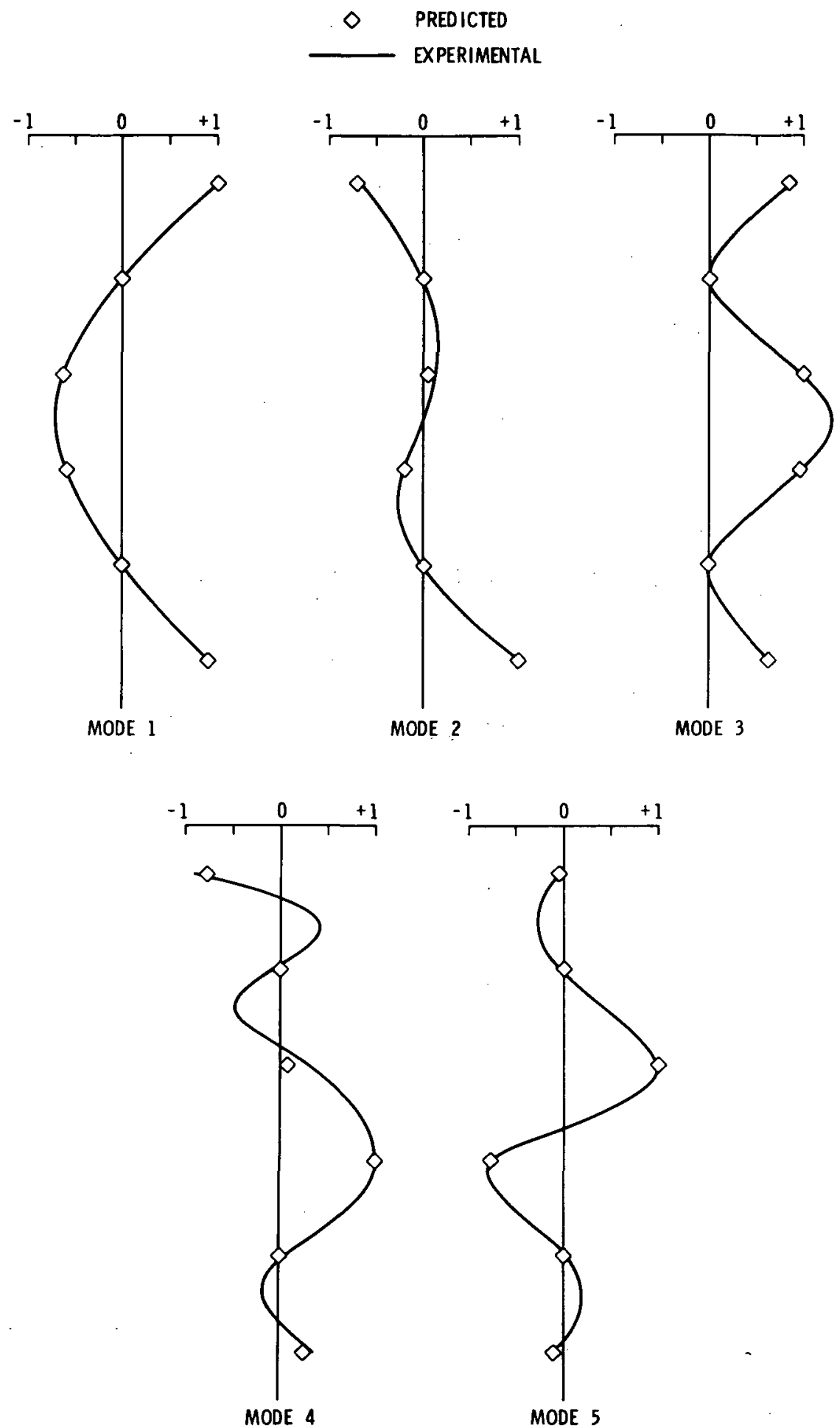

Figure 7. Orbiter Mode Shapes for Pin-Slip Configurations (a) Ml Mass Condition 


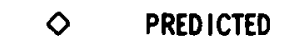

EXPERIMENTAL

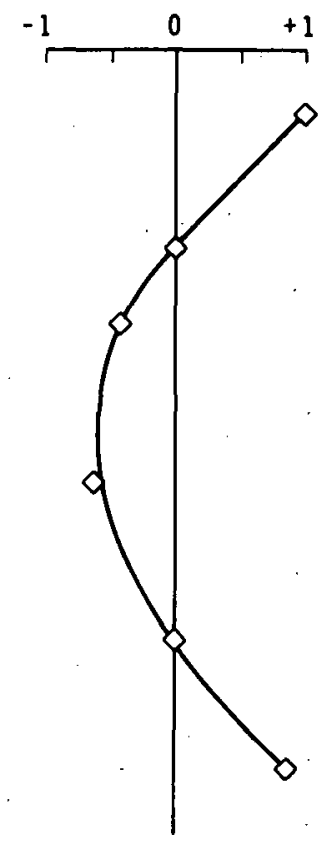

MODE 1

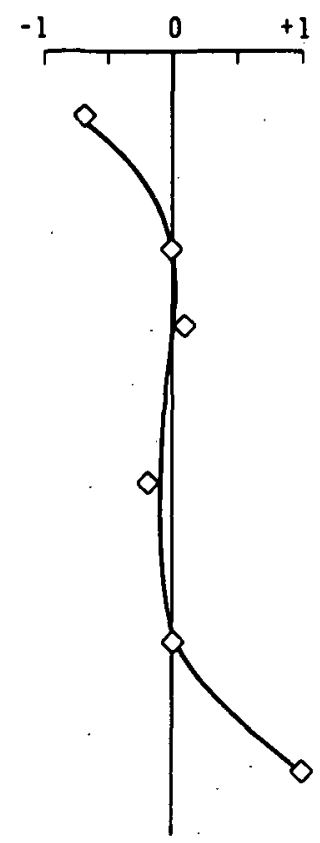

MODE ?

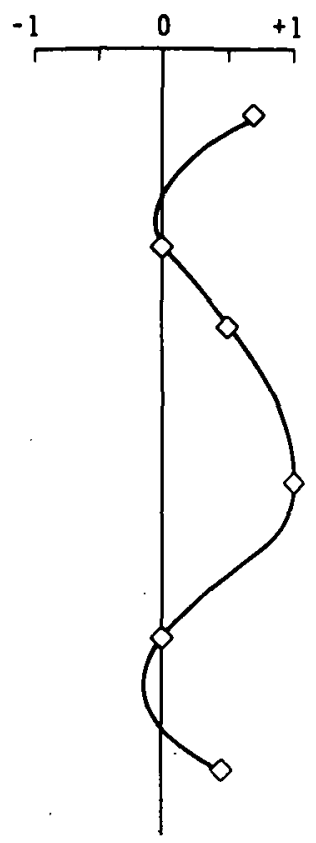

MODE 3

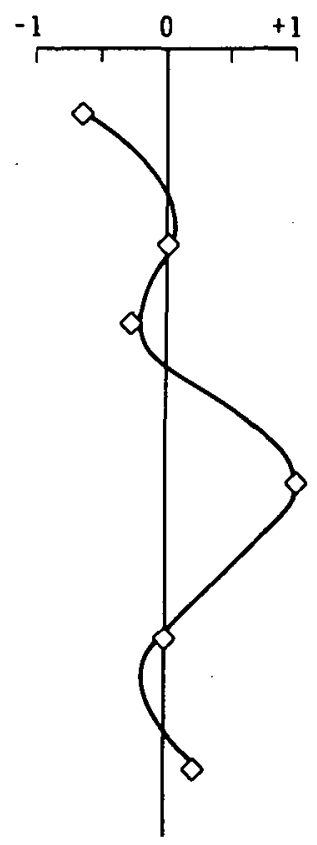

MODE 4

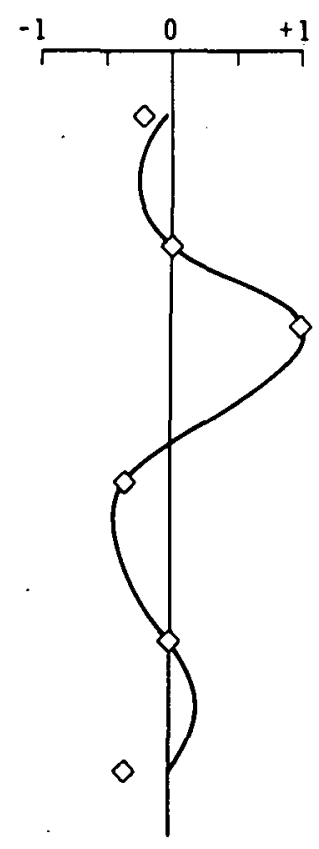

MODE 5

Figure 7. Orbiter Mode Shapes for Pin-Slip Configurations (b) M2 Mass Condition 
TABLE IV (a). COMPONENT NATURAL FREQUENCIES AND MODAL MASS FOR PIN-SLIP SUPPORT

(Booster)

\begin{tabular}{|c|c|c|c|c|c|c|}
\hline Configuration & \multicolumn{6}{|c|}{ Mode Number } \\
\hline B-P-Ll-Dl-Ml & 1 & 2 & 3 & 4 & 5 & 6 \\
\hline Theoretical frequency $(\mathrm{Hz})^{*}$ & 6.18 & 13.12 & 33.09 & 50.68 & 59.96 & 94.14 \\
\hline Experimental frequency (Hz) & 6.31 & 13.12 & 33.43 & 47.00 & 58.00 & 91.40 \\
\hline Percent difference & -2.06 & 0.00 & -1.02 & +7.83 & +3.38 & +3.00 \\
\hline
\end{tabular}

\begin{tabular}{|c|c|c|c|c|c|c|}
\hline B-P-L1-D2-M1 & 1 & 2 & 3 & 4 & 5 & 6 \\
\hline Theoretical frequency $^{*}(\mathrm{~Hz})$ & 6.18 & 13.12 & 33.09 & 50.68 & 59.96 & 94.14 \\
\hline Experimental frequency $(\mathrm{Hz})$ & 6.26 & 13.09 & 33.20 & 46.90 & 57.80 & 90.90 \\
\hline Percent difference & -1.28 & +0.23 & -0.33 & +8.06 & +3.74 & +3.56 \\
\hline
\end{tabular}

\begin{tabular}{|c|c|c|c|c|c|c|}
\hline B-P-Ll-D3-M1 & 1 & 2 & 3 & 4 & 5 & 6 \\
\hline Theoretical frequency $^{*}(\mathrm{~Hz})$ & 6.18 & 13.12 & 33.09 & 50.68 & 59.96 & 94.14 \\
\hline Experimental frequency $(\mathrm{Hz})$ & 6.20 & 13.06 & 33.15 & 46.80 & 57.90 & 91.00 \\
\hline Percent difference & -0.30 & +0.46 & -0.18 & +8.29 & +3.56 & +3.45 \\
\hline
\end{tabular}

\begin{tabular}{|c|c|c|c|c|c|c|}
\hline B-P-Ll-D2-M2 & 1 & 2 & 3 & 4 & 5 & 6 \\
\hline Theoretical frequency ${ }^{*}(\mathrm{~Hz})$ & 6.57 & 13.28 & 35.48 & 52.37 & 69.09 & 150.06 \\
\hline Experimental frequency $(\mathrm{Hz})$ & 6.64 & 13.75 & 35.62 & 48.20 & 66.20 & 122.90 \\
\hline Percent difference & -1.05 & -3.42 & -0.39 & +8.65 & +4.36 & +22.10 \\
\hline
\end{tabular}

\begin{tabular}{|c|c|c|c|c|c|c|}
\hline Normalized Modal Mass & 1 & 2 & 3 & 4 & 5 & 6 \\
\hline M1 mass configuration (Kgs) & 2.50 & 3.55 & 4.85 & 9.60 & 4.32 & 3.52 \\
\hline M2 mass configuration (Kgs) & 2.21 & 3.47 & 3.15 & 12.96 & 2.73 & 2.78 \\
\hline
\end{tabular}

*Undamped eigenvalue results 
TABLE IV (b). COMPONENT NATURAL FREQUENCIES AND MODAL MASS FOR PIN-SLIP SUPPORT

(Orbiter)

\begin{tabular}{|c|c|c|c|c|c|c|}
\hline Configuration & \multicolumn{6}{|c|}{ Mode Number } \\
\hline O-P-Ll-Dl-Ml & 1 & 2 & 3 & 4 & 5 & 6 \\
\hline Theoretical frequency ${ }^{*}(\mathrm{~Hz})$ & 10.52 & 18.58 & 37.22 & 57.34 & 93.86 & $\ldots$ \\
\hline Experimental frequency $(\mathrm{Hz})$ & 10.62 & 18.40 & 36.73 & 53.30 & 92.10 & $\ldots$ \\
\hline Percent difference & -0.94 & +0.98 & +1.33 & +7.58 & +1.91 & $\ldots$ \\
\hline
\end{tabular}

\begin{tabular}{|l|c|c|c|c|c|c|}
\hline O-P-L1-D2-M1 & 1 & 2 & 3 & 4 & 5 & 6 \\
\hline Theoretical frequency ${ }^{*}(\mathrm{~Hz})$ & 10.52 & 18.58 & 37.22 & 57.34 & 93.86 & $\ldots$ \\
\hline Experimental frequency (Hz) & 10.67 & 18.35 & 36.67 & 53.10 & 92.00 & $\ldots$ \\
\hline Percent difference & -1.40 & +1.25 & +1.50 & +7.98 & +2.02 & $\ldots$ \\
\hline
\end{tabular}

\begin{tabular}{|l|c|c|c|c|c|c|}
\hline \multicolumn{1}{|c|}{ O-P-L1-D3-M1 } & 1 & 2 & 3 & 4 & 5 & 6 \\
\hline Theoretical frequency ${ }^{*}(\mathrm{~Hz})$ & 10.52 & 18.58 & 37.22 & 57.34 & 93.86 & $\ldots$ \\
\hline Experimental frequency $(\mathrm{Hz})$ & 10.58 & 18.30 & 36.62 & 53.10 & 92.20 & $\ldots$ \\
\hline Percent difference & -0.57 & +1.53 & +1.64 & +7.98 & +1.80 & $\ldots$ \\
\hline
\end{tabular}

\begin{tabular}{|c|c|c|c|c|c|c|}
\hline O-P-L1-D2-M2 & 1 & 2 & 3 & 4 & 5 & 6 \\
\hline Theoretical frequency $^{*}(\mathrm{~Hz})$ & 10.71 & 18.61 & 39.28 & 57.07 & 114.05 & $\ldots$ \\
\hline Experimental frequency $(\mathrm{Hz})$ & 10.73 & 18.40 & 38.55 & 53.60 & 106.80 & $\ldots$ \\
\hline Percent difference & -0.19 & +1.14 & +1.89 & +6.47 & +6.69 & $\ldots$ \\
\hline
\end{tabular}

\begin{tabular}{|c|c|c|c|c|c|c|}
\hline Normalized Modal Mass & 1 & 2 & 3 & 4 & 5 & 6 \\
\hline M1 mass configuration (Kgs) & 5.57 & 3.17 & 5.99 & 8.90 & 3.38 & $\ldots$ \\
\hline M2 mass configuration (Kgs) & 5.29 & 3.06 & 3.96 & 6.71 & 2.61 & $\ldots$ \\
\hline
\end{tabular}

*Undamped eigenvalue results 


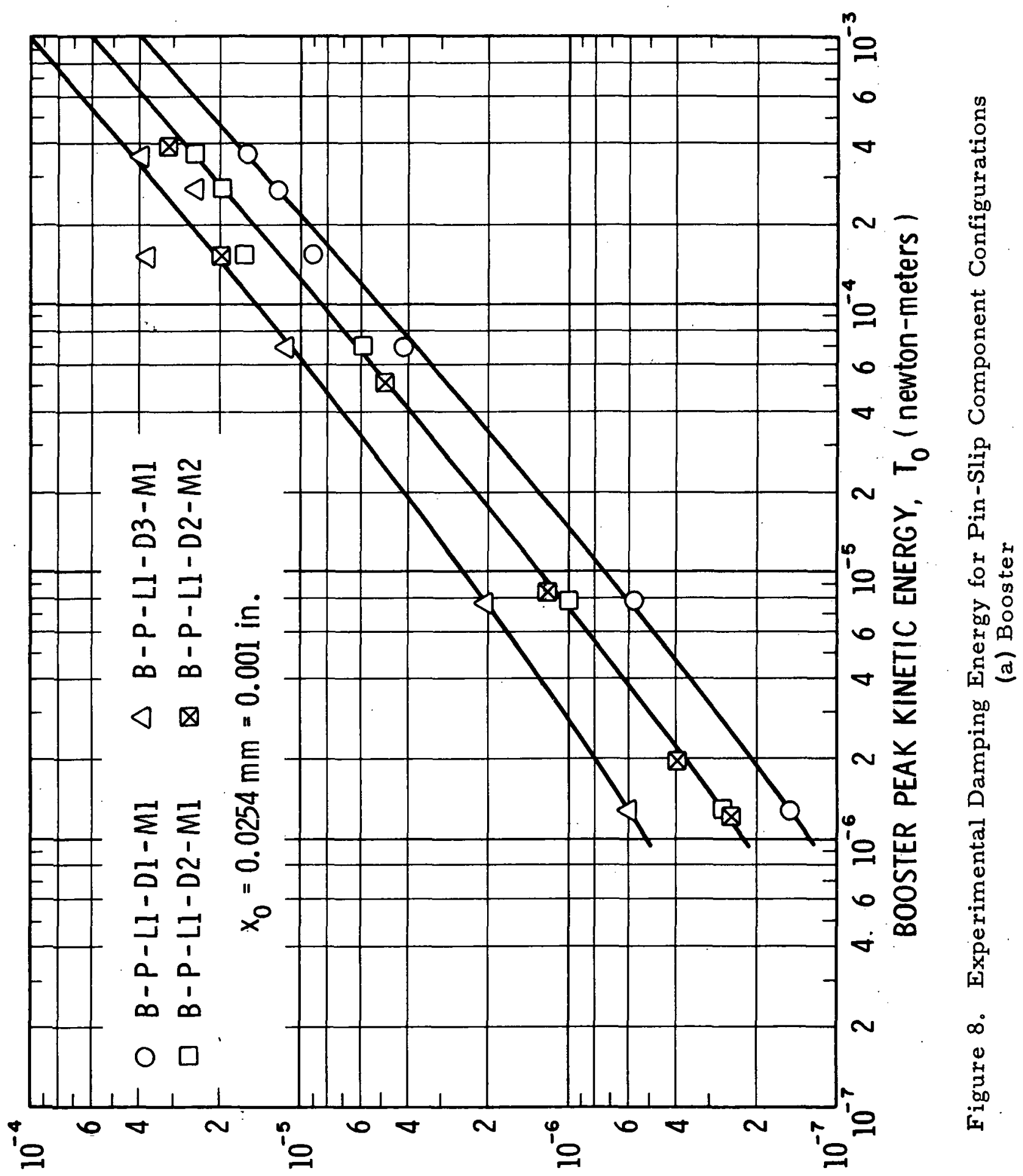

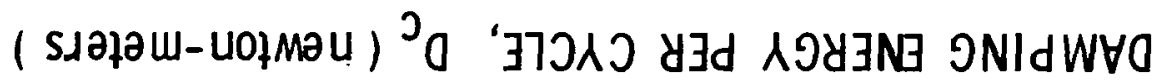




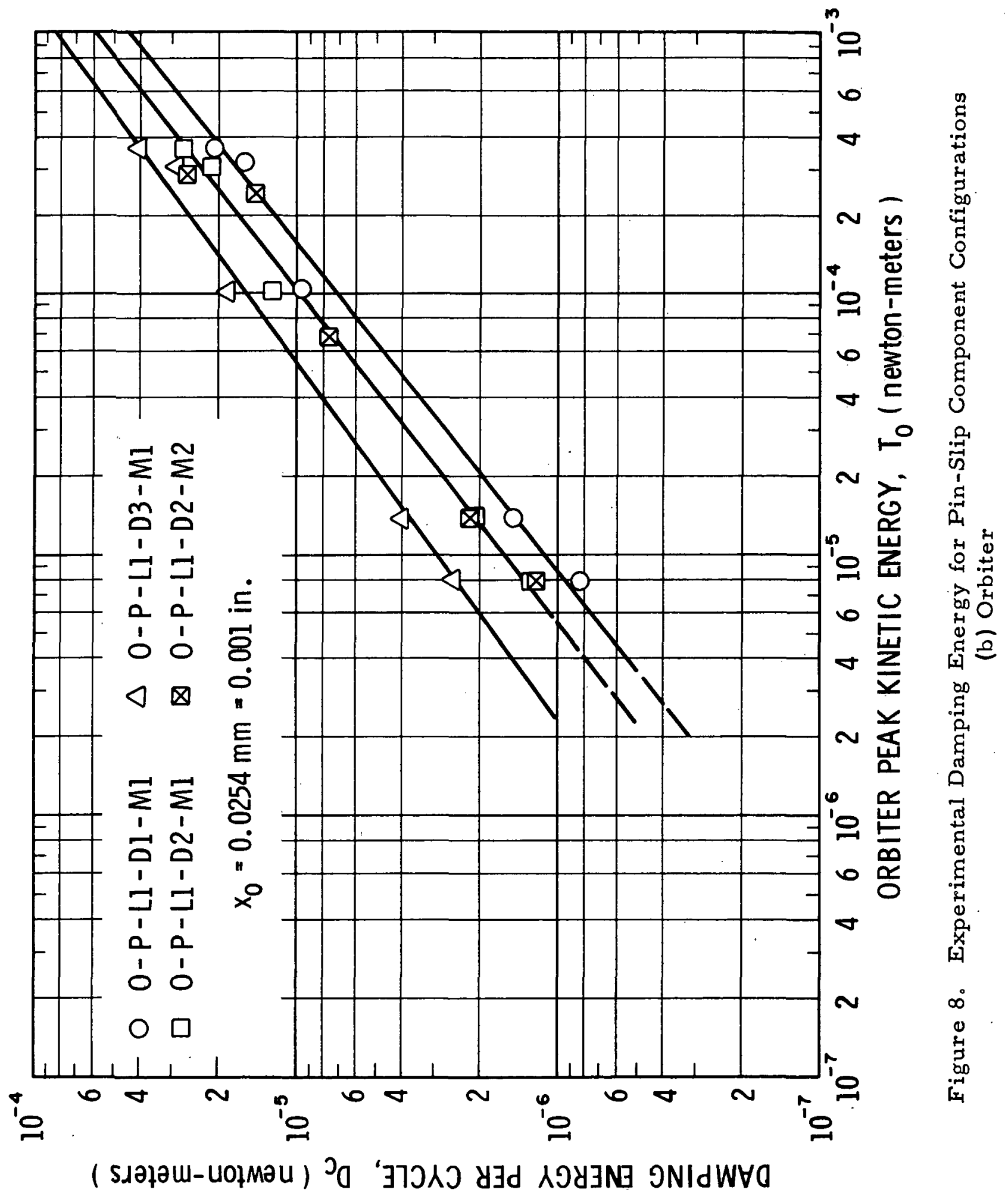


type damping mechanisms, the damping energy curves are congruent. Modal damping ratios in terms of critical damping are given in Table $V$ for both the Booster and Orbiter for various mass and damping conditions. Values for the DO (in air) conditions are also given to indicate that most of the damping was provided by the discrete oil dampers. The DO values for the Orbiter tend to be somewhat high, and are attributed to extraneous damping of accelerometer cables which was eliminated in subsequent tests.

3. Forced Response. To obtain forced responses, the Booster was excited with a constant force at Mass 10 in the lateral (z) direction and the Orbiter at Mass 4. Response measurements were also made at these locations in the lateral direction by means of an accelerometer attached to the component. Figures $9 \mathrm{a}$ and $9 \mathrm{~b}$ show the experimental and predicted forced responses for the Booster and Orbiter, respectively, in the form of response in $D B$ versus frequency. The responses are given in terms of velocity per unit force, with the response in the first mode taken as the reference. Therefore,

$$
D B=20 \log \frac{V / F}{V_{1} / F}
$$

where $V$ is the velocity at frequency $f$ and $V_{1}$ is the velocity in the first mode, while the force $F$ is held constant. These forced responses serve as an indication of the accuracy of-the analytical prediction procedure.

\section{B. Free-Free Configuration}

1. Natural Modes. The experimental and predicted normalized mode shapes for the Booster and Orbiter in the Ml mass configuration are shown in Figures 10 and 11 , respectively. Here again, as for the pin-slip configuration, these mode shapes are presented for the D2 damping condition. The experimental and theoretical frequencies and modal masses are presented in Table VI. Included are the three frequencies associated with the suspension system natural frequencies and denoted by $A=$ axial, $L=$ lateral, and $\mathrm{R}=$ rotational rigid-body motions, respectively. As noted from Table VI, agreement between experiment and theory is very good for the six Booster and five Orbiter modes. The percent difference between experimental and predicted natural frequencies is again based on the experimentally measured frequencies.

2. Damping. Experimental damping energy per cycle versus kinetic energy curves are shown in Figures $12 \mathrm{a}$ and $12 \mathrm{~b}$ for the Booster and Orbiter substructures, respectively. The curves were obtained by fairing through the experimental data points given by the symbols, and 
TABLE V. COMPONENT DAMPING FOR PIN-SLIP SUPPORT (Booster and Orbiter)

\begin{tabular}{|c|c|c|c|c|c|c|}
\hline Configuration & \multicolumn{6}{|c|}{ Mode Number } \\
\hline & 1 & 2 & 3 & 4 & 5 & 6 \\
\cline { 2 - 7 } B-P-Ll-D0-M1 & .0008 & .0010 & .0011 & .0012 & .0014 & .0016 \\
\hline O-P-Ll-D0-Ml & .0024 & .0016 & .0032 & .0024 & .0020 & $\ldots$ \\
\hline
\end{tabular}

\begin{tabular}{|l|l|l|l|l|l|l|}
\hline B-P-Ll-Dl-Ml & .0092 & .0057 & .0047 & .0034 & .0046 & .0032 \\
\hline O-P-Ll-Dl-M1 & .0079 & .0085 & .0074 & .0038 & .0045 & $\ldots$ \\
\hline
\end{tabular}

\begin{tabular}{|c|c|c|c|c|c|c|}
\hline B-P-L1-D2-M1 & .0164 & .0101 & .0066 & .0056 & .0087 & .0051 \\
\hline O-P-L1-D2-M1 & .0121 & .0117 & .0096 & .0053 & .0058 & $\ldots$ \\
\hline
\end{tabular}

\begin{tabular}{|c|c|c|c|c|c|c|}
\hline B-P-L1-D3-M1 & .0365 & .0200 & .0119 & .0071 & .0191 & .0080 \\
\hline O-P-L1-D3-M1 & .0244 & .0223 & .0142 & .0070 & .0087 & $\ldots$ \\
\hline
\end{tabular}

\begin{tabular}{|c|c|c|c|c|c|c|}
\hline B-P-LI-D0-M2 & .0013 & .0025 & .0019 & .0026 & .0022 & .0033 \\
\hline O-P-L1-D0-M2 & .0020 & .0015 & .0037 & .0028 & .0017 & $\ldots$ \\
\hline
\end{tabular}

\begin{tabular}{|c|c|c|c|c|c|c|}
\hline B-P-L1-D2-M2 & .0158 & .0112 & .0074 & .0063 & .0101 & .0116 \\
\hline O-P-L1-D2-M2 & .0121 & .0128 & .0086 & .0047 & .0075 & $\ldots$ \\
\hline
\end{tabular}




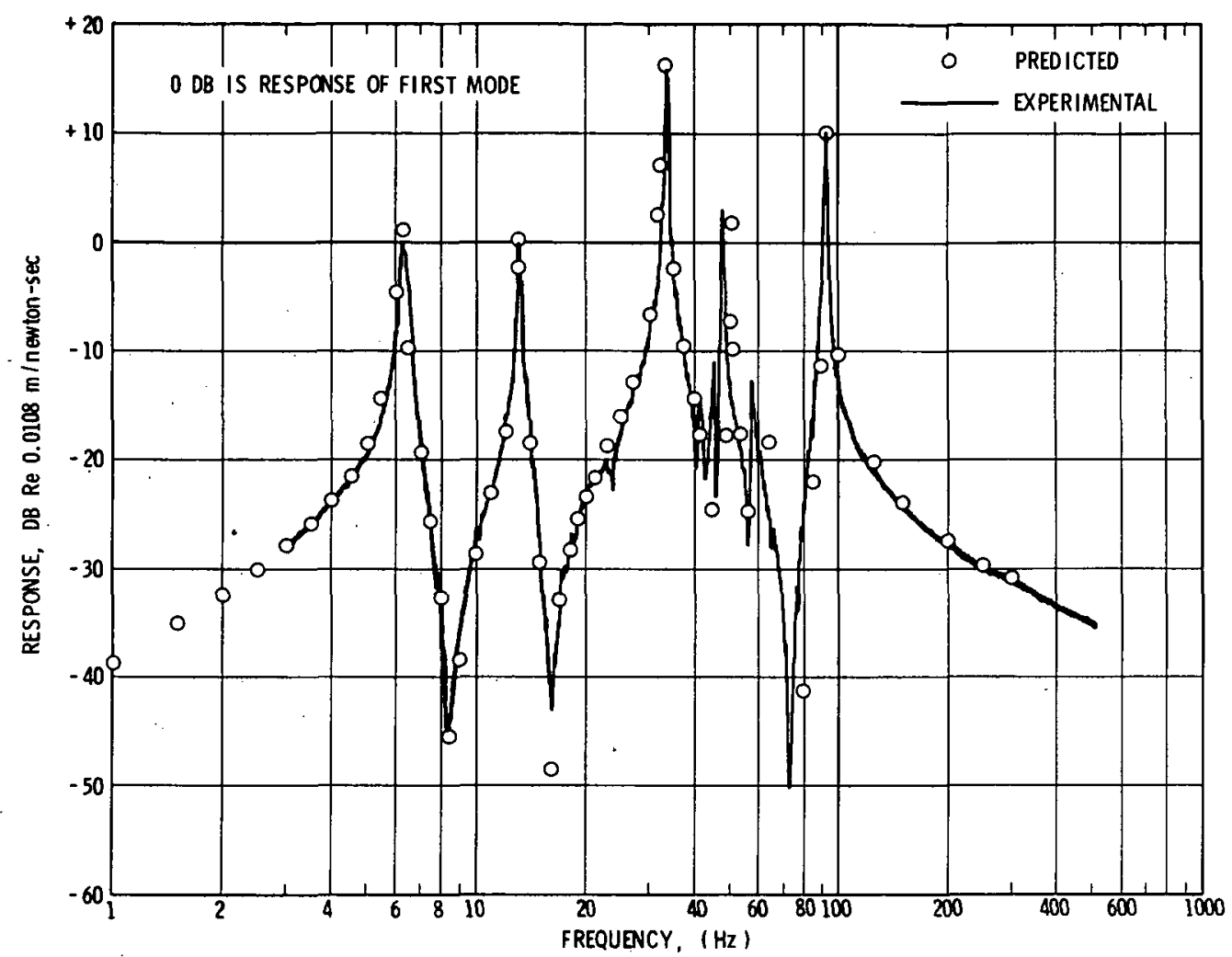

Figure 9a. Booster Component Response at Mass 10 for B-P-L1-D2-M1 Configuration

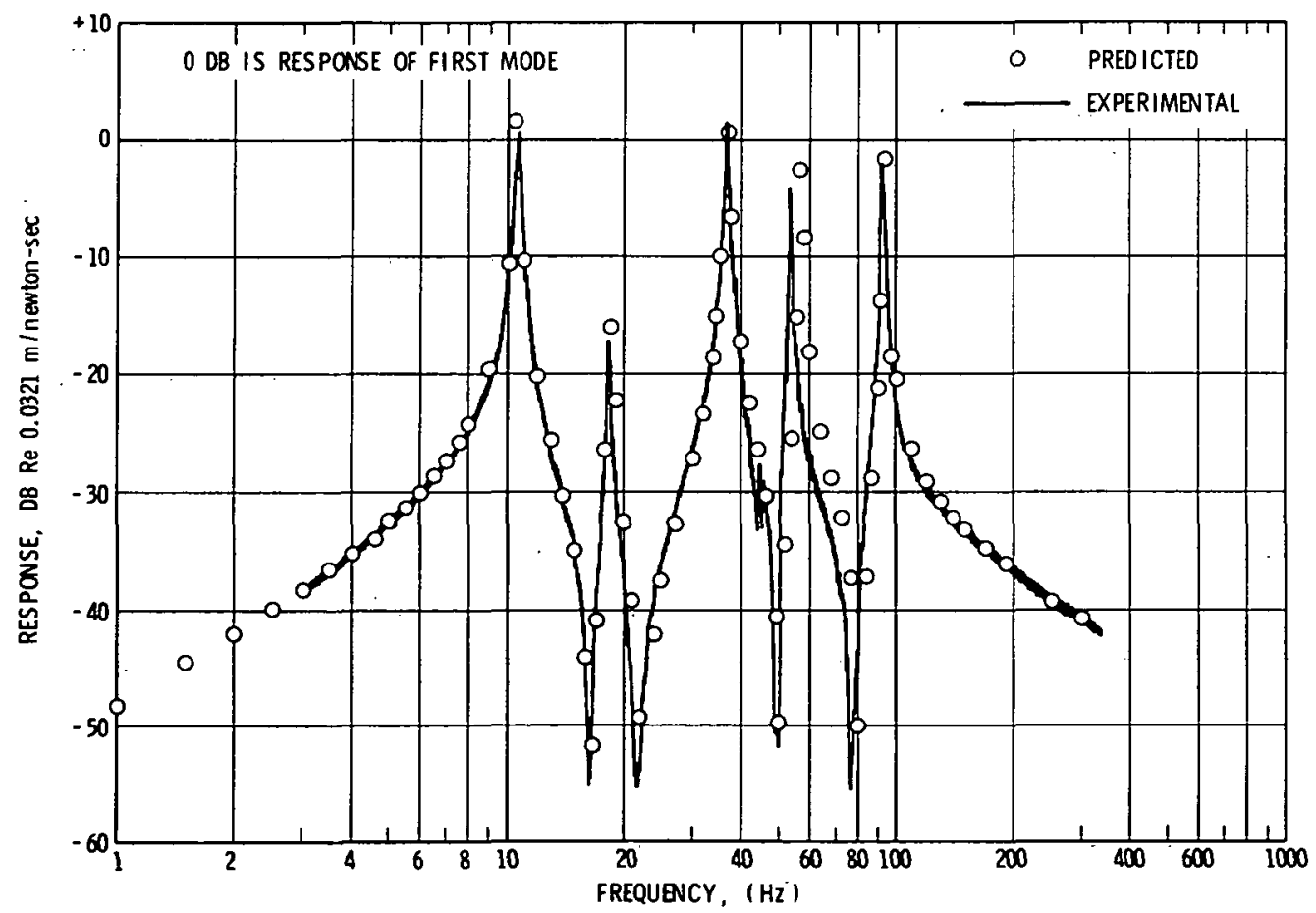

Figure 9b. Orbiter Component Response at Mass 4 for O-P-L1-D2-M1 Configuration 

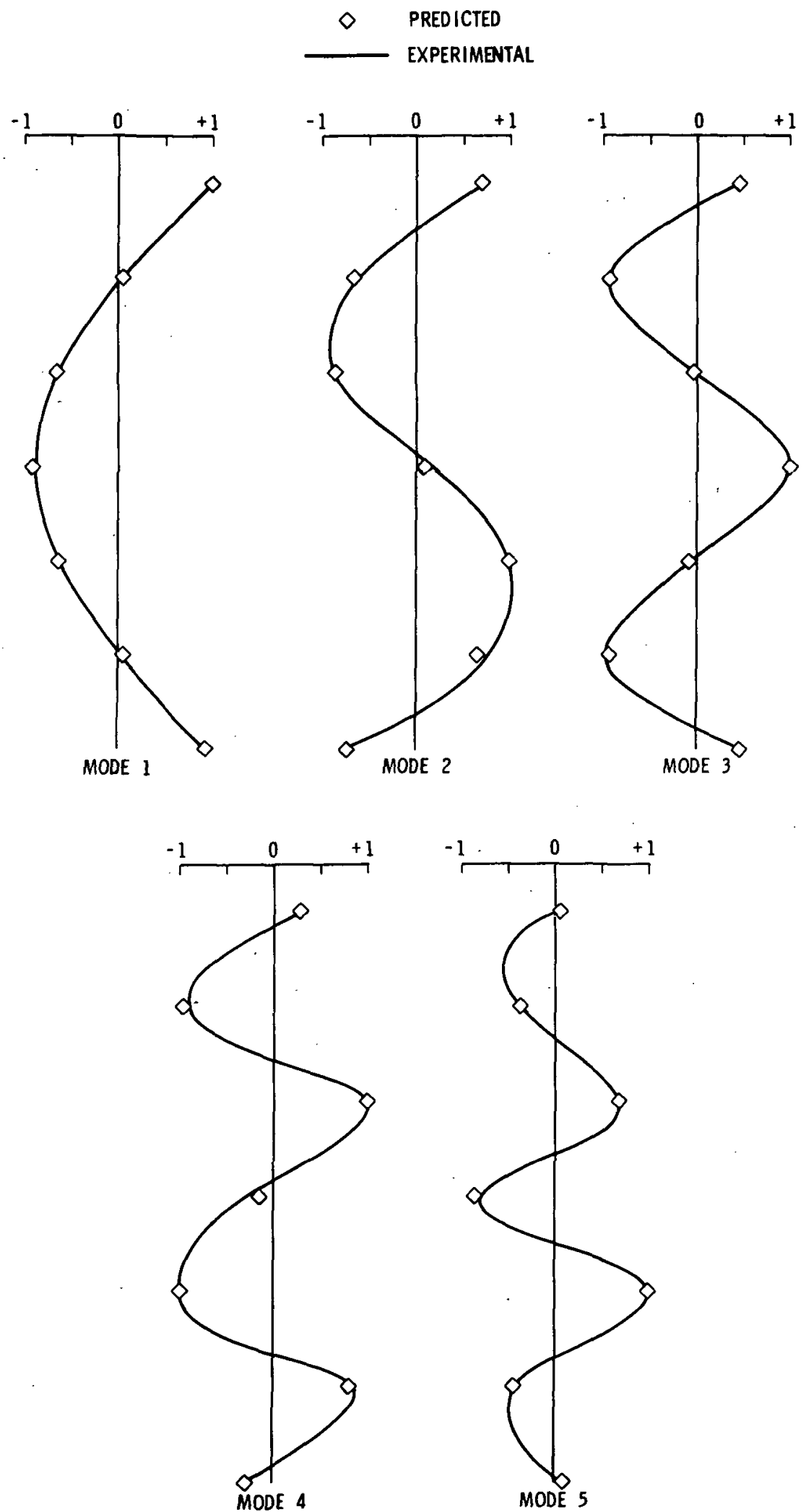

Figure 10. Booster Mode Shapes for Free-Free ConfigurationsMI Mass Condition 


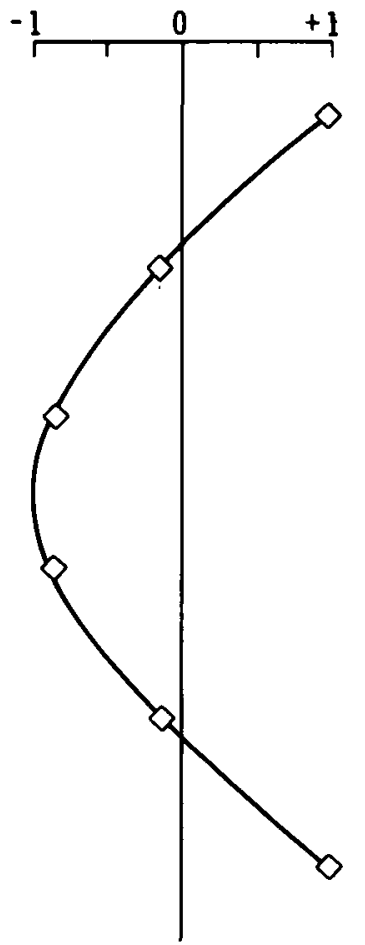

MODE 1

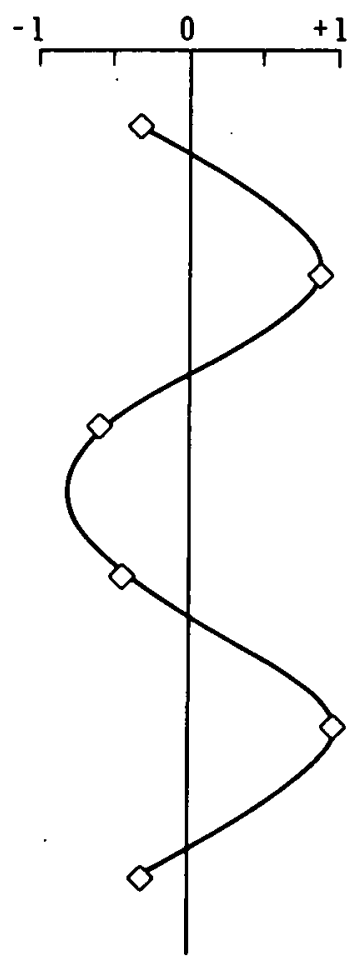

MODE 3

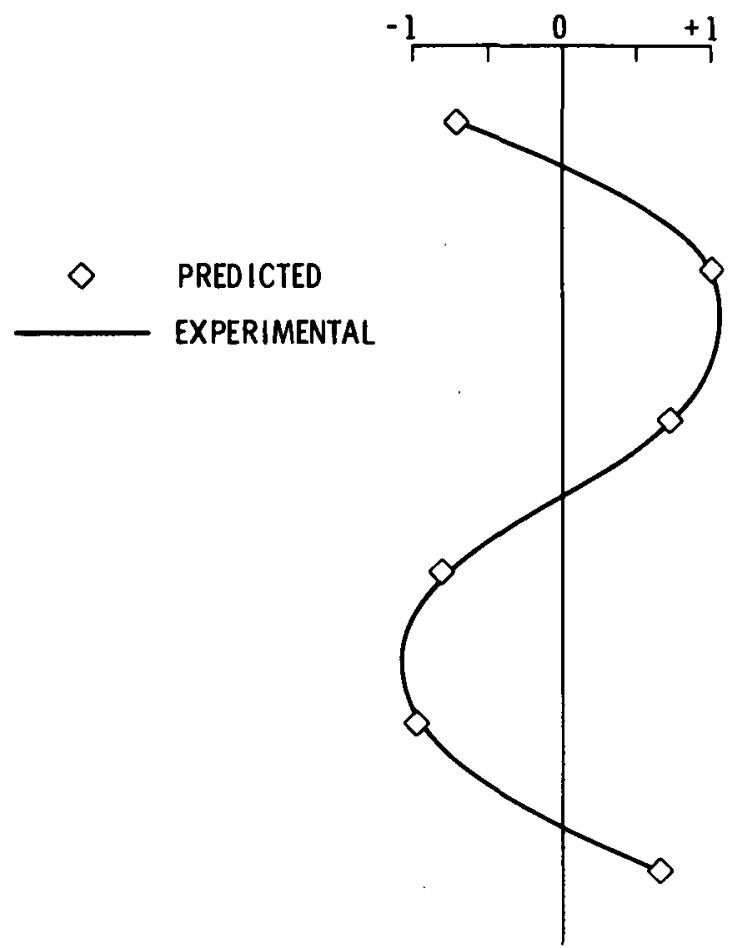

MODE 2

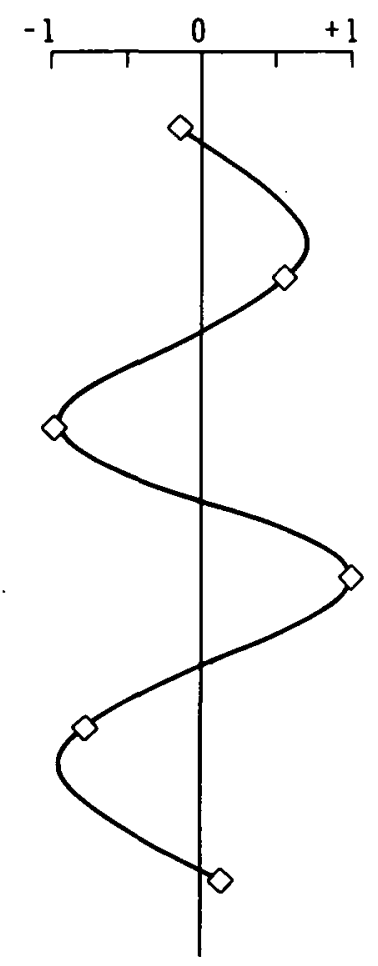

MODE 4

Figure 11. Orbiter Mode Shapes for Free-Free ConfigurationsMl Mass Condition 
TABLE VI. COMPONENT NATURAL FREQUENCIES AND MODAL MASS FOR FREE-FREE CONDITION

\section{(Booster and Orbiter)}

\begin{tabular}{|c|c|c|c|c|c|c|c|c|c|}
\hline Configuration & \multicolumn{9}{|c|}{ Mode Number } \\
\hline B-F-LO-D2-M1 & A & $L$ & $R$ & 1 & 2 & 3 & 4 & 5 & 6 \\
\hline Theoretical frequency $^{*}(\mathrm{~Hz})$ &.-- &.- & -- & 8.53 & 24.08 & 45.72 & 75.25 & 106.10 & 203.40 \\
\hline Experimental frequency (Hz) & 0.60 & 3.15 & 3.16 & 8.66 & 24.41 & 46.20 & 76.20 & 109.20 & 196.10 \\
\hline Percent difference &.-- & $-\ldots$ & $-\ldots$ & -1.50 & -1.35 & -1.04 & -1.25 & -2.84 & +3.72 \\
\hline
\end{tabular}

\begin{tabular}{|l|c|c|c|c|c|c|c|c|c|}
\hline O-F-LO-D2-Ml & $A$ & $L$ & $R$ & 1 & 2 & 3 & 4 & 5 & 6 \\
\hline Theoretical frequency $(\mathrm{Hz})$ & $\ldots--$ & $\ldots$ & $\ldots$ & 11.61 & 33.11 & 64.56 & 98.81 & 203.90 & $\ldots$ \\
\hline Experimental frequency $(\mathrm{Hz})$ & 0.77 & 3.57 & 3.75 & 11.72 & 33.19 & 64.60 & 100.70 & 200.10 & $\ldots$ \\
\hline Percent difference & $\ldots--$ & $\ldots$ &.-- & -0.94 & -0.24 & -0.06 & -1.88 & +1.90 & $\ldots$ \\
\hline
\end{tabular}

\begin{tabular}{|l|c|c|c|c|c|c|c|c|c|}
\hline \multicolumn{1}{|c|}{ B-F-L0-D3-Ml } & A & L & R & 1 & 2 & 3 & 4 & 5 & 6 \\
\hline Theoretical frequency $^{*}(\mathrm{~Hz})$ &.-- &.-- & -- & 8.53 & 24.08 & 45.72 & 75.25 & 106.10 & 203.40 \\
\hline Experimental frequency (Hz) & 0.60 & 3.15 & 3.16 & 8.62 & 24.50 & 46.10 & 76.30 & 109.10 & 196.20 \\
\hline Percent difference & --- & --- &.-- & -1.04 & -1.71 & -0.82 & -1.38 & -2.75 & +3.67 \\
\hline
\end{tabular}

\begin{tabular}{|l|c|c|c|c|c|c|c|c|c|}
\hline O-F-LO-D3-M1 & $A$ & $L$ & $R$ & 1 & 2 & 3 & 4 & 5 & 6 \\
\hline Theoretical frequency $(\mathrm{Hz})$ & $\ldots$ & $\ldots$ & $\ldots$ & 11.61 & 33.11 & 64.56 & 98.81 & 203.90 & $\ldots$ \\
\hline Experimental frequency $(\mathrm{Hz})$ & 0.72 & 3.58 & 3.23 & 11.68 & 33.20 & 64.60 & 101.00 & 201.50 & $\ldots$ \\
\hline Percent difference & $\ldots$ & $\ldots$ & $\ldots$ & -0.60 & -0.27 & -0.06 & -2.17 & +1.19 & $\ldots$ \\
\hline
\end{tabular}

\begin{tabular}{|c|c|c|c|c|c|c|c|c|c|}
\hline Normalized Modal Mass & A & L & $\mathrm{R}^{\dagger}$ & 1 & 2 & 3 & 4 & 5 & 6 \\
\hline $\begin{array}{c}\text { Booster } \\
\text { Ml mass configuration (Kgs) }\end{array}$ & 12.26 & 12.26 & 4.74 & 6.46 & 6.65 & 6.30 & 7.22 & 4.69 & 9.18 \\
\hline $\begin{array}{c}\text { Orbiter } \\
\text { Ml mass configuration (Kgs) }\end{array}$ & 10.44 & 10.44 & 5.72 & 6.62 & 7.37 & 4.69 & 5.71 & 10.24 & $\ldots$ \\
\hline
\end{tabular}

$$
\begin{aligned}
& A=\text { Axial rigid body } \quad L=\text { Lateral rigid body } \quad R=\text { Rotational rigid body } \\
& { }^{*} \text { Undamped eigenvalue results } \\
& \left.+I / \ell^{2} \text { ( } \ell=\text { distance from } \mathrm{cg} \text { to bottom of component }\right)
\end{aligned}
$$




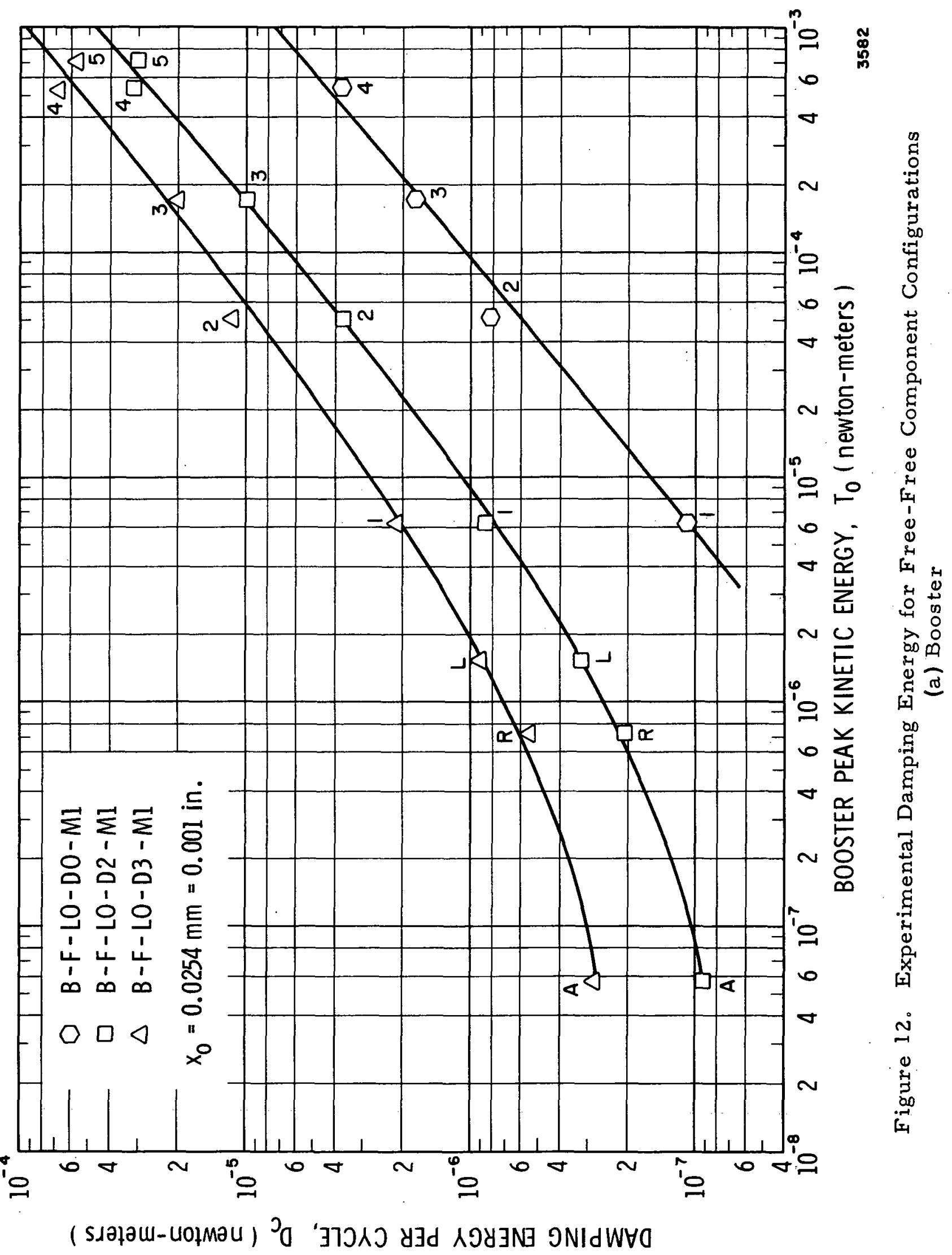




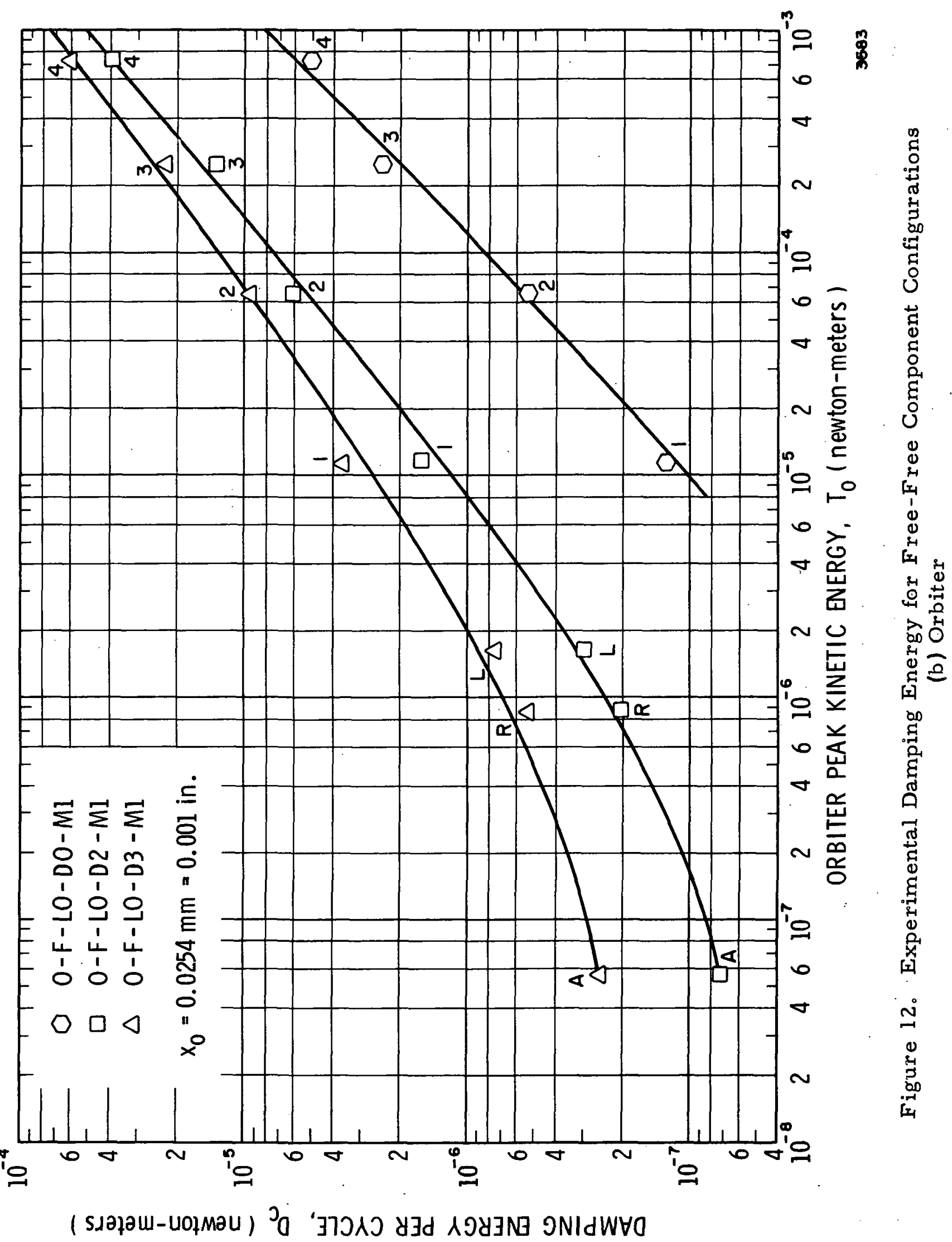


results are shown for the $\mathrm{D} 0, \mathrm{D} 2$, and $\mathrm{D} 3$ damping conditions in the $\mathrm{Ml}$ mass configuration. In additon to the damping values associated with the natural modes, damping values are plotted for the rigid-body motions associated with the axial, lateral, and rotational suspension system natural frequencies. Recall that due to the nature of the damping mechanism employed in the experiments, damping in rigid-body modes is also present at the non-resonant frequencies of the component. It was then assumed for the purpose of analysis that the damping associated with rigid-body motion corresponded to the damping obtained at the suspension system natural frequencies. Experimentally determined elastic mode damping ratios as well as rigid-body damping ratios are given in Table VII for the Booster and Orbiter substructures. :

3. Forced Response. To obtain forced responses, the Booster was excited with a constant force at Mass 10 in the lateral (z) direction and the Orbiter at Mass 4. Response measurements were also made at these locations in the lateral direction by means of an accelerometer attached to the component. Figures $13 \mathrm{a}$ and $13 \mathrm{~b}$ show the experimental and predicted forced responses for the Booster and Orbiter, respectively, in the form of response in DB versus frequency. As for the pin-slip configuration, the responses are given in terms of velocity per unit force, with the response in the first elastic mode taken as the reference value. As with the pin-slip configurations, these responses serve only as an indication of the accuracy of the analytical model, and offer nothing new in dynamic analysis. New findings will be presented for the System results. 


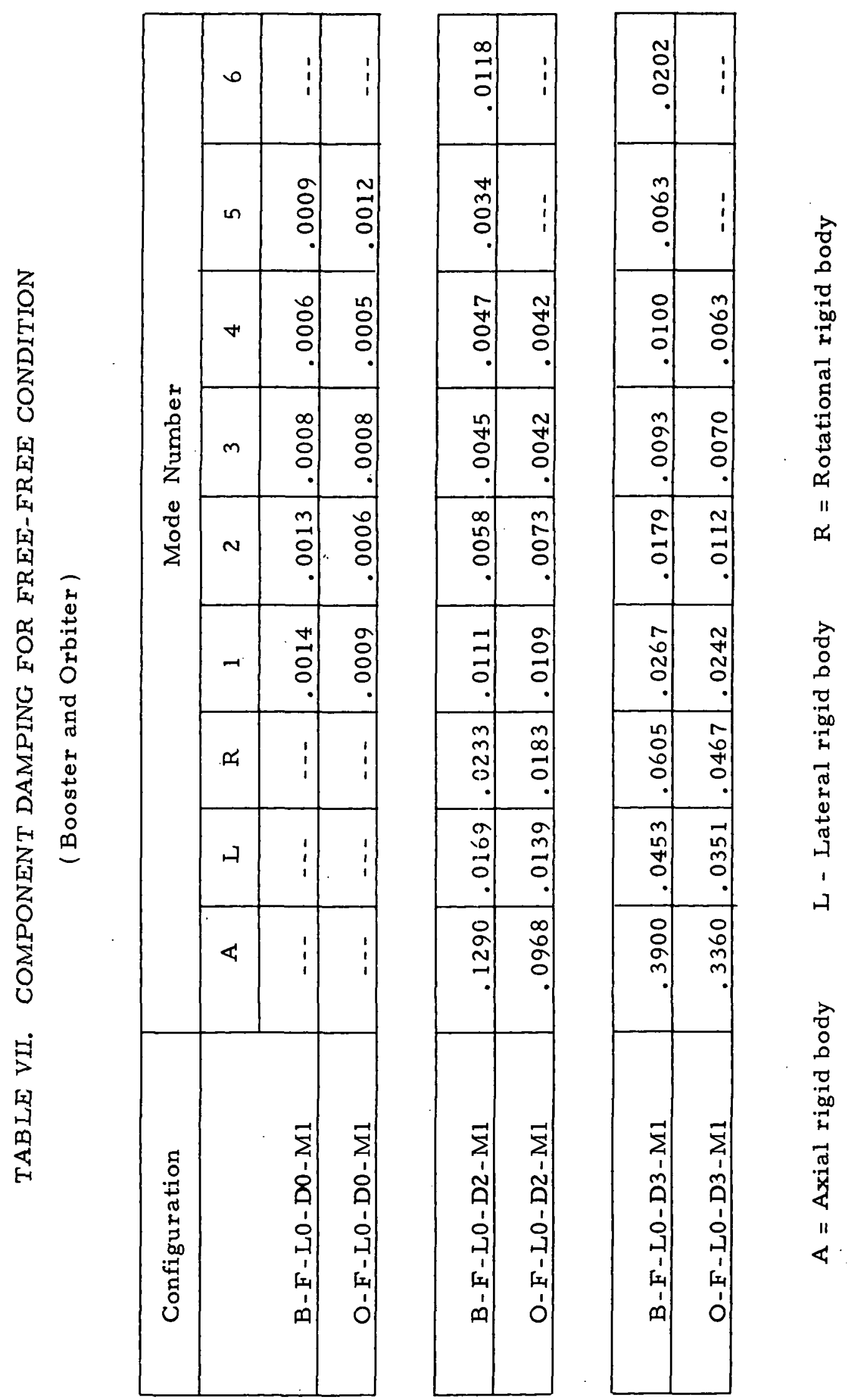




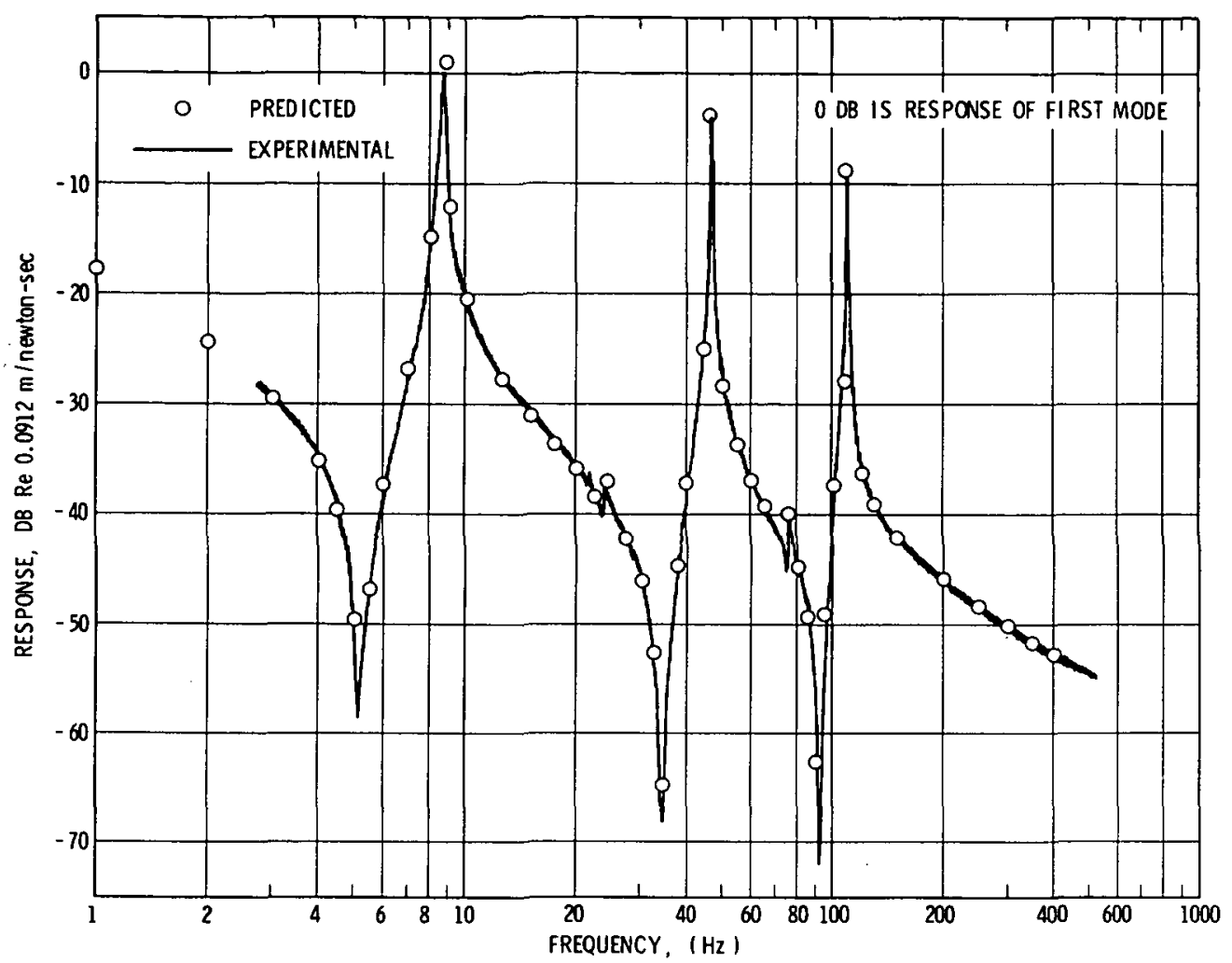

Figure 13a. Booster Component Response at Mass 10 for B-F-L0-D2-M1 Configuration

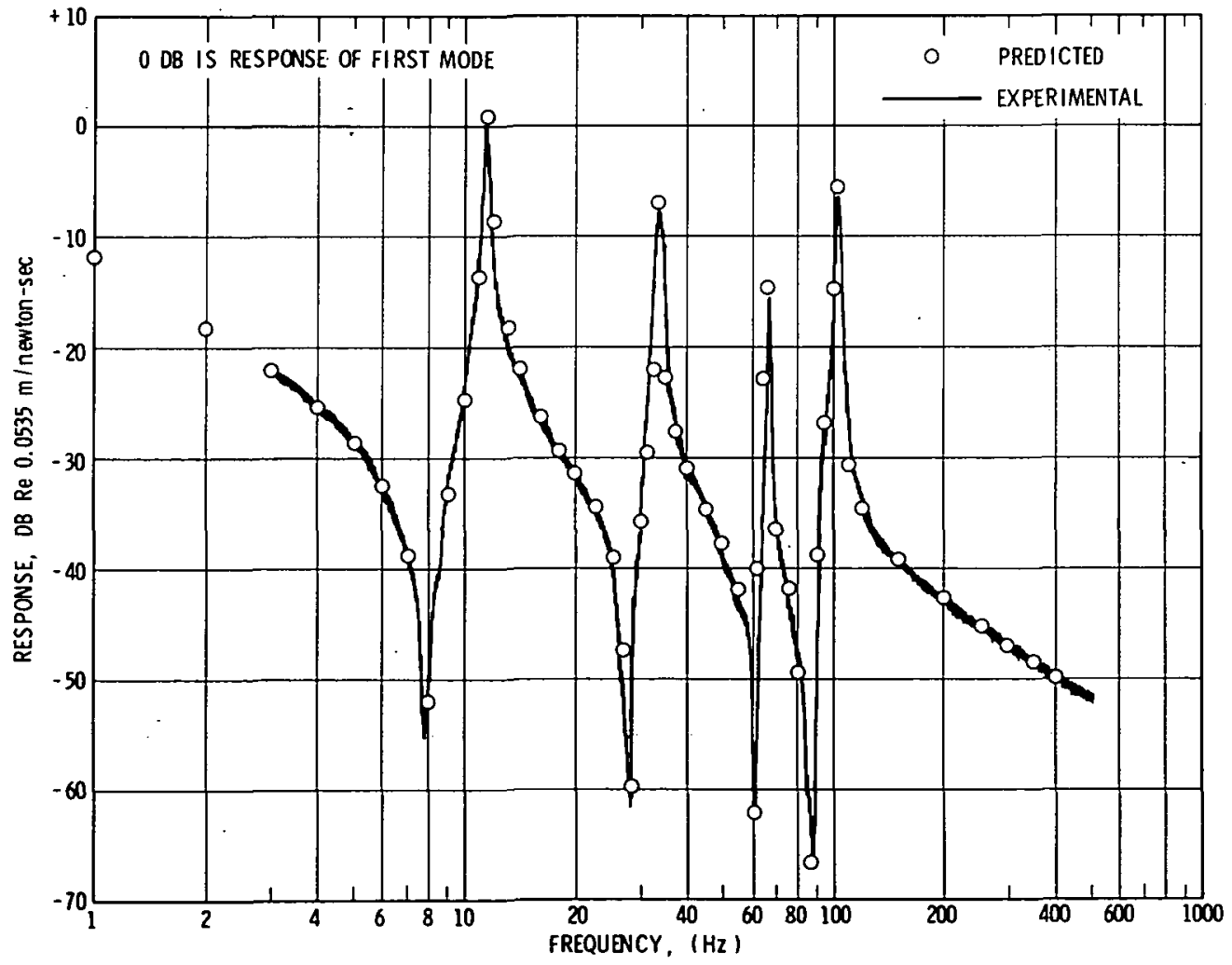

Figure 13b. Orbiter Component Response at Mass 4 for O-F-L0-D2-M1 Configuration 


\section{SYNTHESIS OF SYSTEM DAMPING}

The comments in Section IV. A provide the basis for our method of damping synthesis. In particular, we hypothesize that the curves in Figures $8 \mathrm{a}, \mathrm{b}$ and $12 \mathrm{a}, \mathrm{b}$ provide a means of selecting an appropriate value of damping energy for each component, once we establish its peak kinetic energy of vibration at a given frequency and amplitude, regardless of the spatial shape it may sustain. In view of this hypothesis, we are now in position to predict the damping values for the combined System. We first perform an ordinary modal analysis of the undamped System. This provides the natural frequencies, mode shapes, and kinetic energies for a given modal amplitude $x_{0}$. Let $T_{0 s}, T_{0 B}$, and $T_{00}$ be the kinetic energies for the System, Booster, and Orbiter, respectively, in a given System mode. We note that either the Booster or Orbiter will have an amplitude $\dot{x}_{0}$ while the other component will have some smaller maximum amplitude in that mode. Since the energy curves (Figures $8 \mathrm{a}, \mathrm{b}$ and $12 \mathrm{a}, \mathrm{b})$ are plotted with constant amplitude $\mathrm{x}_{0}$, the peak displacement of the component having the smaller displacement is scaled so that its peak displacement is now $x_{0}$. (This is equivalent to selecting the damping energy from the curve of lower constant amplitude, were such a curve available.) The scale factor is obtained by dividing $x_{0}$ by $x^{\prime}$ where $x^{\prime} \leq x_{0}$, and is the maximum amplitude in a given mode on the component having the smaller amplitude. The scale factor may then be written

$$
S_{p}=x_{0} / x^{\prime} \geq 1
$$

For the component having amplitude $x_{0}$ we enter the appropriate energy curve and read the damping energy, $D_{0}$, corresponding to the kinetic energy $\mathrm{T}_{0}=\frac{1}{2} \mathrm{M} w^{2} \mathrm{x}_{0}^{2}$. For the component having an amplitude $\mathrm{x}^{\prime}$ we first multiply the kinetic energy by the scale factor squared so that $T_{0}^{\prime}=\frac{1}{2} M \omega^{2} x^{\prime 2} S_{f}^{2}$. We then enter the appropriate energy curve and read a damping energy, $D_{c}^{\prime}$. This damping energy, however, is based on an amplitude $\mathbf{x}_{0}$. To obtain the actual damping energy we simply divide $D_{c}^{\prime}$ by the scale factor squared to obtain actual damping energy, $D_{c}$. Once the $D_{c}$ values are determined for each component the System damping can then be predicted from

$$
\delta_{B}=\frac{D_{C B}+D_{c O}}{4 \pi T_{O S}}=\frac{D_{c S}}{4 \pi T_{O S}}
$$

The damping ratios obtained using this method are presented in Section VII. B for several system configurations. It should also be pointed out that the above scaling procedure is necessary, since Figures 8 and 12 indicate that damping energy is a nonlinear function of peak kinetic energy. 
Allowance for joint damping is accomplished by adding the damping energy of the joint which is calculated from the calibrated properties of the joint damper (see Section IV. C) and the relative velocity across the joint as given by the theoretical mode shape. The damping ratio for both the link and structure is then obtained by

$$
\zeta_{B, l}=\frac{D_{c s}+D_{c l}}{4 \pi T_{o s}}=\zeta_{B}+\zeta_{l}
$$




\section{SYSTEM RESULTS}

\section{A. Natural Modes}

System natural frequencies and mode shapes were obtained from synthesis of both the component pin-slip and component free-free modes. For the $M 1$ and $M 2$ mass configurations the first six pin-slip Booster and first five pin-slip Orbiter modes were used in the synthesis. The first five Booster free-free modes and the first four Orbiter free-free modes were used in the synthesis for the $M 1$ mass configuration only. Inspection of Ml mass configuration System modes and frequencies showed that the pin-slip component mode synthesis produced much better results at higher frequencies than did the free-free component mode synthesis. Therefore, only the pin-slip component modes were used in the M2 mass configuration System synthesis.

The System mode shapes obtained from pin-slip component modes and free-free component modes are shown in Figure 14 a for the $M 1$ mass configuration. It can be seen that the agreement between the experimental and predicted values is very good to excellent for the pin-slip case up to the tenth mode. It should be noted that the seventh and eighth System modes could not be determined experimentally, because of the location of the exciter. However, since the pin-slip component modes produced good results up to the tenth mode it was surmised that modes 7 and 8 would conform to the pin-slip analysis and are so indicated. Figure 14b shows the mode shapes obtained for the M2 mass configuration, and they also agree quite well with the experimental results.

Table VIII(a) shows the System natural frequencies and modal masses obtained from synthesis of the pin-slip component modes for the M1 and M2 mass configurations. It should be noted that three eigenvalue results are presented for the $M l$ mass configuration. These results correspond to 1) an analysis assuming zero damping, 2) an analysis using the D2 component damping results, and 3) an analysis using the D3 component damping results. These results were obtained from a complex eigenvalue analysis in which we tried to determine if System damping could be obtained by synthesizing the component damping matrices as outlined in Section III. D. 4. The differences in predicted frequencies for the undamped and the damped cases are, of course, very small and are presented here only for comparison. To assess the convergence properties inherent in assuming a finite number of component modes for synthesis, two other cases are presented in Table VIII(a), in which we synthesized three Booster and three Orbiter modes and then 

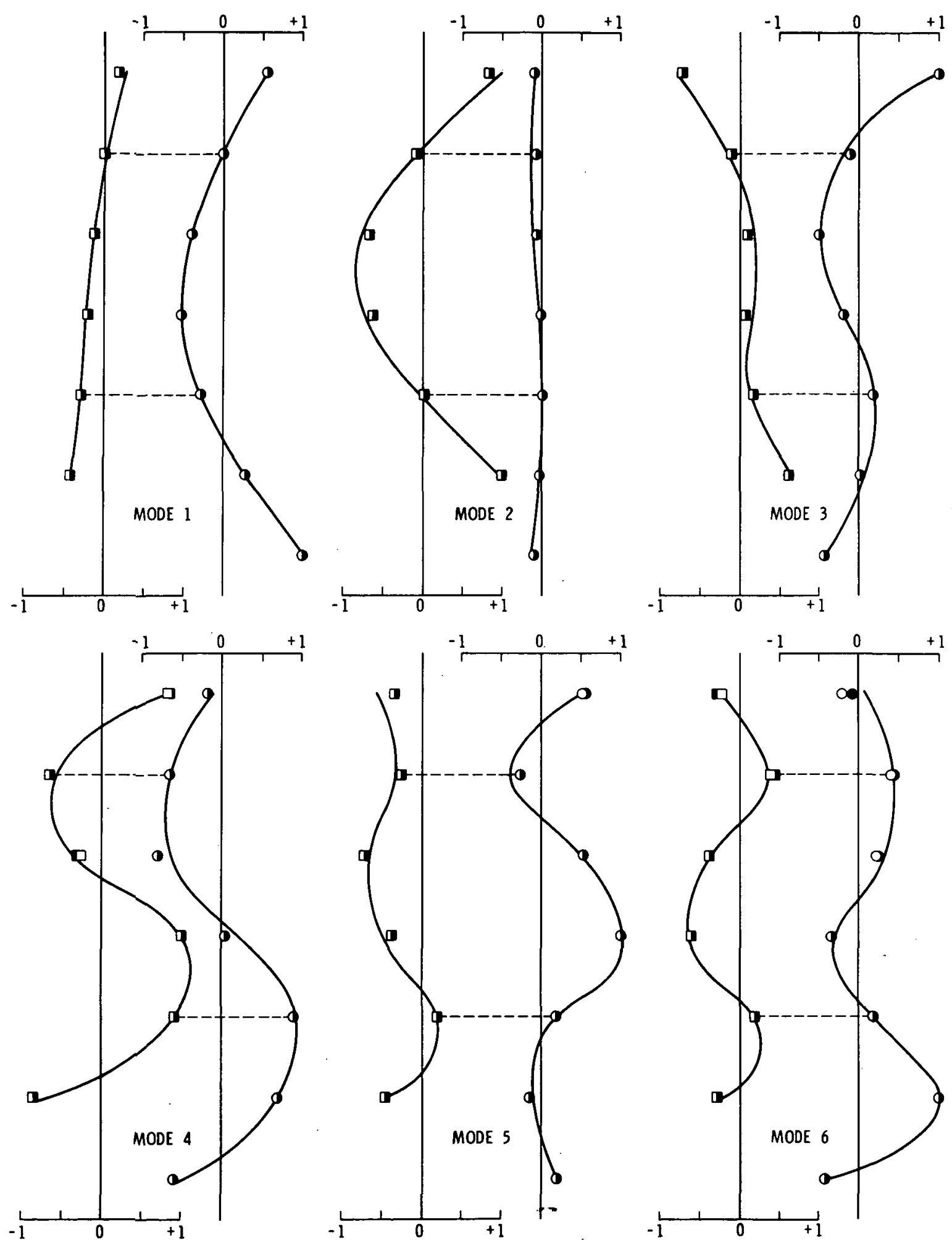

Figure 14. Combined System Mode Shapes

(a) M1 Mass Condition 
口 O PREDICTED USING FREE-FREE COMPONENT MODES

- - PREDICTED USING PIN-SLIP COMPONENT MODES EXPERIMENTAL
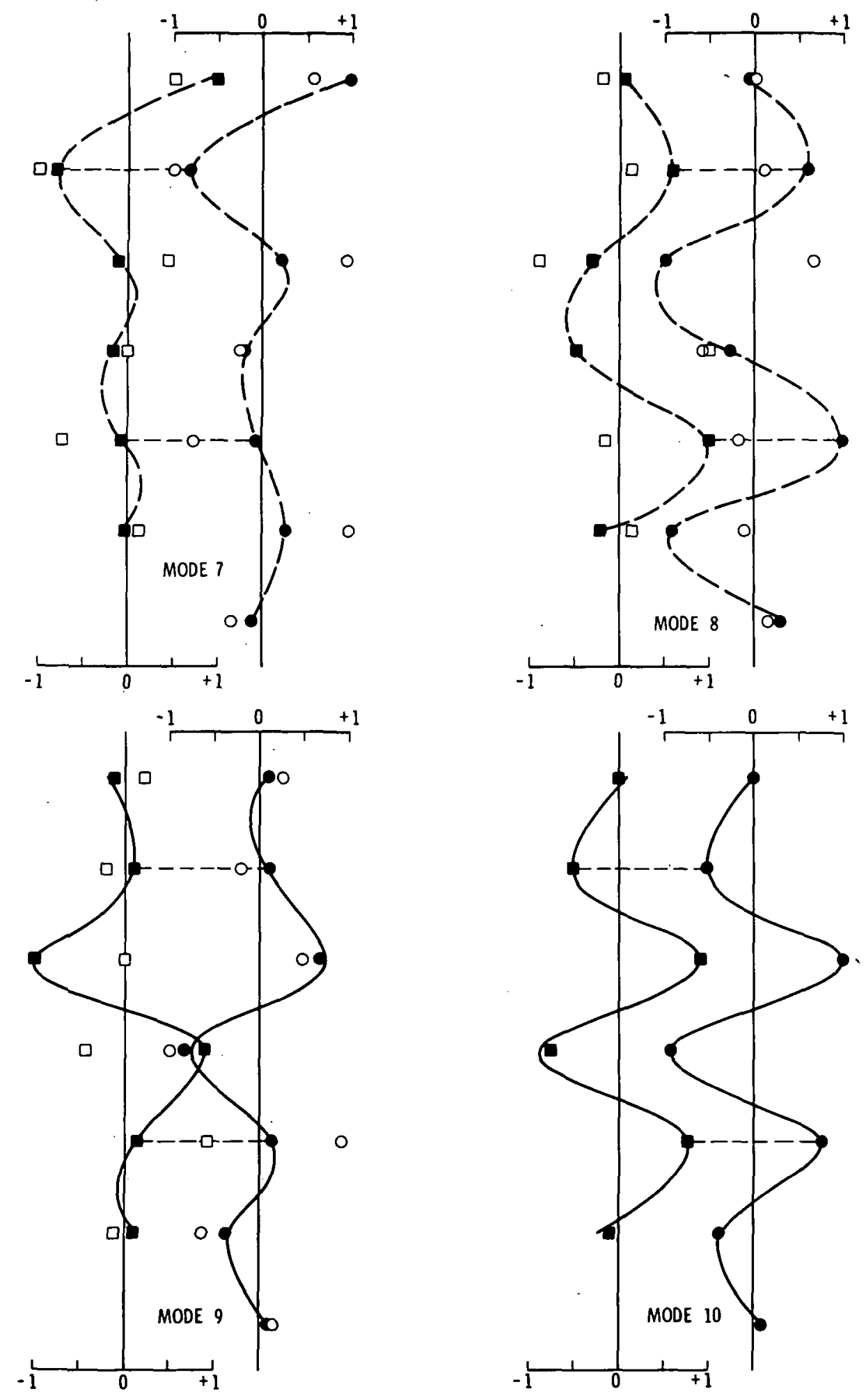

Figure 14. (Cont'd.) Combined System Mode Shapes (a) MI Mass Condition 

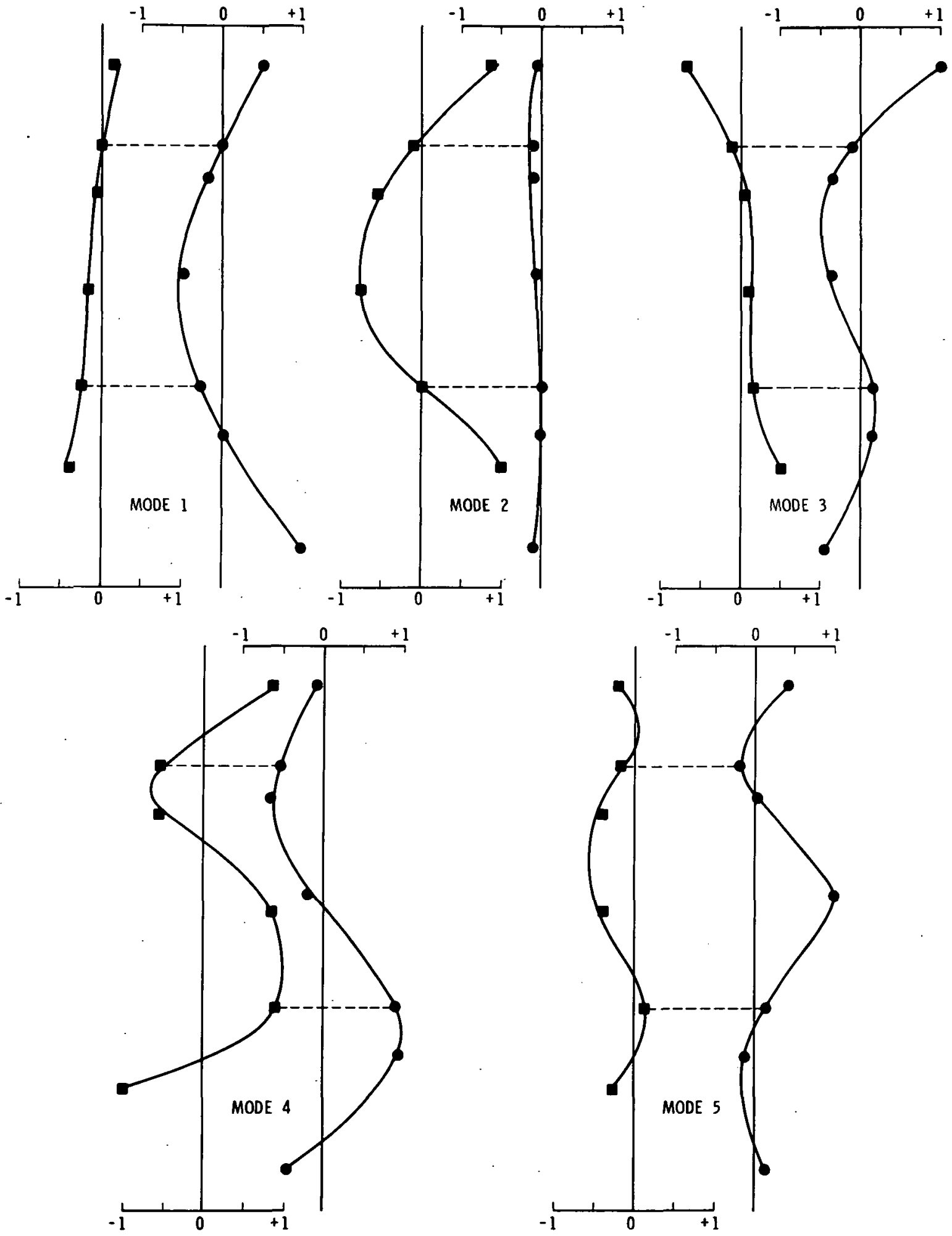

Figure 14. (Cont'd.) Combined System Mode Shapes (b) M2 Mass Condition 
- PREDICTEd USING PIN-SLIP COMPONENT MODES EXPERIMENTAL
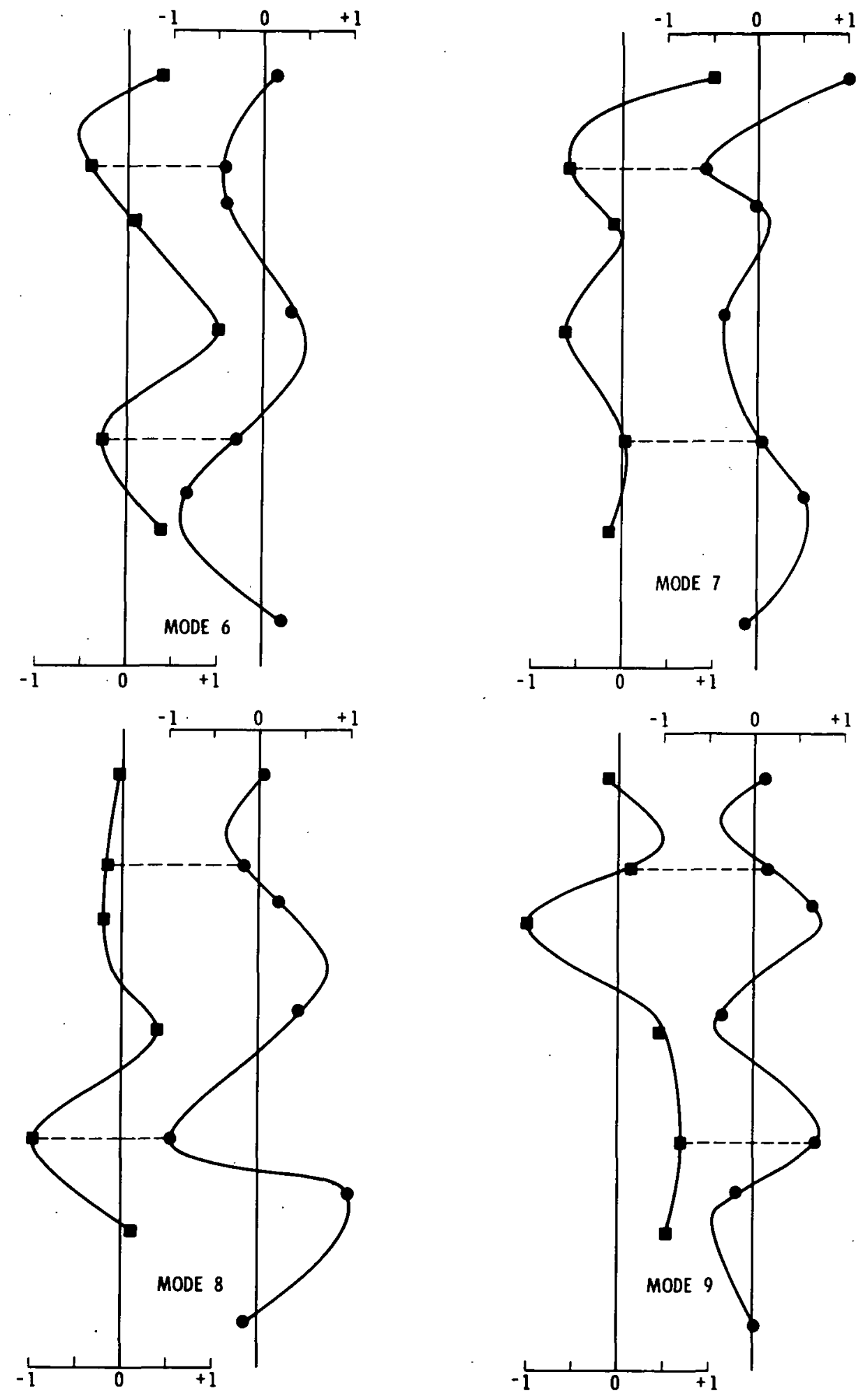

Figure 14. (Cont'd.) Combined System Mode Shapes (b) M2 Mass Condition 
TABLE VIII(a)

SYSTEM NATURAL FREQUENCIES AND MODAL MASS FROM SYNTHESIS OF PIN-SLIP COMPONENT MODES

Ml Mass Configuration

\begin{tabular}{|c|c|c|c|c|c|c|c|c|c|c|}
\hline \multirow{2}{*}{$\begin{array}{l}\text { Booster Modes } 1 \text { through } 6 \text { used. } \\
\text { Orbiter Modes } 1 \text { through } 5 \text { used. }\end{array}$} & \multicolumn{10}{|c|}{ Mode Number } \\
\hline & 1 & 2 & 3 & 4 & 5 & 6 & 7 & 8 & 9 & 10 \\
\hline Theoretical frequency ${ }^{1}(\mathrm{~Hz})$ & 7.31 & 11.02 & 16.25 & 27.38 & 35.74 & 49.63 & 55.87 & 74.92 & 85.67 & 108.81 \\
\hline Theoretical frequency ${ }^{2}(\mathrm{~Hz})$ & 7.30 & 11.01 & 16.25 & 27.37 & 35.73 & 49.63 & 55.86 & 74.91 & 85.67 & 108.79 \\
\hline Theoretical frequency ${ }^{3}(\mathrm{~Hz})$ & 7.29 & 11.01 & 16.24 & 27.33 & 35.69 & 49.62 & 55.83 & 74.91 & 85.65 & 108.72 \\
\hline Experimental frequency ( $\mathrm{Hz})$ & 7.35 & 11.13 & 16.34 & 26.79 & 34.56 & 49.73 & 56.00 & 76.00 & 87.07 & 108.64 \\
\hline
\end{tabular}

M1 Mass Configuration

Booster Modes 1 through 3 used.

Orbiter Modes 1 through 3 used.

Theoretical frequency $1 \mathrm{~Hz}$

Experimental frequency ( $\mathrm{Hz}$ )

Percent difference 4

\begin{tabular}{|c|c|c|c|c|c|c|c|c|c|}
\hline \multicolumn{10}{|c|}{ Mode Number } \\
\hline 1 & 2 & 3 & 4 & 5 & 6 & 7 & 8 & 9 & 10 \\
\hline 7.31 & 11.02 & 16.37 & 27.78 & 36.06 & 55.05 & $\ldots$ & $\therefore$ & $\cdots$ & $\ldots$ \\
\hline 7.35 & 11.13 & 16.34 & 26.79 & 34.56 & 49.73 & $\ldots$ & $=$ & $\ldots$ & $\ldots$ \\
\hline-0.54 & -0.99 & +0.18 & +3.69 & +4.34 & +10.70 & $\ldots$ & $\ldots$ & $\ldots$ & $\ldots$ \\
\hline
\end{tabular}

M1 Mass Configuration

\begin{tabular}{|c|c|c|c|c|c|c|c|c|c|c|}
\hline \multirow{2}{*}{$\begin{array}{l}\text { Booster Modes } 1 \text { through } 2 \text { used. } \\
\text { Orbiter Mode } 1 \text { used. }\end{array}$} & \multicolumn{10}{|c|}{ Mode Number } \\
\hline & 1 & 2 & 3 & 4 & 5 & 6 & 7 & 8 & 9 & 10 \\
\hline Theoretical frequency $(\mathrm{Hz})$ & 7.65 & 11.20 & 16.37 & $\ldots$ & $\ldots$ & $\therefore$ & $\because=$ & $\ldots$ & $\ldots$ & $\ldots$ \\
\hline Experimental frequency $(\mathrm{Hz})$ & 7.35 & 11.13 & 16.34 & $\ldots$ & $\ldots$ & $\cdots$ & $\ldots$ & $-\ldots$ & --- & ... \\
\hline Percent difference 4 & +4.08 & +0.63 & +0.18 & $\ldots$ & $\ldots$ & $\ldots$ & $\ldots$ & $\ldots$ & $\ldots$ & $\ldots$ \\
\hline
\end{tabular}

M2 Mass Configuration

\begin{tabular}{|c|c|c|c|c|c|c|c|c|c|c|}
\hline \multirow{2}{*}{$\begin{array}{l}\text { Boaster Modes } 1 \text { through } 6 \text { used. } \\
\text { Orbiter Modes } 1 \text { through } 5 \text { used. }\end{array}$} & \multicolumn{10}{|c|}{ Mode Number } \\
\hline & 1 & 2 & 3 & 4 & 5 & 6 & 7 & 8 & 9 & 10 \\
\hline Theoretical frequency ${ }^{1}(\mathrm{~Hz})$ & 7.68 & 11.23 & 16.22 & 26.41 & 38.30 & 50.18 & 56.35 & 88.53 & 96.89 & 161.65 \\
\hline Experimental frequency $(\mathrm{Hz})$ & 7.75 & 11.33 & 16.48 & 26.19 & 38.50 & 50.60 & 54.60 & 86.80 & 96.60 & 147.80 \\
\hline Percent difference 4 & -0.90 & .0 .88 & -1.58 & +0.84 & -0.52 & -0.83 & +3.20 & +1.99 & +0.30 & +9.37 \\
\hline
\end{tabular}

\begin{tabular}{|c|c|c|c|c|c|c|c|c|c|c|}
\hline Normalized Modal Mass & 1 & 2 & 3 & 4 & 5 & 6 & 7 & 8 & 9 & 10 \\
\hline M1 mass configuration (Kgs) & 4. 36 & 5.33 & 4.81 & 13.05 & 5.46 & 4.80 & 17.05 & 9.98 & 6.24 & 9.69 \\
\hline M2 mass configuration ( $\mathrm{Kgs}$ ) & 3.68 & 5.50 & 4.45 & 12.84 & 3.38 & 5.66 & 20.17 & 6.88 & 3.99 & 6.85 \\
\hline
\end{tabular}

Undamped eigenvalue results.

D2 damping: damped eigenvalue results.

D3 damping: damped engenvalue results.

Based on experimental frequency compared with undamped eigenvalue results. 
two Booster modes and only one Orbiter mode. The convergence of the predicted frequencies is evident as we proceed from the lower to the higher number of modes used in the analysis.

Table VIII(b) shows the natural frequencies and modal masses obtained for the free-free component synthesis for the Ml mass configuration. The highest frequency which could be predicted was for the sixth mode with poor results thereafter. This is also evident in the mode shapes obtained as shown in Figure 14a. Similar results were anticipated for the M2 mass configuration, and thus, an analysis for this configuration using free-free component modes was not made.

\section{B. Damping}

The System damping used to predict System responses was derived from the method outlined in Section VI. In addition to the damping values obtained by our method, we also considered damping values which were obtained from a complex eigenvalue solution as discussed in Section III. D. 4. These values give an indication of the amount of error which could be expected if a straightforward synthesis of damping through the coupling matrices was done. Table IX gives the System modal damping factors obtained by our method and those obtained from the eigenvalue results. These are presented for the Ll link condition only. As pointed out in Section V, component damping energy was obtained experimentally for both the pin-slip and free-free configurations. Our damping prediction method was applied to both sets of curves, and the results are presented in Table IX. Related data in terms of damping energy per cycle is presented in Figure 15 for the free-free synthesis method. Although the predicted results are shown as smooth curves, the individual predicted points deviated from the curve by about the same order of error as the experimental points did in the original component curves (Figures 8 and 12).

In general, it appears that the System damping factors obtained from the free-free damping energy curves agree with the experimental factors somewhat better than those obtained from the pin-slip energy curves. The average percent difference is of the order of \pm 15 percent for the damping factors synthesized from the free-free curves and is slightly higher for the pin-slip synthesis. This range of error is similar to that present in the original component data (Figures 8 and 12), and is an extreme improvement over those values obtained directly from the eigenvalue results, where the difference between predicted damping and experimental damping varies somewhere between 100 and 325 percent.

To obtain the additional modal damping for the various link dampers (L2, L3, L4), we simply add the predicted energy dissipated by the link damper to the predicted energy dissipated by the remaining structure. 

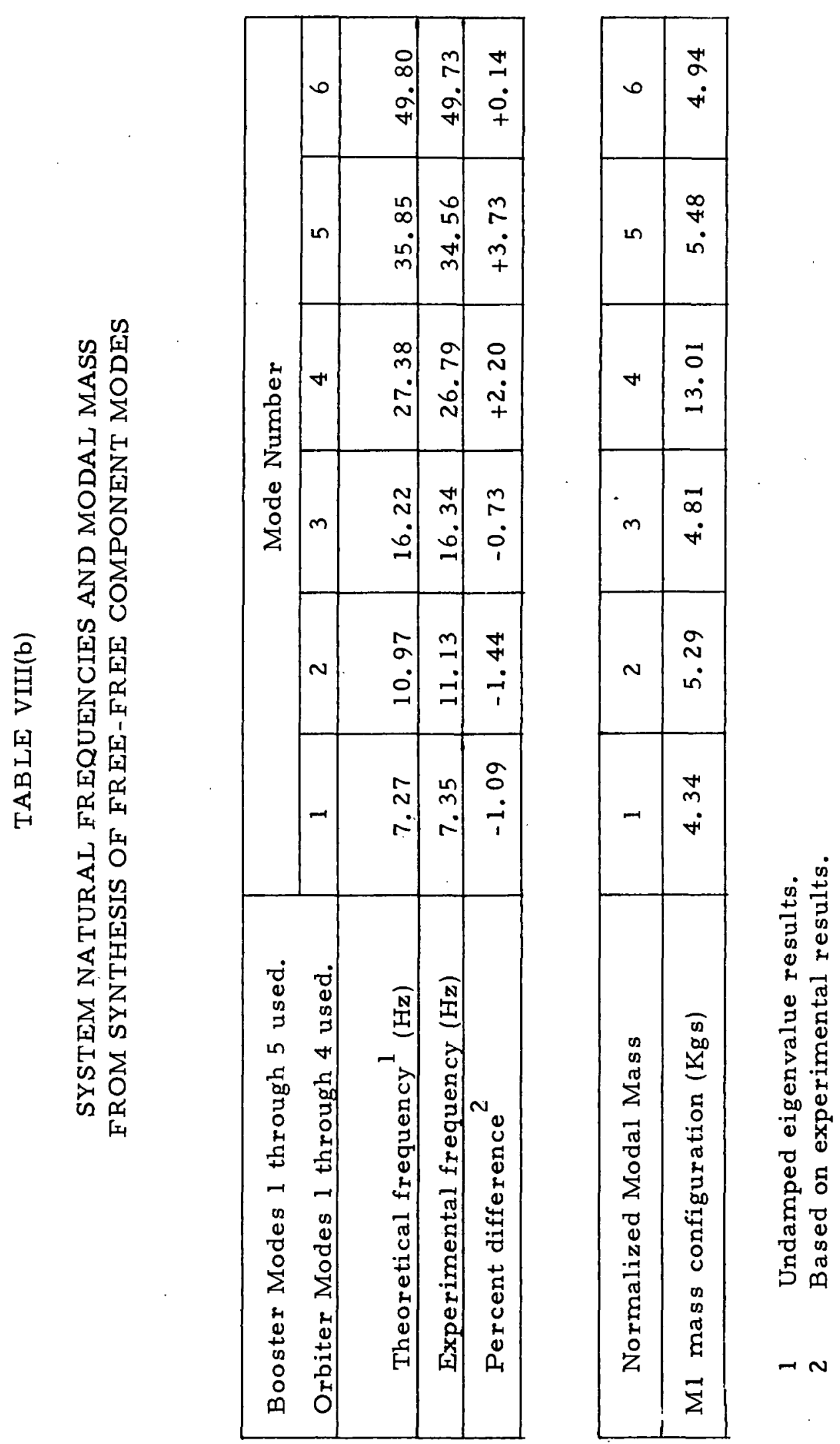
TABLE IX. SYSTEM DAMPING

(For Ll Cases only)

\begin{tabular}{|c|c|c|c|c|c|c|c|c|c|c|}
\hline \multirow{2}{*}{ S-F-L1-Dl-Ml } & \multicolumn{10}{|c|}{ Mode Number } \\
\hline & 1 & 2 & 3 & 4 & 5 & 6 & 7 & 8 & 9 & 10 \\
\hline Energy Prediction & .0071 & .0089 & .0065 & .0050 & .0049 & .0042 & $\cdots$ & $-\cdots$ & .0038 & .0035 \\
\hline Experimental Results & .0070 & 0074 & .0047 & .0046 & .0039 & 0037 & $\cdots$ & $-\ldots$ & .0028 & .0032 \\
\hline
\end{tabular}

\begin{tabular}{|c|c|c|c|c|c|c|c|c|c|c|}
\hline \multirow{2}{*}{$S-F-L 1-D 2-M 1$} & \multicolumn{10}{|c|}{ Mode Number } \\
\hline & 1 & 2 & 3 & 4 & 5 & 6 & 7 & 8 & 9 & 10 \\
\hline Energy Prediction ${ }^{l}$ & .0143 & .0128 & .0103 & .0066 & .0075 & 0066 & $\cdots$ & $\cdots$ & .0057 & .0049 \\
\hline Energy Prediction ${ }^{2}$ & .0138 & .0100 & .0088 & .0061 & .0060 & .0053 & -- & --- & .0046 & .0040 \\
\hline Eigenvalue Results & .0243 & .0135 & .0147 & .0277 & .0102 & .0099 & .0082 & .0133 & .0069 & .0103 \\
\hline Experimental Results & .0143 & .0102 & .0079 & .0071 & .0067 & .0053 & $=$ & $\ldots$ & .0043 & 0049 \\
\hline
\end{tabular}

\begin{tabular}{|c|c|c|c|c|c|c|c|c|c|c|}
\hline \multirow{2}{*}{$\mathrm{S}-\mathrm{F}-\mathrm{Ll}-\mathrm{D} 3-\mathrm{M} 1$} & \multicolumn{10}{|c|}{ Mode Number } \\
\hline & 1 & 2 & 3 & 4 & 5 & 6 & 7 & 8 & 9 & 10 \\
\hline Energy Prediction 1 & .0296 & 0237 & .0196 & .0130 & 0133 & .0112 & -- & --- & 0087 & 0078 \\
\hline Energy Prediction ${ }^{2}$ & .0330 & .0226 & .0190 & .0113 & .0120 & .0103 & --- & $\cdots$ & .0080 & .0069 \\
\hline Eigenvalue Results & .0562 & .0278 & .0311 & .0607 & .0189 & .0166 & .0133 & .0256 & .0118 & .0193 \\
\hline Experimental Results & .0332 & .0232 & .0172 & .0143 & .0115 & .0105 & -- & $\ldots$ & .0080 & .0086 \\
\hline
\end{tabular}

\begin{tabular}{|c|c|c|c|c|c|c|c|c|c|c|}
\hline \multirow{2}{*}{$\mathrm{S}-\mathrm{F}-\mathrm{L} 1-\mathrm{D} 2 / \mathrm{D} 3-\mathrm{Ml}$} & \multicolumn{10}{|c|}{ Mode Number } \\
\hline & 1 & 2 & 3 & 4 & 5 & 6 & 7 & 8 & 9 & 10 \\
\hline Energy Prediction 1 & .0169 & .0238 & .0136 & .0102 & .0088 & .0071 & -- & $\cdots$ & .0067 & .0052 \\
\hline Energy Prediction ${ }^{2}$ & .0159 & .0208 & .0113 & .0099 & .0074 & .0060 & $\cdots$ & $\ldots$ & .0059 & .0047 \\
\hline Experimental Results & .0169 & .0230 & .0106 & .0106 & .0073 & .0065 & - & $\cdots$ & .0057 & .0054 \\
\hline
\end{tabular}

\begin{tabular}{|c|c|c|c|c|c|c|c|c|c|c|}
\hline \multirow{2}{*}{$S-F-L 1-D 2-M 2$} & \multicolumn{10}{|c|}{ Mode Number } \\
\hline & 1 & 2 & 3 & 4 & 5 & 6 & 7 & 8 & 9 & 10 \\
\hline Energy Prediction 1 & .0124 & .0091 & .0080 & .0058 & .0054 & .0053 & --- & .0041 & .0044 & $\ldots$ \\
\hline Experimental Results & .0137 & .0106 & .0071 & .0071 & .0050 & .0056 & $\ldots$ & 0067 & .0056 & $\ldots$ \\
\hline
\end{tabular}

1 Using pin-slip modes

2 Using free-free modes 


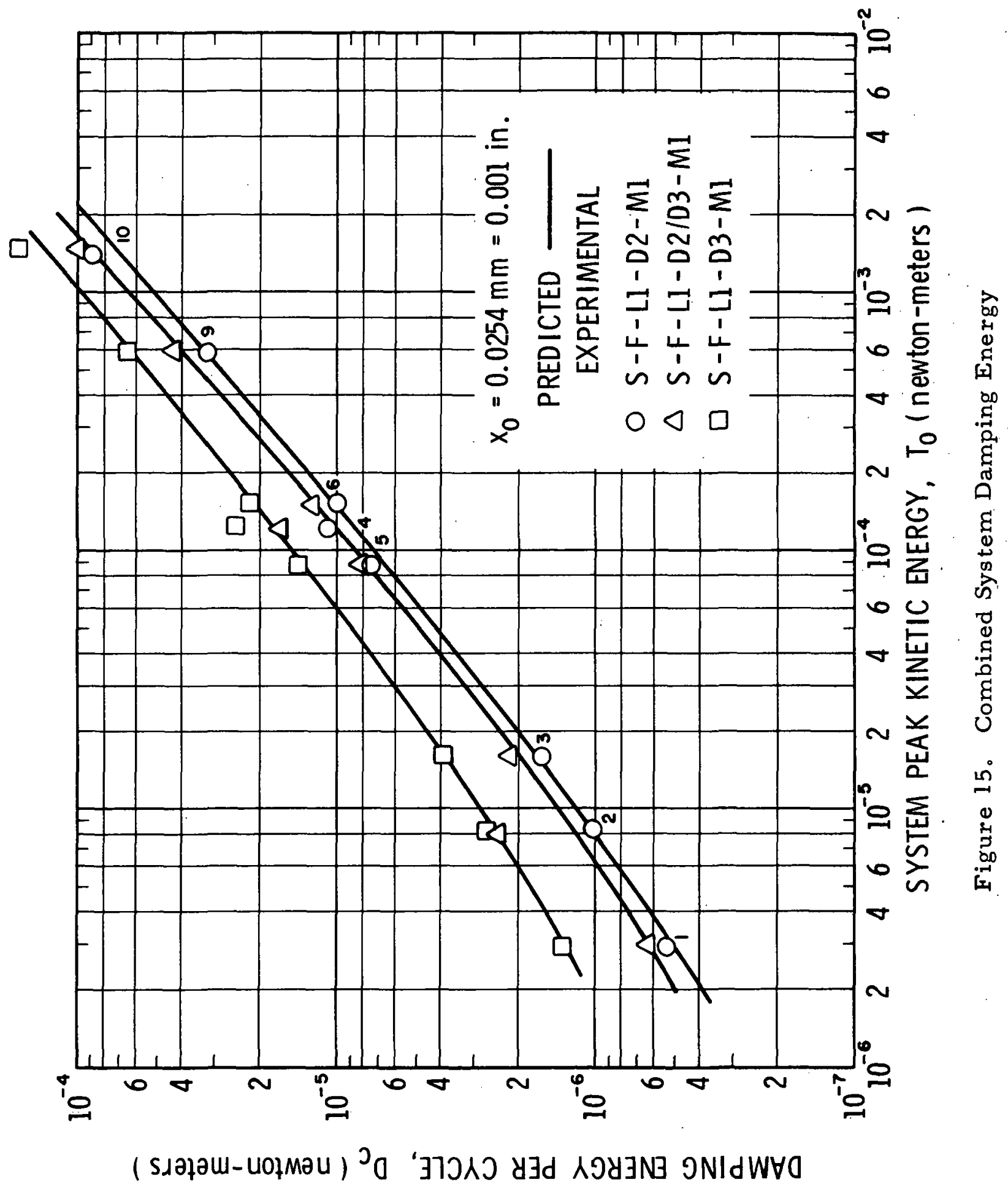


The predicted link damping energy is given by $D_{c l}=\pi C \phi_{\mathrm{rel}}^{2} \omega$, where the link damping coefficients $C$ are given in Table III and $\phi_{r e l}$ are theoretical mode shape values. Thus, the damping factor for both the structure and link damper is the sum of the individual damping factors as given by Equation (116). This also provides a further cross-check of data by comparing the sum of measured damping factors for the link acting alone and oil acting alone, to measured values obtained when both act simultaneously. Table $X$ gives the damping factors for the various structure damping configurations in combination with the various link dampers.

\section{Forced Response}

Forced response measurements were made on the Booster at node 10 and on the Orbiter at node 4 for a constant input force level at node 10 in the lateral (z) direction. Figures 16, 17, and 18 show the experimental and predicted forced responses in the form of response in DB versus frequency, similar to the procedures outlined for the component response. They are based on the use of Equation (62), with System natural modes obtained from pin-slip synthesis and modal damping given by Tables IX and X. Pin-slip, rather than free-free synthesis eigenvectors were used in the expansion of forced response, since less pin-slip synthesis System eigenvectors are required for sufficient accuracy above the sixth mode, as has been indicated in Figure 14.

The measurements were made on the S-F-L1-D2-M1 and S-F-L1-D3-M1 configurations to determine the accuracy of the mathematical model and damping prediction for two different structural damping conditions that did not include link damping. These are Figures 16 and 17 , respectively. In addition, Figure 18 shows the results obtained whereby we added the additional L3 link damping to the S-F-LI-D2-MI configuration. It should be noted in this figure that the predicted responses seem to be consistently higher than the measured responses. We suspect that this was a result of a change in the calibration of the force link used to drive the structure, but have not verified this. A comparison of the predicted responses and the measured responses from the above figures indicates that the agreement is very good to excellent over the entire frequency range and in particular at the peaks, where damping is the dominant factor.

It must be emphasized that in all forced response predictions, the predicted damping factors were used, not the experimental ones. Assuming that the mathematical model exactly duplicated the dynamic modal mass and stiffness behavior of the Shuttle model, then the only difference between predicted and experimental responses would come as a result of the differences in predicted and experimental damping. Our mathematical model, as is the case with all mathematical models, does not exactly duplicate the dynamic behavior of the Shuttle model, although judging by the frequencies and mode shapes obtained, it is a fairly good one. 

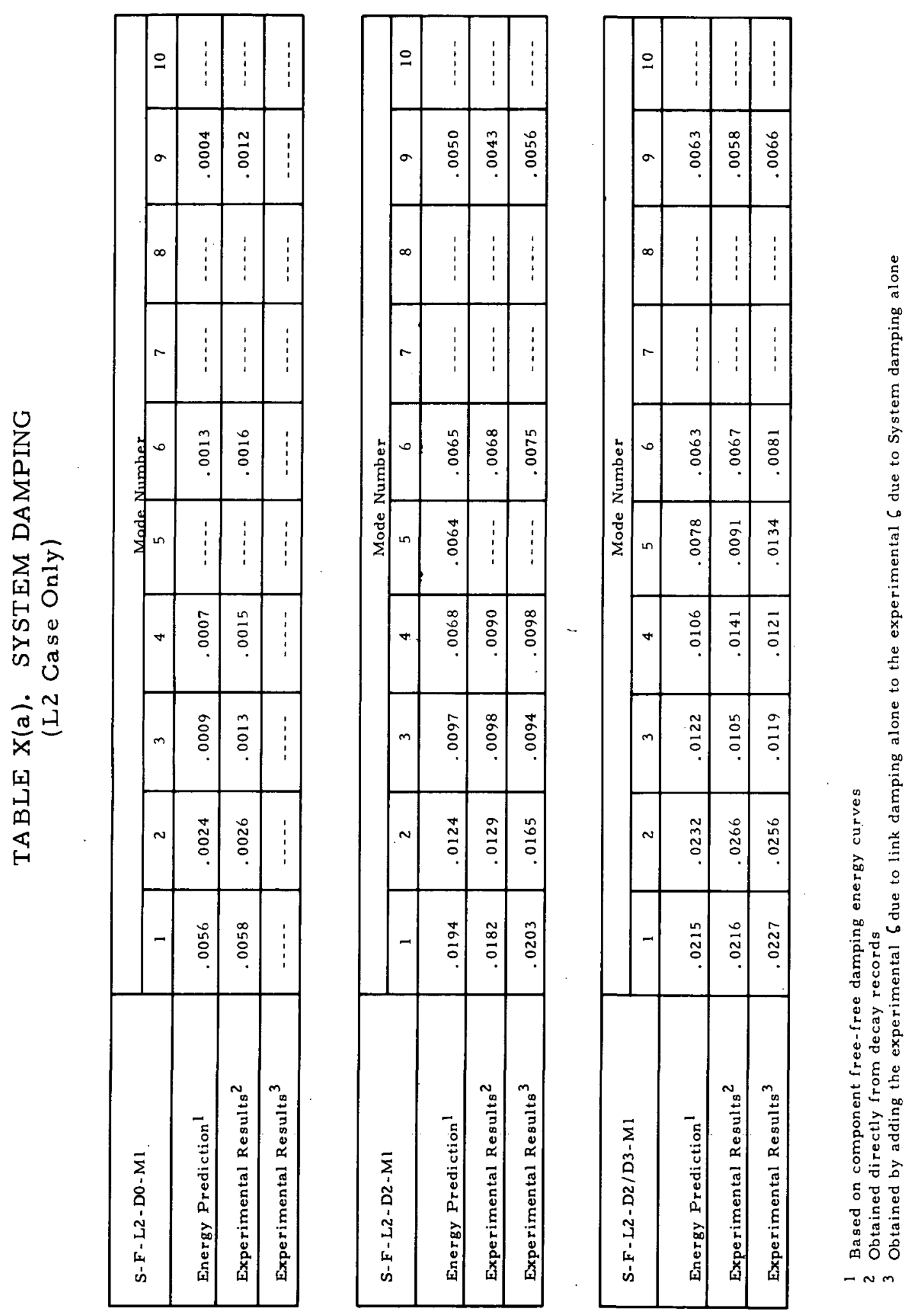

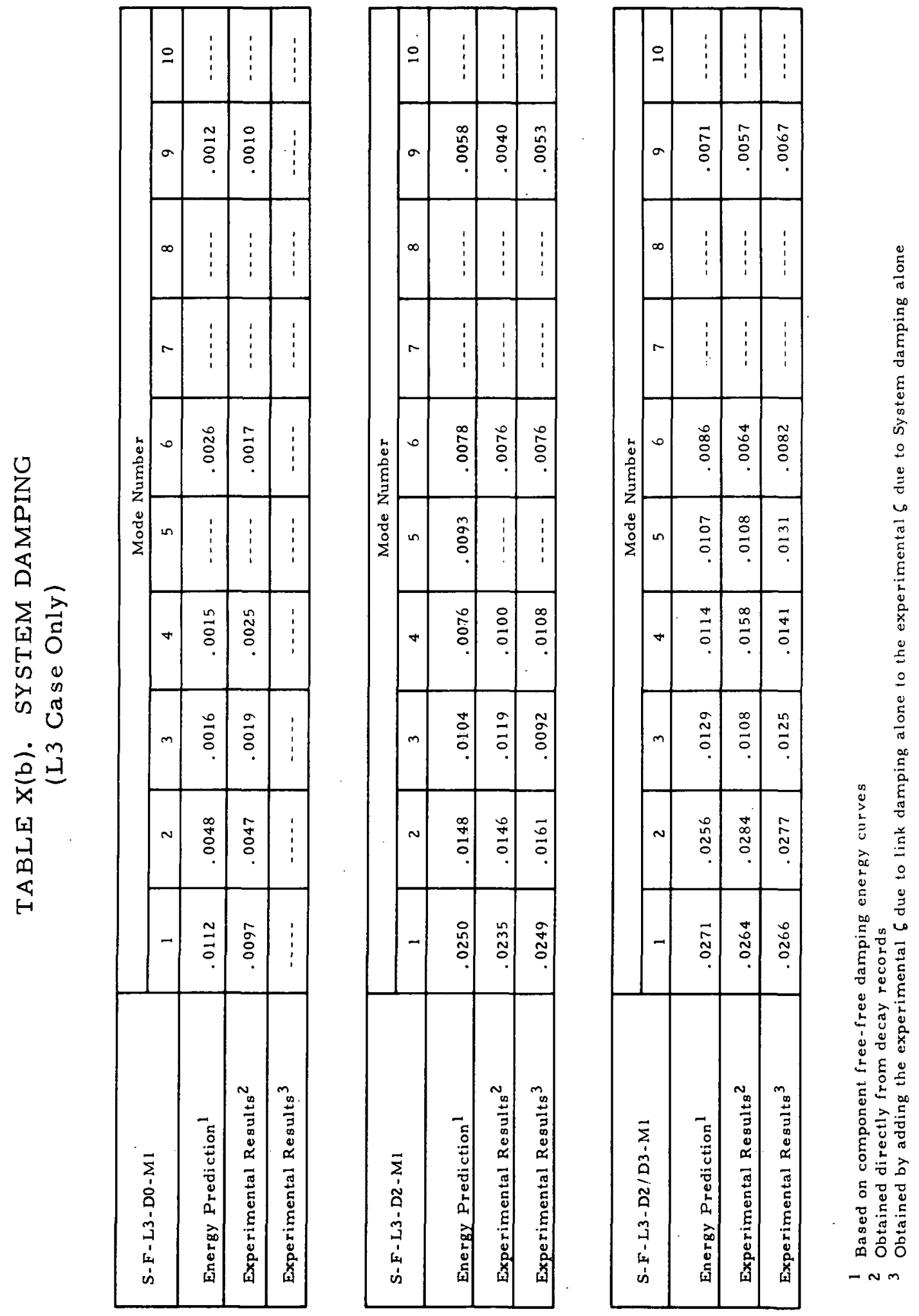

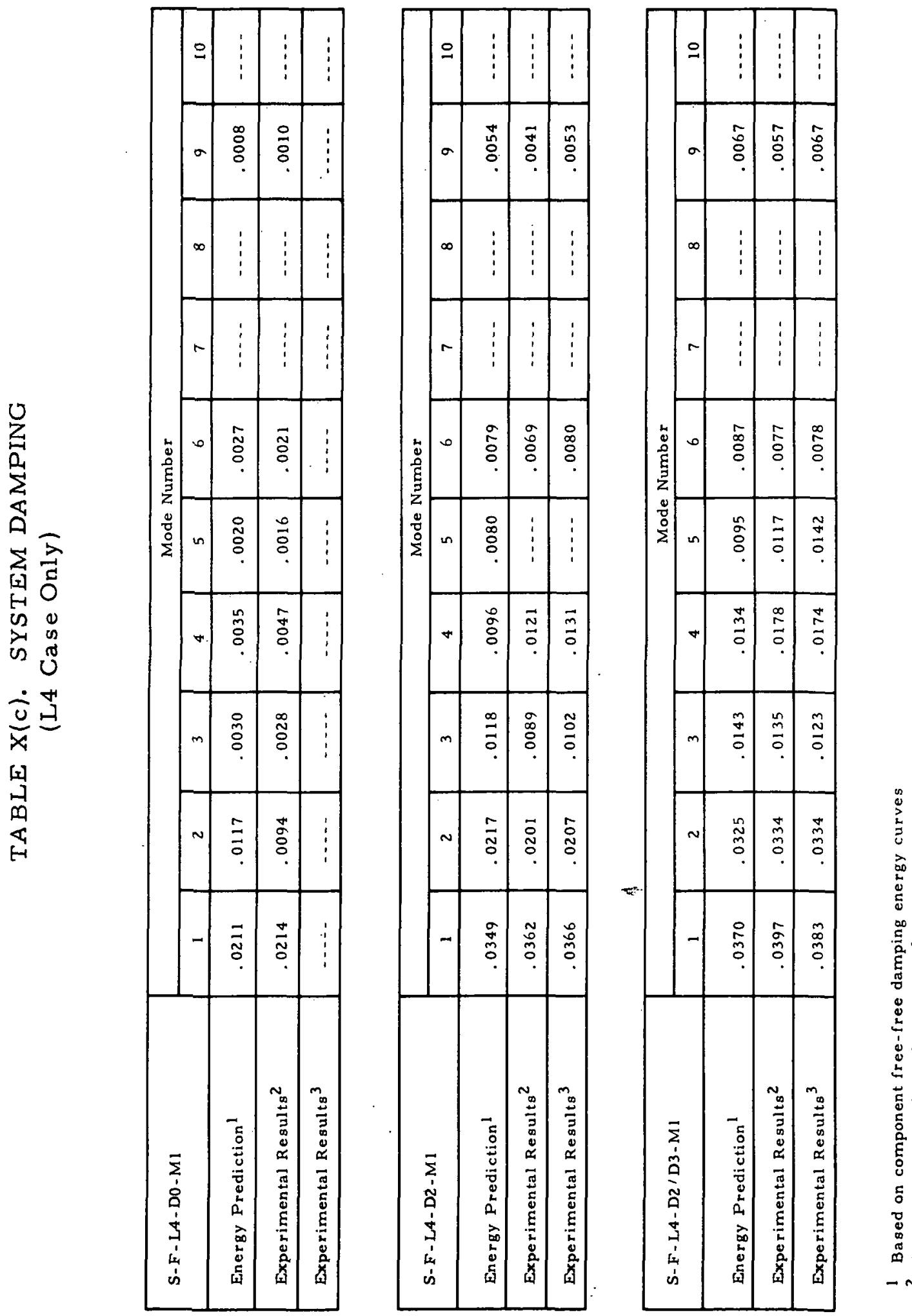

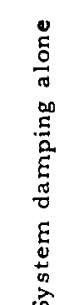
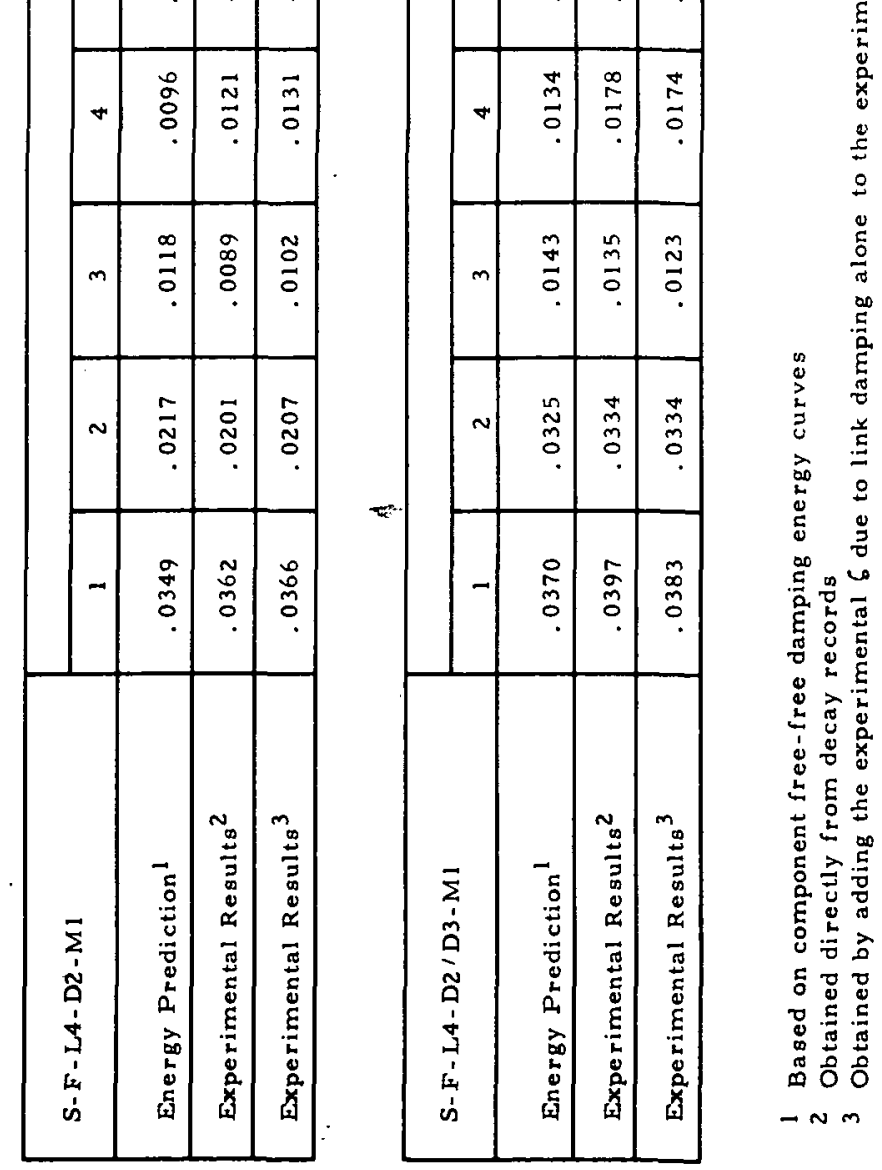


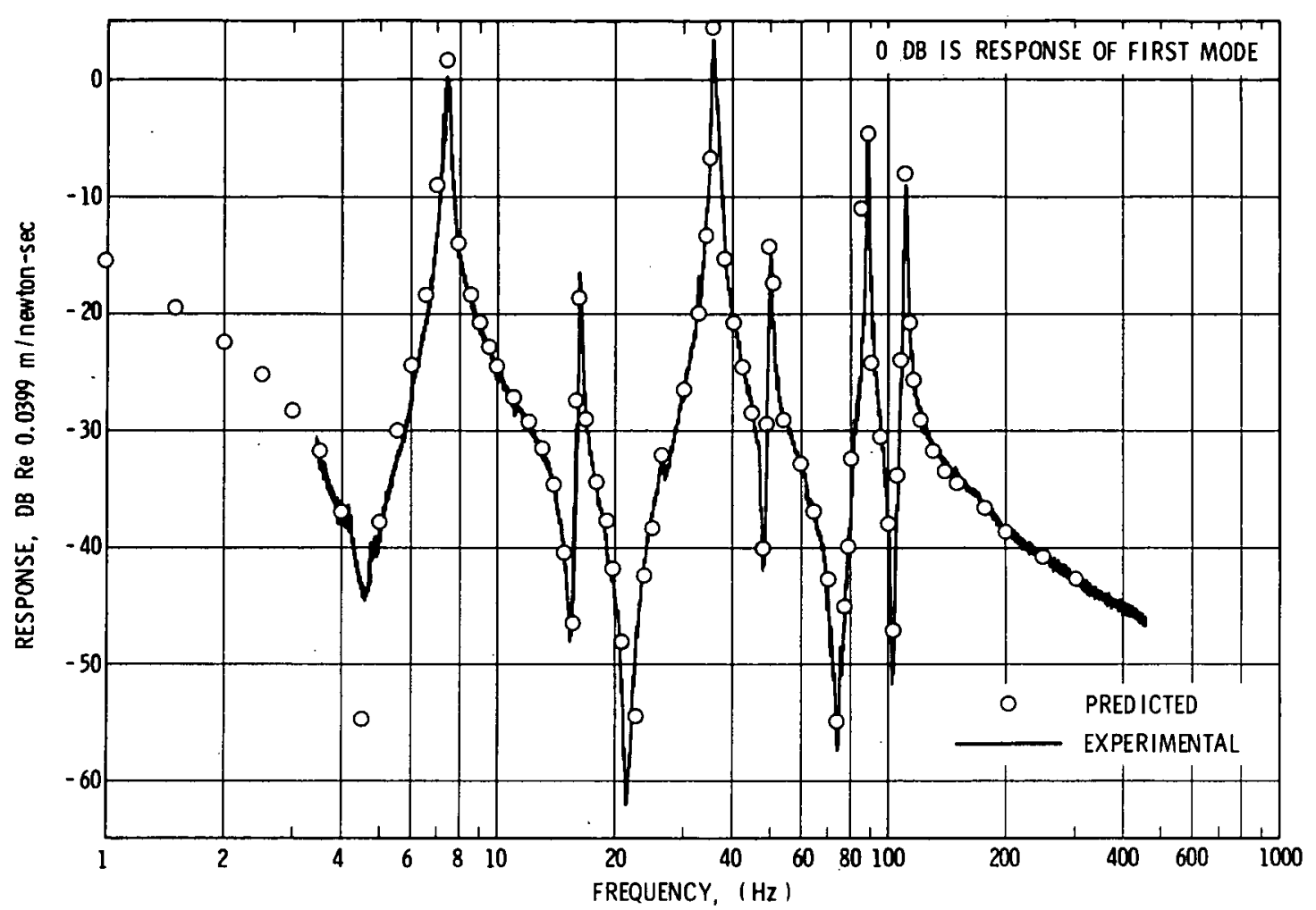

(a) Booster-Mass 10

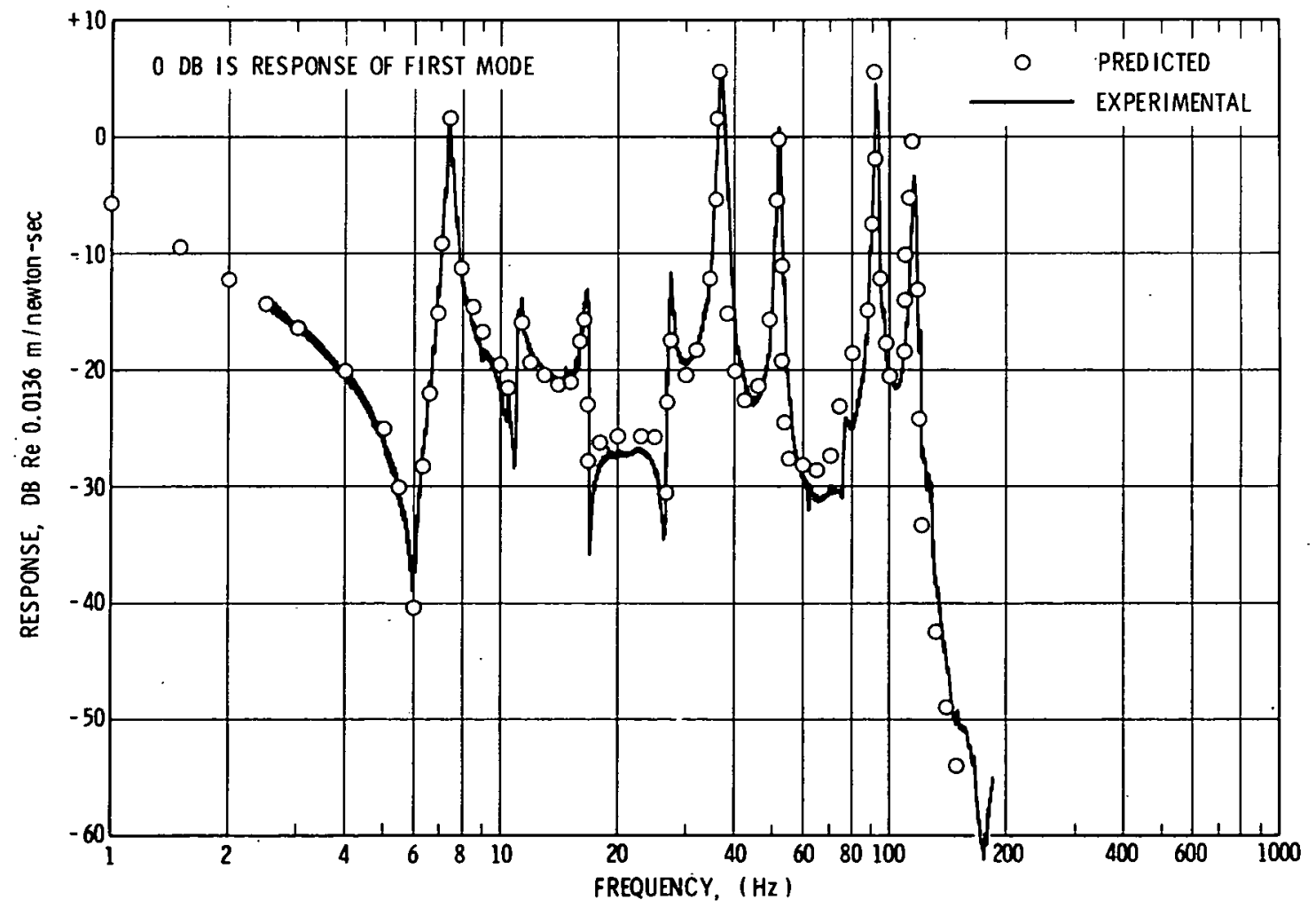

(b) Orbiter-Mass 4

Figure 16. System Response for S-F-Ll-D2-Ml Configuration 


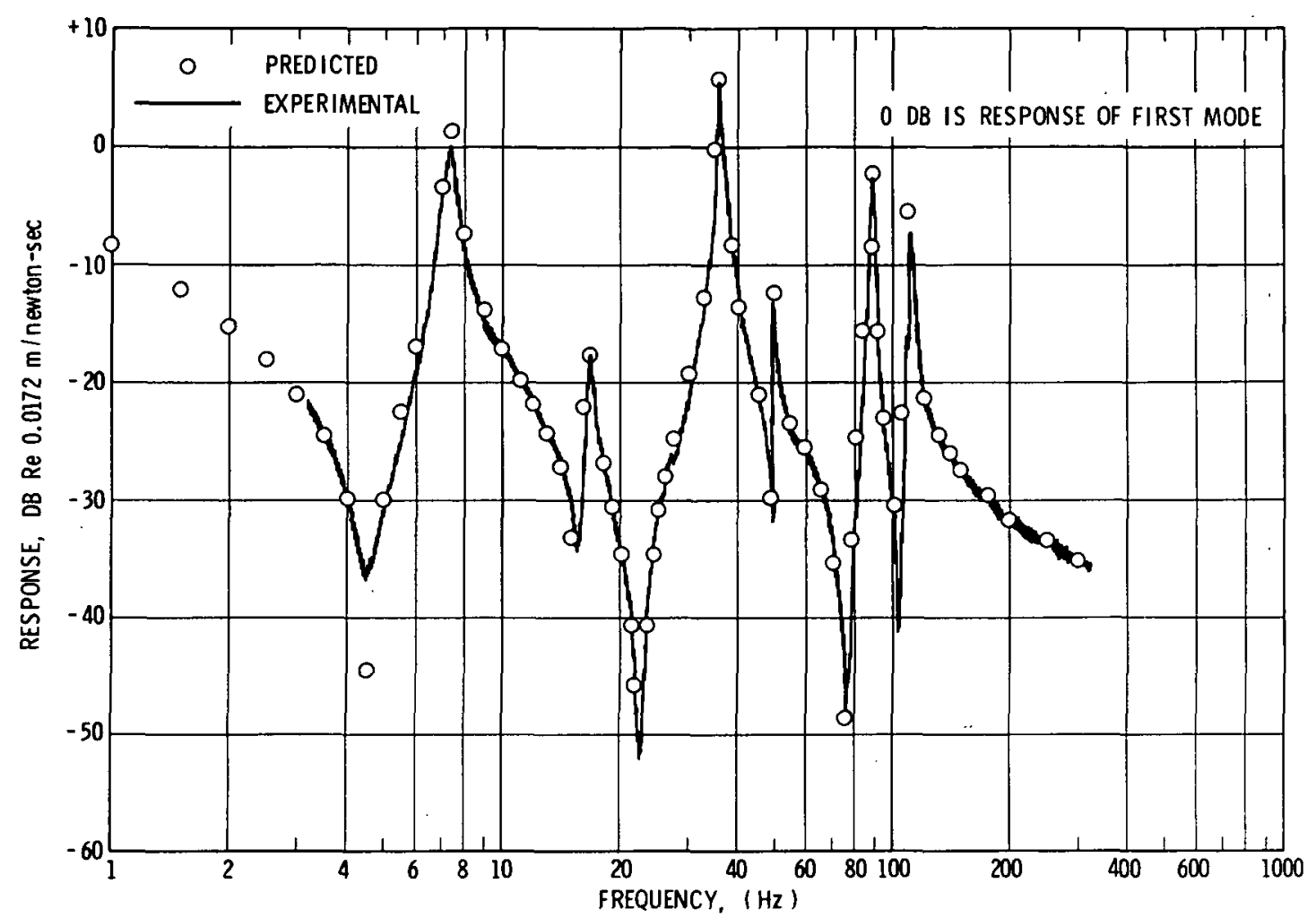

(a) Booster-Mass 10

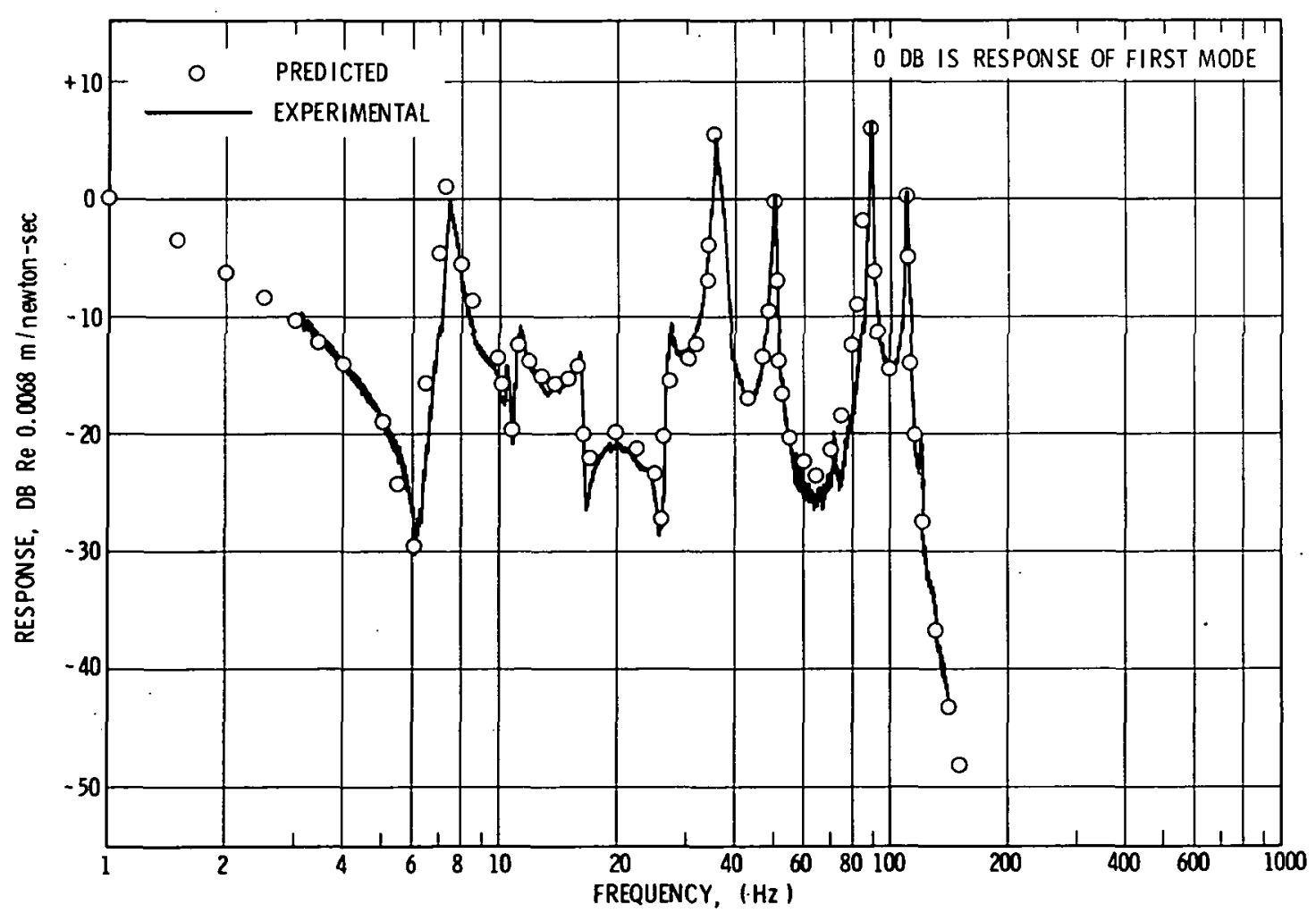

(b) Orbiter-Mass 4

Figure 17. System Response for S-F-L1-D3-M1 Configuration 

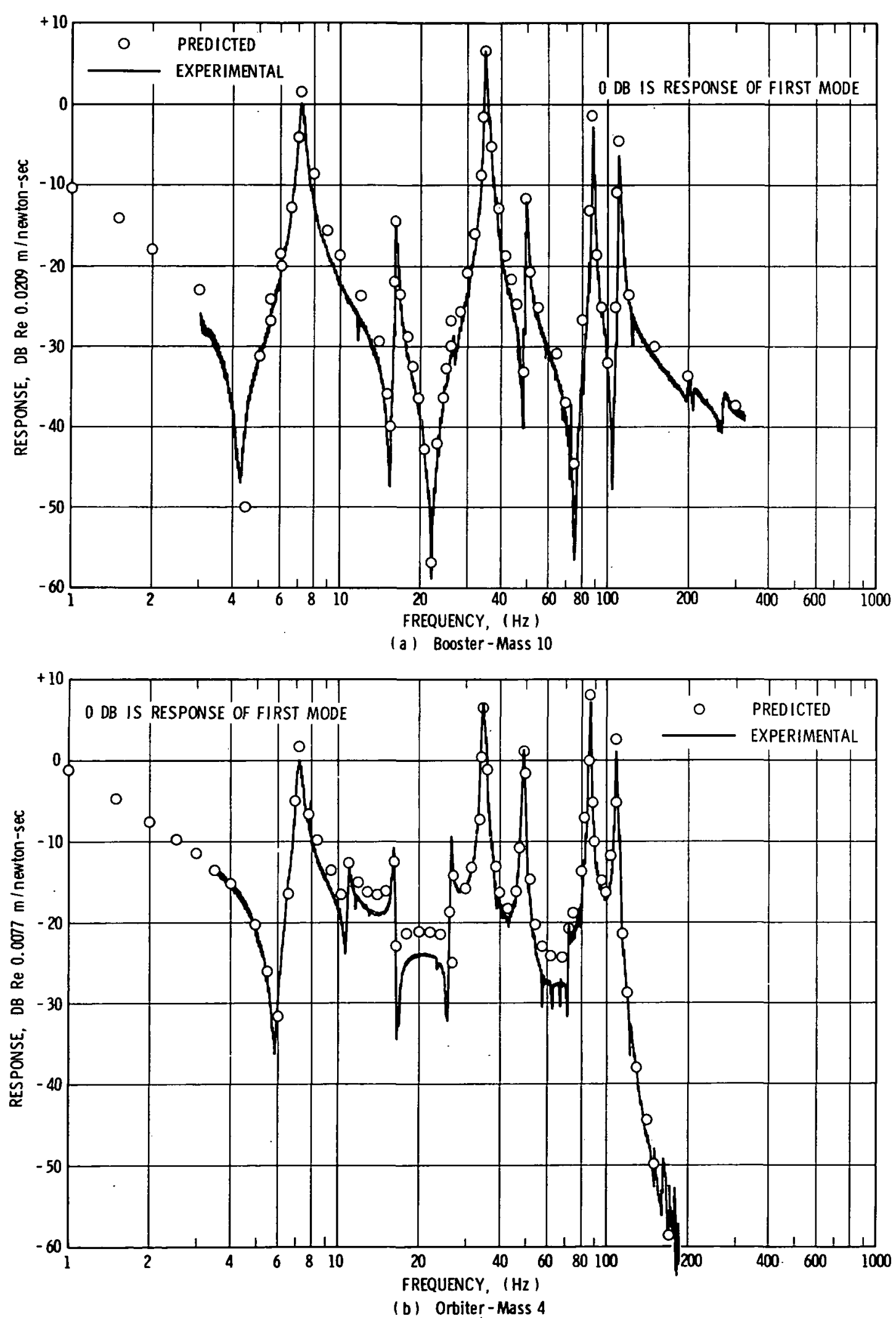

Figure 18. System Response for S-F-L3-D2-M1 Configuration 
Nevertheless, the differences we obtain between predicted and experimental responses arise now due to the differences in predicted and experimental damping, plus the differences in the mathematical and real-life Shuttle models. Considering these two factors in combination, it is believed that the System forced response results obtained in this study indicate the feasibility of substructure testing with subsequent synthesis of the component results.

\section{Verification of Damping Synthesis Hypothesis}

It has been pointed out in Section VII, that a necessary part of our damping synthesis method includes the hypothesis that experimentallydetermined curves of the form given in Figures 8 and 12 can be used to obtain appropriate damping energies for the components at kinetic energies that correspond to points intermediate to the original components. We now consider a verification of this hypothesis through the use of System damping data.

Let us consider for the moment, that our primary intent in this study was to obtain data that would verify the above hypothesis. We start with the curves as they appear in Figures 8 and 12 , the points for which have been determined by component resonance tests. As has been previously mentioned, we do not consider off-resonance testing to obtain intermediate values, because of the inherent inaccuracy associated with such measurements. Therefore, in order to obtain values at intermediate kinetic energies, and still allow the use of resonance testing, we must change the boundary conditions on the respective components. At the same time, we must preserve the essential form of damping mechanisms in the structure. This is precisely what was done in combining the components into the System tests. Therefore, we can consider our measurements of System damping, and use these data to fill in points on the original component curves. In other words, we use the data in the inverse form to which it has been considered previously.

As an example, we consider the free-free component curves in Figure 12. We wish to use the System damping results from the various configurations to fill in additional points on these curves (assuming the data will fall on the respective curves). To do this, we must first note that the System damping energies must be properly apportioned to the Booster and Orbiter, for each System mode. This is done on the basis of the amount of kinetic energy that each component contributes to the System mode, which is consistent with the conceptual basis for plotting Figures 8 and 12 . 


$$
\begin{aligned}
& \overline{\mathrm{D}}_{\mathrm{cB} \mathrm{av}}=4 \pi \overline{\mathrm{T}}_{O B} \zeta_{\mathrm{b}} \\
& \overline{\mathrm{D}}_{\mathrm{cov}_{\mathrm{av}}}=4 \pi \overline{\mathrm{T}}_{\mathrm{OO}} \zeta_{\mathrm{B}}
\end{aligned}
$$

where $\overline{\mathrm{T}}_{O B}$ and $\overline{\mathrm{T}}_{O O}$ are the respective Booster and Orbiter kinetic energies at a maximum modal amplitude of $x_{0}$, and $\zeta_{s}$ is the measured value of System damping in a given mode. Then note that we can write

$$
\begin{aligned}
& \bar{D}_{c B}=\bar{D}_{c B 1} \frac{\bar{T}_{O B}}{\bar{T}_{O S}}+\bar{D}_{c 01} \frac{T_{O O}}{\bar{T}_{O S}} \\
& \bar{D}_{c O_{a V}}=\bar{D}_{c B 2} \frac{\bar{T}_{O A 1}}{\bar{T}_{O S}}+\bar{D}_{c 02} \frac{T_{O O}}{\bar{T}_{O S}}
\end{aligned}
$$

where $\bar{D}_{C B 1,2}$ and $\bar{D}_{c 01,2}$ are the desired intermediate damping energy values corresponding to kinetic energies $\bar{T}_{O B}$ and $\bar{T}_{00}$, and both are for maximum modal amplitudes of $x_{0}$; Further, $T_{00}$ corresponds with a maximum modal amplitude of $x^{\prime}$ (we assume that $x^{\prime}<x_{0}$ for a given System mode here), and $\overline{\mathrm{T}}_{\mathrm{OS}}$ is System kinetic energy. Equations 118 provide the desired apportioning of damping energy according to the respective kinetic energies present in a given system mode, and contain four unknowns. Two more equations can be formulated from Figures $12 \mathrm{a}$ and $12 \mathrm{~b}$ as

$$
\begin{aligned}
& \overline{\mathrm{D}}_{\mathrm{c} a 1}=k_{2} \overline{\mathrm{D}}_{\mathrm{cBI}} \\
& \overline{\mathrm{D}}_{\mathrm{cBZ}}=k_{2} \overline{\mathrm{D}}_{\mathrm{c} 02}
\end{aligned}
$$

where $k_{1}$ and $k_{2}$ are the ratios of the curve values at respective kinetic energies $\overline{\mathrm{T}}_{O B}$ and $\overline{\mathrm{T}}_{O O}$.

Equations (118) and (119) can be solved to provide

$$
\begin{aligned}
& \bar{D}_{c B 1}=\frac{\bar{D}_{c B a v} \bar{T}_{O S}}{\left(\bar{T}_{O B}+k_{1} T_{O O}\right)} \\
& \bar{D}_{c 02}=\frac{\bar{D}_{c O} \bar{T}_{o v}}{\left(T_{O O}+k_{2} \bar{T}_{O B}\right)}
\end{aligned}
$$

Note that if the Booster and Orbiter component curves (Figures $12 \mathrm{a}, \mathrm{b}$ ) happen to coincide, then $k_{1}=k_{2}=1$, and Equations 120 reduce to 


$$
\begin{aligned}
& \overline{\mathrm{D}}_{c B I}=\overline{\mathrm{D}}_{c B a v} \\
& \overline{\mathrm{D}}_{c a z}=\overline{\mathrm{D}}_{c \mathrm{O}_{a v}}
\end{aligned}
$$

Also note that care must be exercised in the use of Equations (120) so that the appropriate amplitudes $x_{0}$ and $x^{\prime}$ are assigned in each respective System mode.

System data for several configurations were used in Equation (120b) in order to obtain the results shown for the Orbiter in Figure 19. Except for one or two isolated points, these data confirm conclusively the original hypothesis, within the same order of error as was present throughout the damping measurements. It is particularly noteworthy that the data for the D2/D3 configuration also fell on the desired curves, since the Booster and Orbiter damping characteristics are more significantly different for this configuration. A plot similar to Figure 19 could also have been produced for the Booster, and comparisons with pin-slip configurations could also be employed. However, Figure 19 should be sufficient to make our point. 


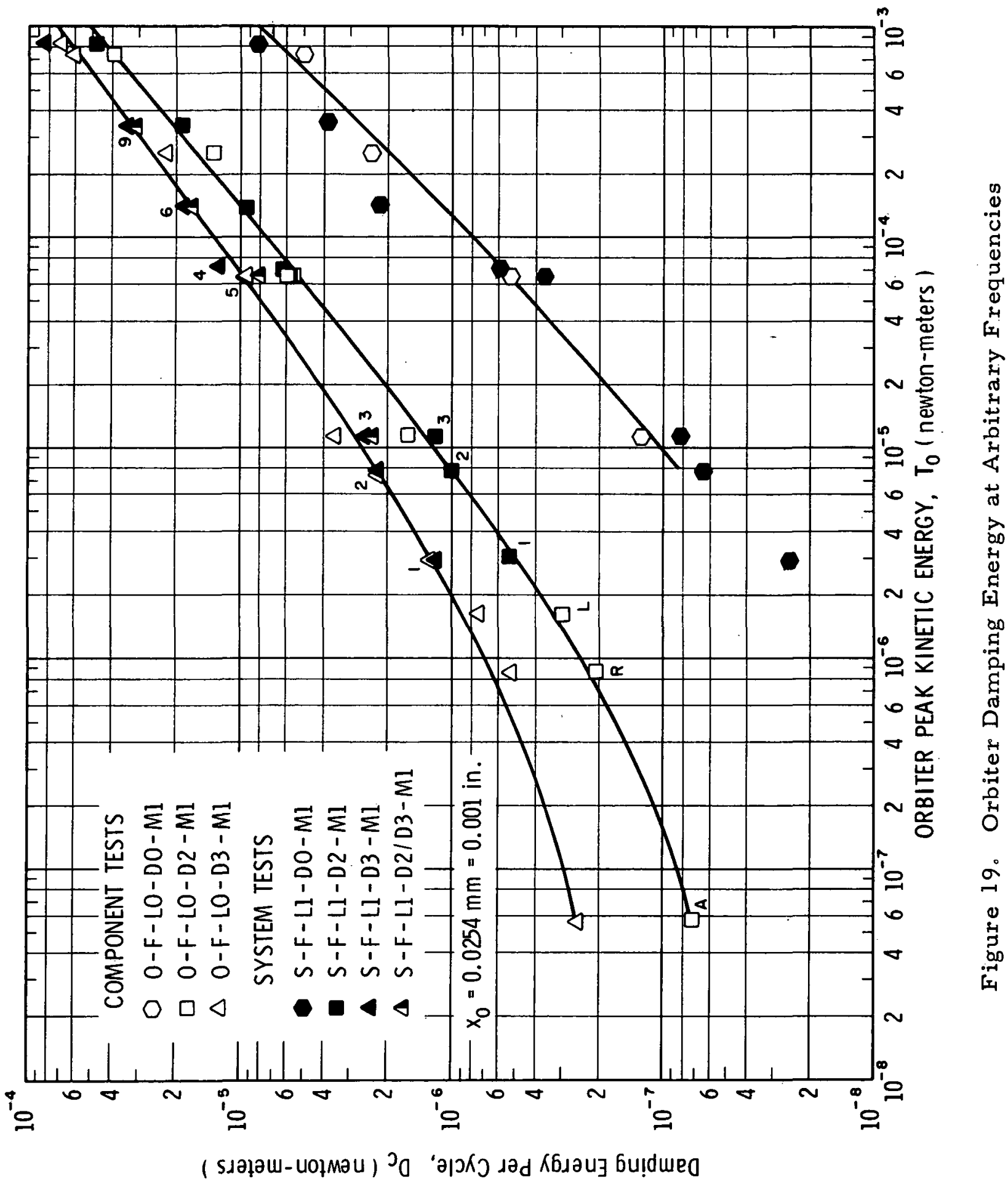




\section{FINAL DISCUSSION}

The results of this study have quite dramatically shown the successful development of a method for predicting damping in combined structural systems with accuracy that is commensurate with that of original measurements made on the substructures, for a relatively simple parallel-stage Shuttle model. The application of this method to a more representative model, and for that matter to a prototype, must still be done with caution, and some comments in this regard will be given shortly.

It is first appropriate to discuss the significance of the form of the damping energy results in Figures 8 and 12. In essence, each curve represents an aggregate or spatial integration of all of the complex damping processes which occur in the structure. No assumption is made about the particular form of damping present, except that linearity with amplitude was found to be present in these experiments. At the same time nonlinearity with kinetic energy level exists. We note that the curves for pin-slip configurations in Figures $8 \mathrm{a}, \mathrm{b}$ a re somewhat different than those for free-free conditions in Figures $12 a, b$, for corresponding mass and damping conditions. In effect, this indicates a significant redistribution of the damping processes for the different boundary conditions. It was determined that this difference results primarily from the presence of longitudinal motion in all pin-slip modes, and the form of damping chosen, which provided higher effective damping for motion in that direction. Of course, this same damping mechanism influenced the free-free modes only through rotational displacements, since the damping reservoirs were located off the longitudinal axis of the moclels, and only one (Rigid-Body) orthogonal free-free mode includes longitudinal displacements. At the same time, it appears that the basic component motions (as far as influence damping) in the System modes are more like those of the free-free component modes, rather than the pin-slip modes, since better damping predictions are provided by free-free mode synthesis. A more careful investigation of various modal displacements would also shed further light on this behavior.

The separation of results into groups representing a given aggregate damping process may cause complications in the analysis of a more complex structure. For example, certain kinds of modes may form one group (or line on a plot) while other modes may form another line. Some evidence of this possibility is indicated in the SAD- 6 data shown in Figure 20, which is based on results of experiments given in Reference 8 . The data are plotted here for fixed amplitude, and as a result, form a 


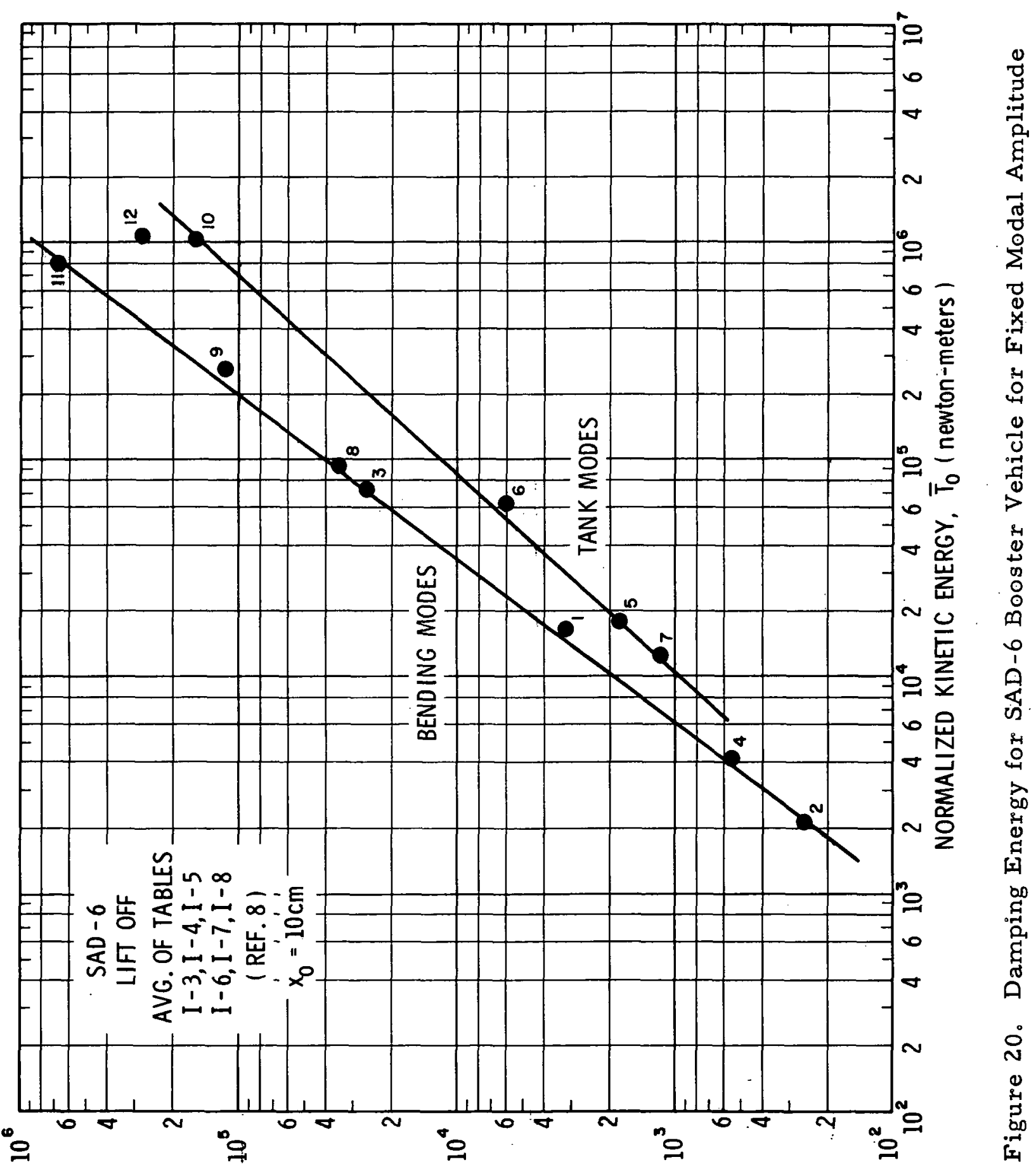

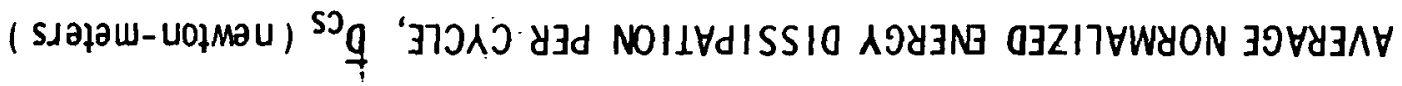


considerable rearrangement from the form in which they appeared in Reference 8 . Further, values were obtained by averaging the multiple results of modal mass obtained from tests with different suspension systems. Also mode numbers are indicated in order of increasing natural frequency. We recognize that caution must be exercised in interpreting the results as presented here. However, it appears that two basic curves are formed at the same modal amplitude, one corresponding to overall bending modes, and the other to dominantly tank cluster motion. Such multiple curves may very well occur in the even more complex Shuttle component configurations, and some means of handling them will have to be developed. For that matter, some uncertainty exists as to what degree grouping of the modes will occur. Only analysis and testing of a more representative model, which will be accomplished in the extension to the current program, can answer these important questions.

The matter of damping nonlinearity with response amplitude has been mentioned in a previous section. The concept of plotting damping energy per cycle versus peak kinetic energy is entirely amenable to such a complication. So also is the method of damping prediction developed herein. However, considerable effort will be required in additional testing in order to determine its influence, and it appears that at best, much judgement will have to be exercised to include its effects in any prototype tests. Such complications may also be the subject of extended work.

It should finally be recognized that the results of this study form a far more basic discovery than just prediction of damping in combined structures. There is the definite implication that damping energy plots can be used to identify similarities in various different complex structures. In particular, the slopes, shape, curvature, etc. may eventually be cataloged to identify damping processes in many structural applications. Thus, each curve is a plot of some now only empirical damping law, and the identification of parameters with which these laws can be described is an important new development in a very old, yet relatively little understood, subject in dynamics. 


\section{REFERENCES}

1. Kiefling, L., and Pack, H., "Structural Damping in Saturn Vehicles and Scale Models, "NASA Technical Memorandum, TM X-64607, July 8, 1971.

2. Hurty, W. C., "Dynamic Analysis of Structural Systems by Component Mode Synthesis," Technical Report No. 32-530, Jet Propulsion Laboratory, California Institute of Technology, January 15, 1964.

3. Forsberg, K. J., "Assessment of Current Methods for Dynamic Analysis of Complex Structures, "NASA TM X-52876, Vol. II, pp. 4-27, July 1970.

4. Lazan, B. J., Damping of Materials and Members in Structural Mechanics, Pergamon Press, 1968.

5. Snowdon, J. C., Vibration and Shock in Damped Mechanical Systems, John Wiley \& Sons. Inc., New York, 1968.

6. Steidel, R. F., Jr., An Introduction to Mechanical Vibrations, John Wiley \& Sons, Inc., New York, 1971.

7. Foss, K. A., "Coordinates Which Uncouple the Equations of Motion of Damped Linear Dynamics Systems, "Jour. of Applied Mechanics, Paper No. 57-A-86, 1957.

8. Chang, C., "Damping in Multi-Beam Vibration Analyses, Part IIDamping Properties of Saturn I Dynamic Test Vehicle SAD-6, " Lockheed Missiles \& Space Company Technical Memorandum TM 54-20-12, November 1964. 


\section{ACKNOWLEDGEMENTS}

The authors wish to express their sincere gratitude to several members of the Department of Mechanical Sciences who were instrumental in the successful completion of this program. Mr. Guido E. Ransleben aided in the mechanical design of the apparatus. Messrs. James J. Kulesz and George W. Downey, Jr. performed the numerous experiments, the report manuscript was typed by Mrs. Adeline K. Raeke, and all illustrations were prepared by Mr. Victor J. Hernandez. 
APPENDIX A

VALUES FOR ELEMENTS OF DISCRETE MODELS 
Orbiter Mass, $\mathrm{M}_{1}$ (Kgs)

$$
\begin{aligned}
& M_{1}=1.83 \\
& M_{2}=1.72 \\
& M_{3}=1.83 \\
& M_{4}=1.83 \\
& M_{5}=1.40 \\
& M_{6}=1.83
\end{aligned}
$$

Orbiter Moment of Inertia, $\mathrm{I}_{1}\left(\mathrm{Kg} \mathrm{s}-\mathrm{cm}^{2}\right)$

$$
\begin{aligned}
& I_{1}=28.78 \\
& I_{2}=24.84 \\
& I_{3}=28.78 \\
& I_{4}=28.78 \\
& I_{5}=19.20 \\
& I_{6}=28.78
\end{aligned}
$$

Booster Mass, $M_{1}$ (Kgs)

$$
\begin{aligned}
& M_{7}=1.83 \\
& M_{8}=1.72 \\
& M_{8}=1.83 \\
& M_{10}=1.83 \\
& M_{11}=1.40 \\
& M_{12}=1.83 \\
& M_{13}=1.83
\end{aligned}
$$

Booster Moment of Inertia, $I_{1}\left(\mathrm{Kgs}-\mathrm{cm}^{2}\right)$

$$
\begin{aligned}
& I_{7}=28.78 \\
& I_{8}=24.84 \\
& I_{8}=28.78 \\
& I_{10}=28.78 \\
& I_{11}=19.20 \\
& I_{12}=28.78 \\
& I_{13}=28.78
\end{aligned}
$$

Tube thickness, $t=0.089 \mathrm{~cm}$

Tube radius, $R=1.18 \mathrm{~cm}$

Modulus of elasticity, $E=7.30 \times 10^{6} \mathrm{Newton} / \mathrm{cm}^{2}\left(10.6 \times 10^{6} \mathrm{lb} / \mathrm{in}^{2}\right)$ Poisson's ratio, $\nu=0.325$

$\delta_{1}=8.25 \mathrm{~cm} \quad \delta_{2}=2.54 \mathrm{~cm} \quad \delta_{3}=11.43 \mathrm{~cm}$ 
Distance Between Nodes, $\ell_{1, j}(\mathrm{~cm})-$ Ml Configuration

Orbiter

$$
\begin{aligned}
& \ell_{1,2}=24.76 \\
& \ell_{2,3}=25.40 \\
& \ell_{3,4}=25.40 \\
& \ell_{4,5}=25.40 \\
& \ell_{5,6}=24.76
\end{aligned}
$$

\section{Booster}

$$
\begin{aligned}
& \ell_{y, 8}=25.40 \\
& \ell_{8,8}=25.40 \\
& \ell_{8,10}=25.40 \\
& \ell_{10,11}=25.40 \\
& \ell_{11,12}=25.40 \\
& \ell_{12,13}=24.13
\end{aligned}
$$

Distance Between Nodes, $\ell_{1,1}(\mathrm{~cm})-M 2$ Configuration

Orbiter

$$
\begin{aligned}
& \ell_{1,2}=24.76 \\
& l_{2,3}=15.24 \\
& l_{3,4}=30.48 \\
& l_{4,5}=30.48 \\
& l_{5,6}=24.76
\end{aligned}
$$

Booster

$$
\begin{aligned}
& \ell_{7,8}=25.40 \\
& \ell_{8,9}=10.16 \\
& \ell_{9,10}=30.48 \\
& \ell_{10,11}=35.56 \\
& \ell_{11,12}=15.24 \\
& \ell_{12,13}=34.29
\end{aligned}
$$


APPENDIX B

DERIVATION OF ELEMENT STIFFNESS MATRIX 
The derivation of the stiffness matrix for a beam element of length $\ell_{1, j}$ is based on Castigliano's First Theorem. If a structure is subjected to a set of external forces $P_{1}, P_{2}, \ldots P_{n}$ having displacements $u_{1}, u_{2}, \ldots$ $u_{n}$ in the direction of these forces, then by the principle of virtual work the $r$-th force may be given by

$$
P_{r}=\frac{\partial U}{\partial u_{r}}
$$

where $U$ is the strain energy of the structure. If we now take a partial derivative with respect to displacement $u_{a}$ then the stiffness coeffieicnt $k_{8 s}$ is given by

$$
k_{r s}=\frac{\partial P_{r}}{\partial u_{s}}=\frac{\partial^{2} U}{\partial u_{r} \partial u_{s}}
$$

To derive the stiffness matrix for the beam element we first express the strain energy in terms of nodal displacement $u_{1}, u_{2}, \ldots u_{6}$ as shown in Figure B-1

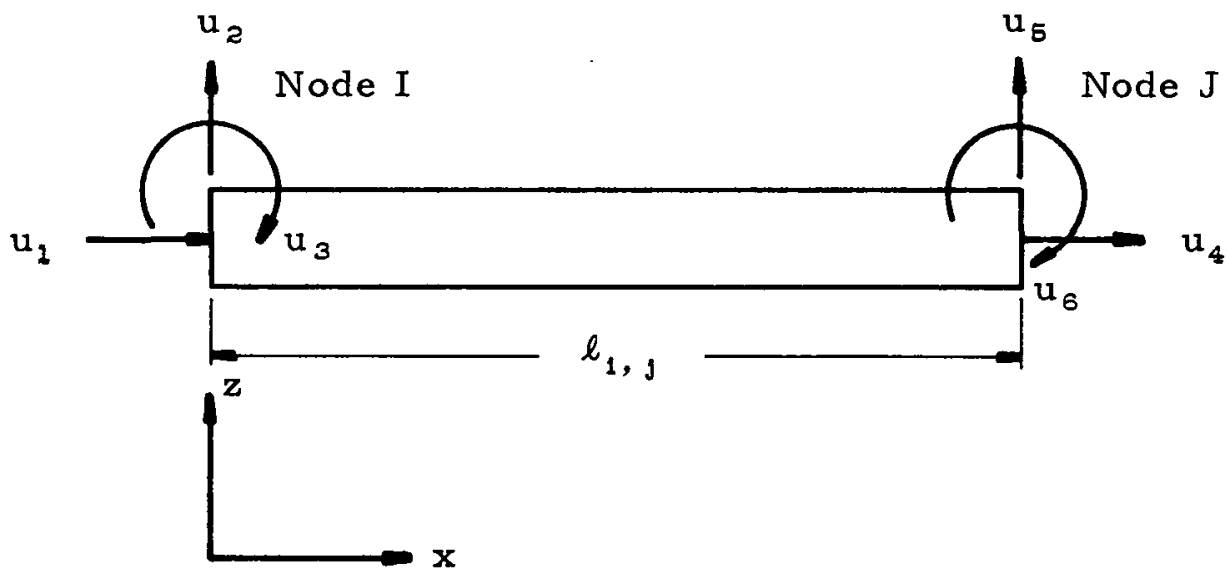

Figure B-1. Nodal Displacements of Beam Element I, J

Following the sign convention shown in Figure B-1, we may write the following shear, moment, and slope relationships:

$$
\begin{aligned}
& \frac{d V}{d x}=0 \\
& \frac{d M}{d x}=V
\end{aligned}
$$




$$
\begin{gathered}
\frac{d \phi}{d x}=\frac{M}{E d} \\
\frac{d u_{z}}{d x}=-\phi+\frac{V}{\pi R t G}
\end{gathered}
$$

where

$$
\begin{aligned}
& \mathrm{V}=\text { net shearing force on a cross-section } \\
& \mathrm{M}=\text { net moment on a cross-section } \\
& \phi \quad=\text { measure of net rotation } \\
& \mathrm{E}=\text { modulus of elasticity } \\
& \mathrm{G}=\text { shear modulus } \\
& \theta \quad=\text { moment of inertia of tube cross-section } \\
& \mathrm{R}=\text { mean radius of tube } \\
& \mathbf{t}=\text { thickness of tube wall } \\
& \mathrm{u}_{\mathbf{Z}}=\text { displacement in } z \text {-direction }
\end{aligned}
$$

be written

In the axial direction, the displacement $u_{x}$ within the element may

$$
u_{x}=\alpha_{1}+\alpha_{2} \mathbf{x}
$$

The coefficients $\alpha_{1}$ and $\alpha_{2}$ may be found by setting $u_{x}=u_{1}$ at $x=0$ and $u_{x}=u_{d}$ at $x=l_{1, j}$ from which we obtain

$$
u_{x}=u_{1}+\left(u_{4}-u_{1}\right) \frac{x}{\ell_{1, j}}
$$

The strain energy due to axial displacement is given by

$$
\mathrm{U}_{\mathrm{a}}=\frac{\mathrm{EA}}{2} \int_{0}^{\ell_{1, j}}\left(\frac{\partial \mathrm{u}_{\mathrm{x}}}{\partial \mathrm{x}}\right)^{2} \mathrm{dx}
$$

where $A=2 \pi R t$ is the tube cross-section area. Using Equations (B-8) and $(B-9)$ we obtain the stiffness coefficients from

$$
k_{r s}=\frac{\partial^{2} U_{s}}{\partial u_{r} \partial u_{s}} \quad \begin{aligned}
& r=1,4 \\
& s=1,4
\end{aligned}
$$

These stiffness coefficients are given in the element stiffness matrix, Equation (B-24). 
In the lateral direction we assume a displacement $u_{z}$ in the form

$$
u_{2}=\alpha_{3}+\alpha_{4} x+\alpha_{5} x^{2}+\alpha_{6} x^{3}
$$

The coefficients $\alpha_{3}, \alpha_{4}, \alpha_{5}$, and $\alpha_{6}$ may be found from the condition that at $\mathbf{x}=0$

$$
u_{z}=u_{2} ; \quad d u_{z} / d x=-u_{3}+\frac{V(0)}{\pi R t G}
$$

and that at $x=\ell_{1, j}$

$$
u_{z}=u_{b} ; \quad d u_{z} / d x=-u_{6}+\frac{v\left(\ell_{1, j}\right)}{\pi R t G}
$$

After some manipulation and using Equations (B-4) and (B-5) we find that $u_{z}$ can be written as

$$
\begin{aligned}
& u_{2}=u_{2}-\left[u_{3}-\frac{\left(1-B_{1,1}\right)}{l_{1, j} N_{1, j}}\left\{2\left(u_{5}-u_{2}\right)+l_{1,1}\left(u_{3}+u_{6}\right)\right\}\right] x \\
& +\frac{1}{\ell_{1,1}^{2} N_{1,1}}\left[3\left(u_{5}-u_{2}\right)+l_{1,9}\left(2 A_{1,9} u_{3}+B_{1,9} u_{6}\right)\right] x^{2} \\
& -\frac{1}{\ell_{1, j}^{3} N_{1,9}}\left[2\left(u_{5}-u_{2}\right)+\ell_{1, j}\left(u_{3}+u_{8}\right)\right] x^{3}
\end{aligned}
$$

where

$$
\begin{aligned}
& A_{1,9}=1+\frac{3 E \theta}{l_{1, j}^{2} \pi R t G} \\
& B_{1,9}=1-\frac{6 E \theta}{l_{1, j}^{2} \pi R t G} \\
& N_{1,9}=1+\frac{12 E \theta}{l_{1,9}^{2} \pi R t G}
\end{aligned}
$$

The strain energy due to bending is given by

$$
U_{b}=\frac{E d}{2} \int_{0}^{\ell_{1,1}}\left(\frac{d 2 u_{z}}{d x^{2}}\right)^{2} d x
$$

while the strain energy due to shear is given by 


$$
U_{B}=\frac{1}{2} \int_{0}^{\ell_{1, g}} \lambda \Psi 2 \mathrm{dx}
$$

where

$$
\begin{aligned}
& \lambda=\pi \text { RtG } \\
& \Psi=\text { slope due to shear }(V / \lambda)
\end{aligned}
$$

Using Equations (B-4), (B-5), and (B-6) we get

$$
V=-E \theta \frac{d^{3} u_{z}}{d x^{3}}
$$

which we substitute into Equation (B-19) to get

$$
\mathrm{U}_{0}=\frac{18 \mathrm{E}^{2} \mathrm{l}^{3}}{\lambda} \int_{0}^{\ell_{1,1}}\left(\frac{\mathrm{d}^{3} \mathrm{u}_{\mathrm{l}}}{\mathrm{d \textrm {x } ^ { 3 }}}\right)^{2} \mathrm{~d} \mathbf{x}
$$

The total strain energy due to bending and shear is given by

$$
U_{b s}=U_{b}+U_{B}=\frac{E \theta}{2} \int_{0}^{\ell_{1, g}}\left(\frac{d^{2} u_{2}}{d x^{2}}\right)^{2} d x+\frac{18 E^{2} \theta^{2}}{\lambda} \int_{0}^{\ell_{1, j}}\left(\frac{d^{3} u_{z}}{d x^{3}}\right)^{2} d x
$$

From Equation (B-14)we have $u_{z}$ which we use in obtaining $U_{b s}$. The stiffness coefficients for bending and shear are now

$$
\mathbf{k}_{r s}=\frac{\partial^{2} U_{b_{B}}}{\partial u_{s} \partial u_{s}} \quad \begin{aligned}
\mathbf{r}=2,3,5,6 \\
\mathbf{s}=2,3,5,6
\end{aligned}
$$

The required stiffness matrix may now be written as

$$
\begin{aligned}
& {\left[\mathrm{k}_{\mathrm{rB}}\right]=}
\end{aligned}
$$

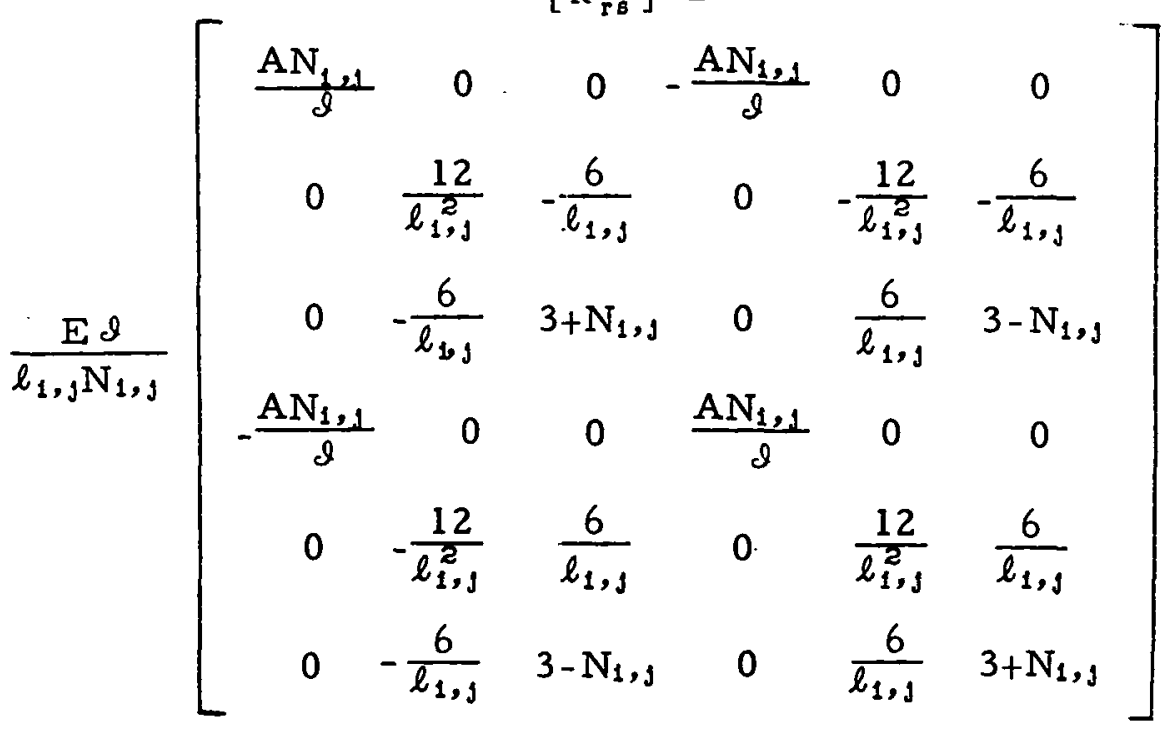

